MOLECULARLY IMPRINTING POLYMER SENSOR FOR THE DETECTION OF TESTOSTERONE AND ENDOCRINE DISRUPTING CHEMICALS IN ENVIRONMENTAL AND BIOMEDICAL APPLICATIONS

A Dissertation
presented to
the Faculty of the Graduate School
at the University of Missouri-Columbia
In Partial Fulfillment
of the Requirements for the Degree
Doctor of Philosophy

By

Abbas J. Kadhem

Dr. Maria Fidalgo, Dissertation supervisor

MAY 2021

CIVIL AND ENVIRONMENTAL ENGINEERING DEPARTMENT 
The undersigned, appointed by the dean of the Graduate School, have examined the

dissertation entitled

\section{MOLECULARLY IMPRINTING POLYMER SENSOR FOR THE DETECTION OF TESTOSTERONE ENDOCRINE DISRUPTING CHEMICALS IN ENVIRONMENTAL AND BIOMEDICAL APPLICATIONS}

presented by Abbas Jawad Kadhem,

a candidate for the degree of doctor of philosophy, and hereby certify that, in their opinion, it is worthy of acceptance.

Dr. Maria Fidalgo

Dr. Kathleen Trauth

Dr. Enos Inniss

Dr. Luis Polo-Parada

Dr. Chung-Ho Lin 


\section{ACKNOWLEDGMENTS}

I would like to express my gratitude to my supervisor, Dr. Maria Fidalgo for guiding my first steps as an independent researcher. Her patience, enthusiasm and great knowledge were highly appreciated.

I thank the four supervisory committee members, Drs. Kathleen Trauth, Enos Inniss, Luis Polo-Parada, and Chung-Ho Lin for reviewing a draft of my thesis, their questions, and notes that gave me during qualifying, comprehensive, and dissertation exams.

I really appreciate the support obtained from Dr. Chung-Ho Lin and his laboratory staff. The results described in chapter three of this thesis would not have been completed without their support. Many thanks to Dr. Luis Polo-Parada for helping me and great support he contributes to complete blood sensor project.

Assistance provided by my friends and college Mohammed Bayati, Mohamed Numman, Sally Qasim, Zahara Salahshoor, and Jingjing Dia, is appreciated. Also, Special thanks are extended to the Du'aa Kumayl group and Imam Hassan group for their support and encouragement.

My earnest thanks to the higher committee of education development in Iraq, for supporting and sponsoring to complete my studying in the US.

Words would never say how grateful I am to my wife for her continued and inexhaustible love, support and understanding during my pursuit of $\mathrm{Ph} . \mathrm{D}$ degree. I 
appreciate my kids, Murtaja, Misk, Mujtaba, Abulfadl and my little girl Mayar for enduring my ignorance and the patience they showed during my thesis writing.

My words standing powerless regard goes to my father and mother for their love and moral support. Without your supporting, inspiration, praying that you have given me, I think I might not be the person I am today. 


\section{TABLE OF CONTENTS}

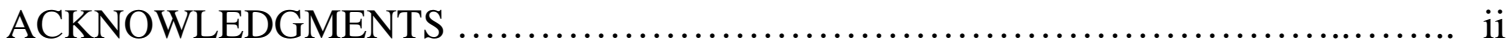

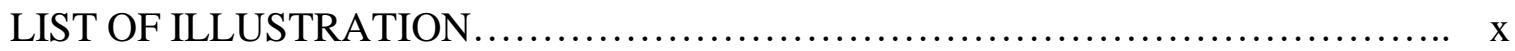

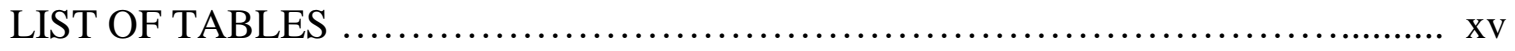

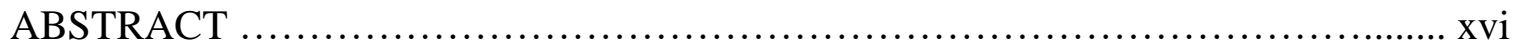

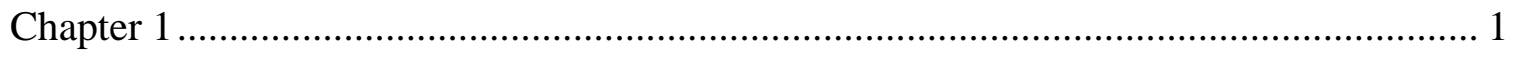

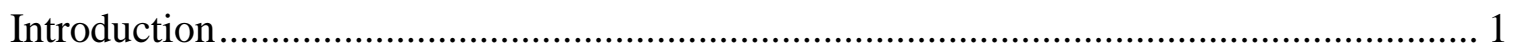

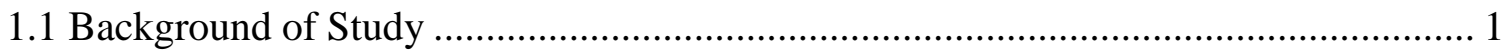

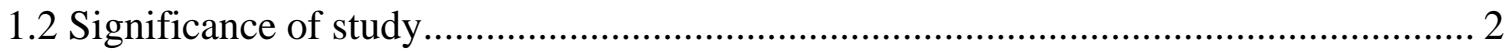

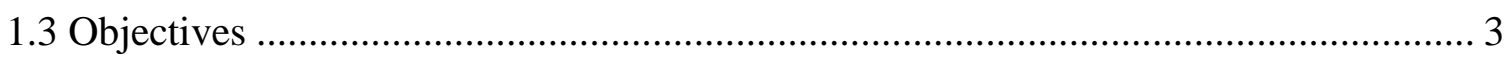

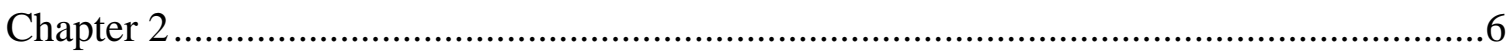

Molecular Imprinted Polymers (MIP) in sensors for environmental and biomedical

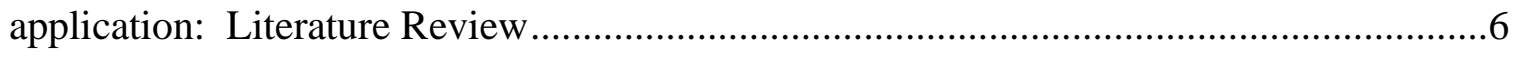

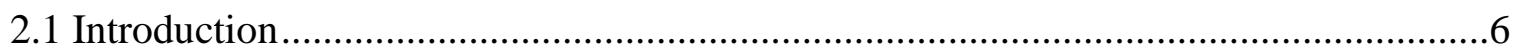

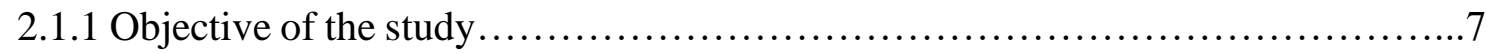

2.1.2 Typical Materials for MIP composition.................................................... 7

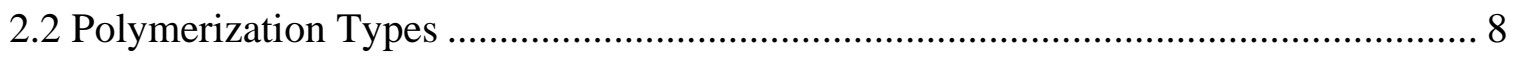

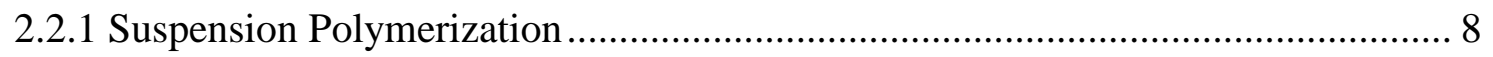

2.2.2 Precipitation Polymerization (dispersion polymerization) ............................... 8

2.2.3 Traditional Polymerization (bulk polymerization) ....................................... 9

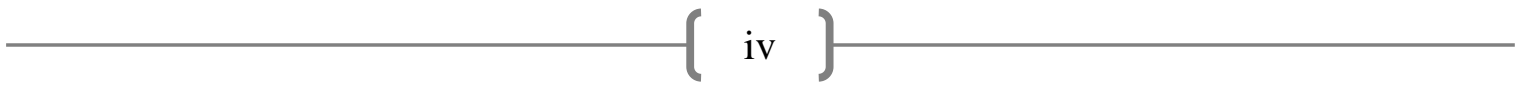




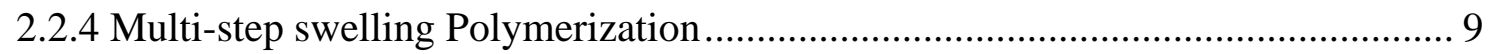

2.2.5 Emulsification Polymerization...................................................................... 10

2.2.6 Grafting Polymerization (surface polymerization) ………................................. 10

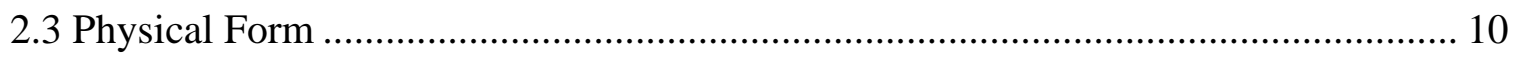

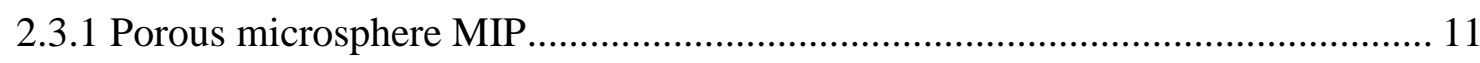

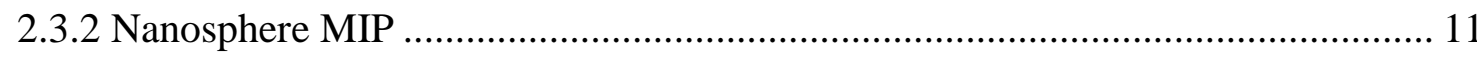

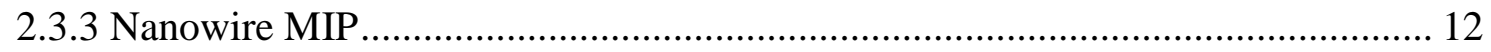

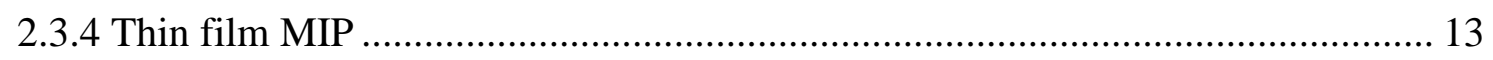

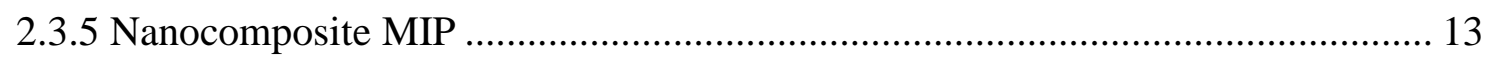

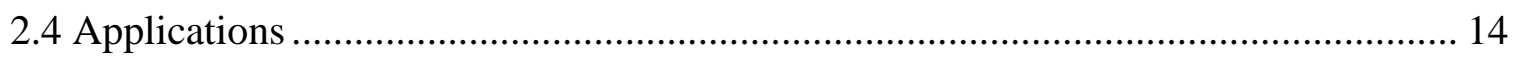

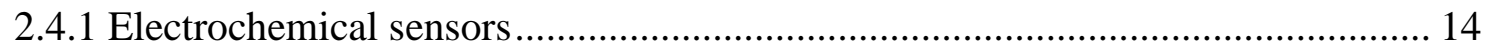

2.4.1.1 Environmental applications............................................. 16

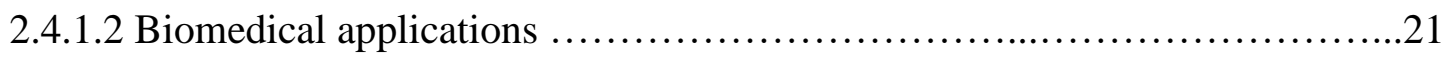

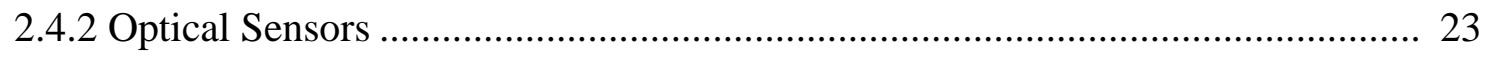

2.4.2.1 Environmental applications........................................... 24

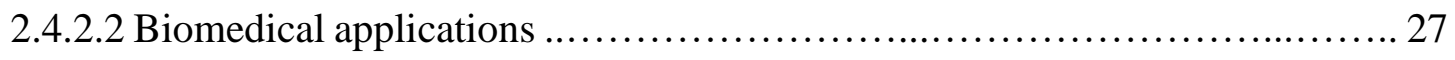

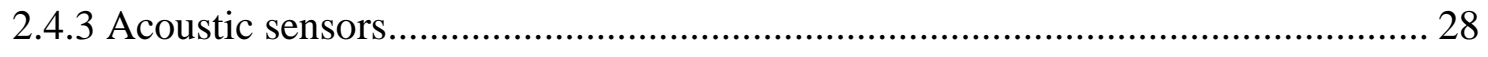

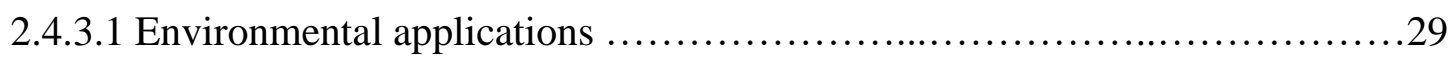

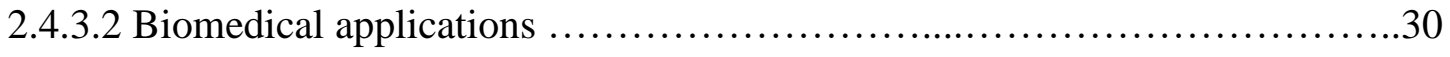


Chapter 3

Photonic Molecular Imprinted Polymer Film for the Detection of Testosterone in Aqueous Samples .33

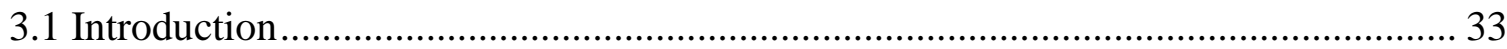

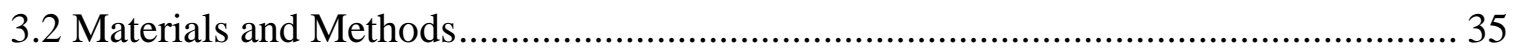

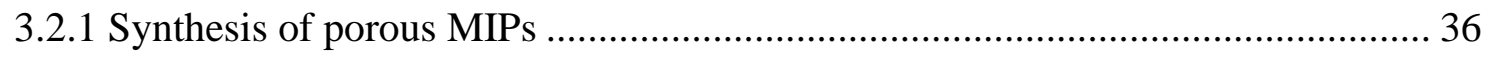

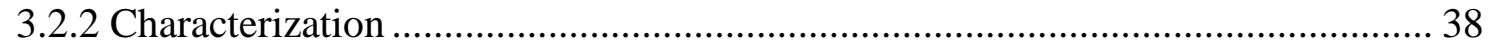

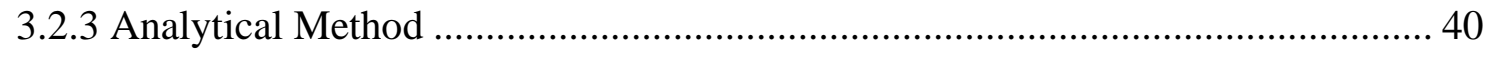

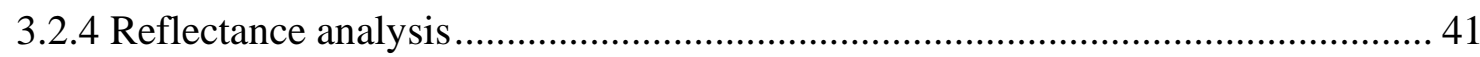

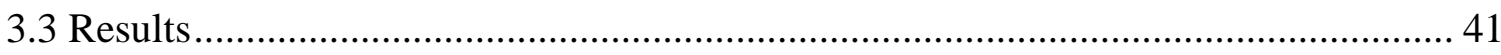

3.3.1. Characterization of silica particles and films .......................................... 41

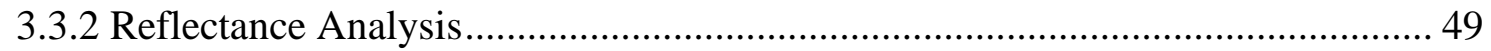

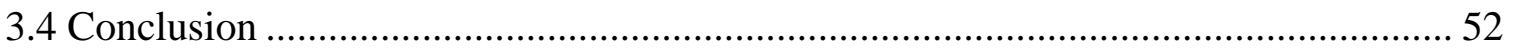

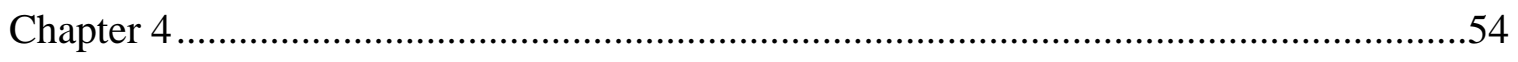

A novel Sensor to Detect of Testosterone(T) in Human Blood Based on Molecularly

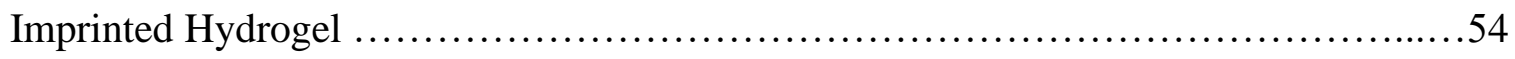

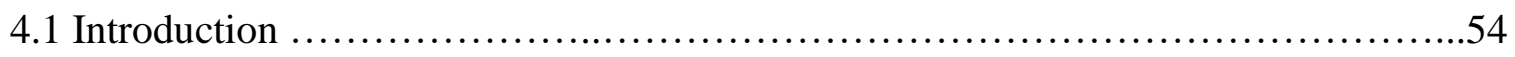

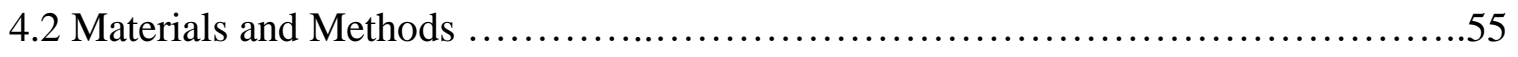

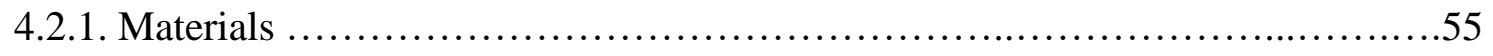

$($ vi $)$ 
4.2.2.1 Preparation of colloidal crystal slides .................................... 56

4.2.2.2 Synthesis of molecularly imprinted photonic hydrogel (MIPH) ..............57

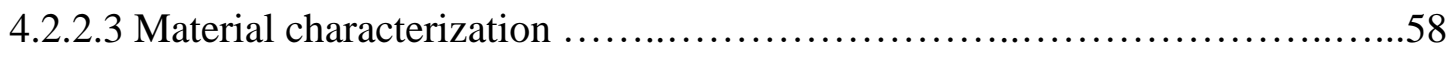

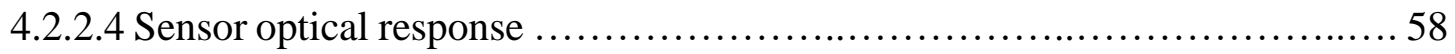

4.2.2.5 Analysis of interferences by structurally similar hormones .................. 59

4.2.2.6 Molecular modeling of interferences .....................................60

4.2.2.7. Analysis of blood samples .............................................61

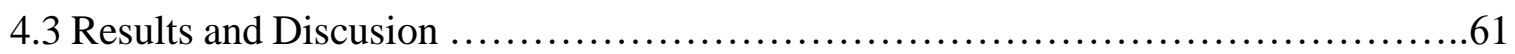

4.3.1 Characterization of silica particles and MIP …............................62

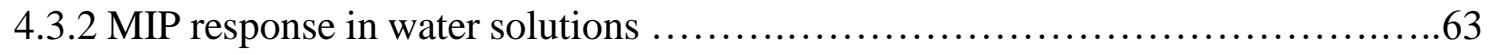

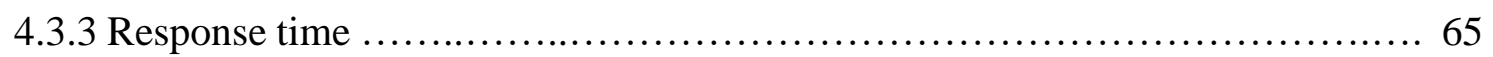

4.3.4 Investigation of possible interferences in blood samples........................66

4.3.5 Analysis of blood samples ................................................ 73

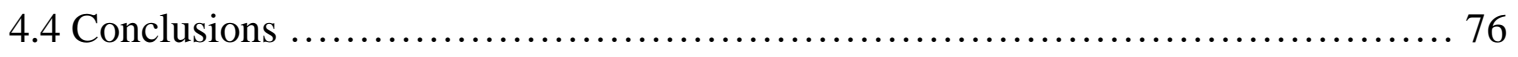

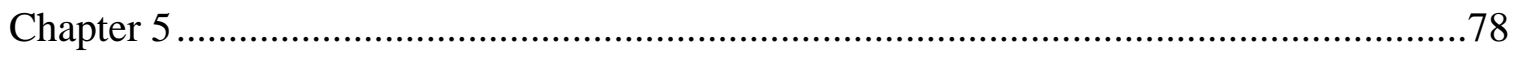

Using the Molecularly Imprinted Polymer Technique to Screen for Endocrine Disrupting

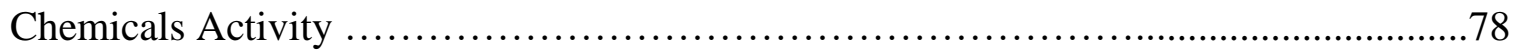

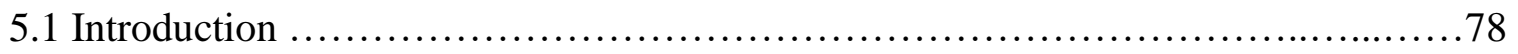

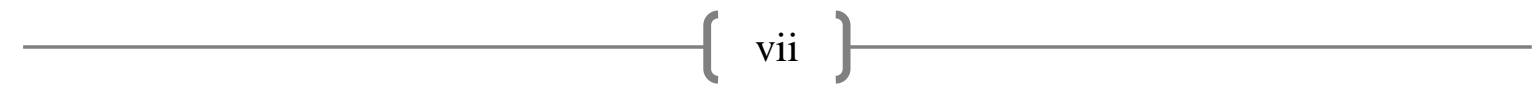


5.2.2.1 Preparation of silica particles colloidal crystals

5.2.2.2 Synthesis of molecularly imprinted photonic sensor

5.2.2.5 Selection of endocrine disrupting chemicals (EDCs)

5.2.2.7 The relation between the sensor response to testosterone and EDCs $(\lambda 1 \& \lambda 2)$ 


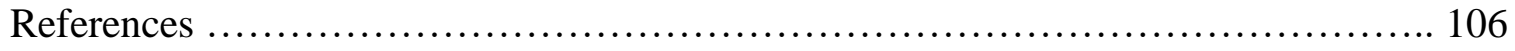

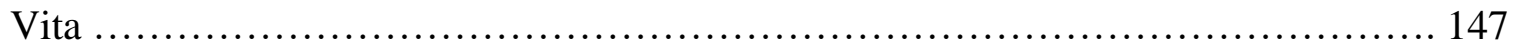




\section{LIST OF ILLUSTRATIONS}

FIGURE

PAGE

2.1 Number of MIP articles published each year since 1990 6

3.1 Schematic representation of the fabrication process and research design. 37

3.2 SEM images of: (a) colloidal crystal top layer; (b) colloidal crystal showing cross section; c) porous structure (top view) of the MIP s film; (d) internal morphology of the MIP film .43

3.3. Effect of $\mathrm{pH}$ on Swelling Ratio (\%) for polyacrylic acid films (shaded bars) and polymethacrylic acid films (clear bars)

3.4. FTIR spectra of the films at different synthesis stages: a) MIP after testosterone removal, b) NIP, c) MIP before testosterone removal. .46

3-5. Recognition Capacity for AA-MIP and AA-NIP at higher (a) and lower (b) range concentrations.

3.6. Kinetics of rebinding to unsupported MIP and NIP films, (a) initial testosterone concentration $5 \mathrm{ppm}$; (b) desorption of testosterone from MIP films initially incubated at

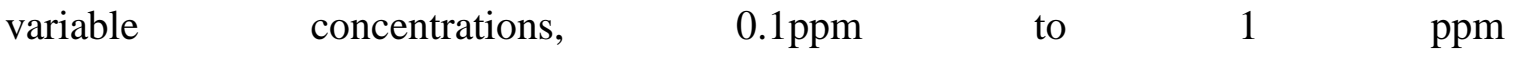
testosterone. .48 
3.7. Optical properties supported MIPs: (a) diffraction peak shift for MIPs and NIPs after incubation at various concentration of testosterone; (b) reflectance spectra of supported MIPs after incubation at various concentration of testosterone

3.8. Kinetic response of the testosterone MIP

3.9. Recoverability of photonic sensor for 6 cycles of use and Regeneration. .50

3.10. Optical response of the MIPs to testosterone and selected compounds. .51

4.1. Molecular structures of the $\mathrm{T}$ and interferences hormones; a) Testosterone b) dihydro testosterone c) dehydroepiandrosterone d) hydrocortisone e) dehydroepiandrosterone sulfate f) estradiol g) androstenedione

4.2. SEM image of (a) cross section of colloidal crystal, (b) Top view of silica particles colloidal crystal template (c) 2D structure of the MIP film. .62

4.3. Digital photo of the (a) silica colloidal crystal template, and (b) resulting MIP film.

4.4. Optical properties of sensors: (a) reflectance spectra of MIPs after incubation at various concentrations of T; (b) peak shift for MIPs after incubation at various concentration of T. .64

4.5. The differences in calibration curves for four MIPs

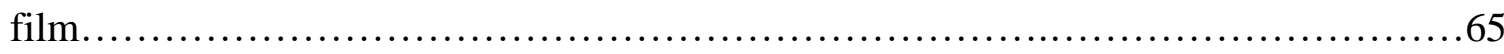

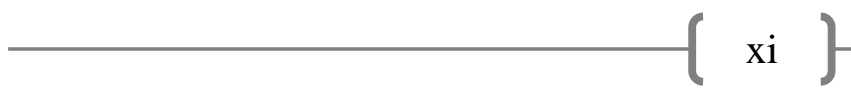


4.6. Kinetics of MIP response, (a) incubated in $1 \mathrm{ppb}$ of $\mathrm{T}$, (b) incubated in $5 \mathrm{ppb}$ of T. 66

4.7. MIP sensor response incubated in a $5 \mathrm{ppb} \mathrm{T}$ solution, in the presence of $0.04 \mathrm{ppb}$ of interfering hormones and $150 \mathrm{mM} \mathrm{NaCl}$, (a) wavelength shift (b) sensor $\mathrm{T}$ measured concentrations, calculated from wavelength shift and calibration curve. First bar corresponds to the control $5 \mathrm{ppb} \mathrm{T}$ solution in the absence of interferences. 69

4.8. MIP sensor response incubated in a $5 \mathrm{ppb} \mathrm{T}$ solution, in the presence of $5 \mathrm{ppb}$ of interfering hormones and $150 \mathrm{mM} \mathrm{NaCl}$, (a) wavelength shift (b) sensor $\mathrm{T}$ measured concentrations, calculated from wavelength shift and calibration curve. First bar corresponds to the control $5 \mathrm{ppb} \mathrm{T}$ solution in the absence of interferences.

4.9. MIP sensor response incubated in a $5 \mathrm{ppb} \mathrm{T}$ solution, in the presence of $30 \mathrm{ppb}$ of interfering hormones and $150 \mathrm{mM} \mathrm{NaCl}$, (a) wavelength shift (b) sensor $\mathrm{T}$ measured concentrations, calculated from wavelength shift and calibration curve. The first bar corresponds to the control $5 \mathrm{ppb} \mathrm{T}$ solution in the absence of interferences........................................................... 70

4.10. Top view of the molecular electrostatic potential surfaces (MESPs) for the interfering hormones. Regions with most negative and positive electrostatic surface potentials are colored with red and blue,

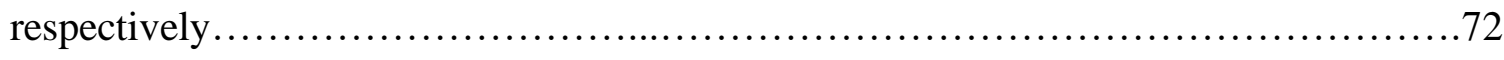

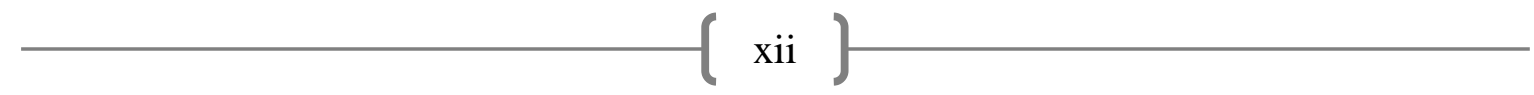


4.11. Side view of the molecular electrostatic potential surfaces (MESPs) for the interfering hormones. Regions with most negative and most positive electrostatic surface potentials are colored with red and blue,

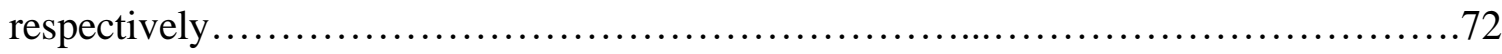

4.12. Application of sensors on a horse blood samples: (a) reflectance spectra of sensor after incubation at horse blood samples spiked with various concentrations of $\mathrm{T}$; (b) diffraction peak shift of sensor after incubation at horse blood samples spiked with various

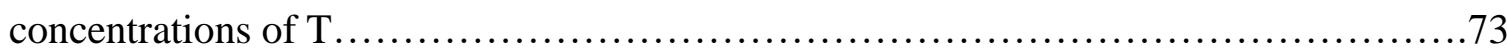

5.1. Synthesis of molecularly imprinted polymer and detection of EDCs .82

5.2 Structures of the Testosterone and eight of the endocrine disrupting chemicals 84

5.3. SEM images a) colloidal crystal of silica particles, b) nano-porous (top view) of MIP film. .91

5.4. Optical properties of sensors: (a) reflectance spectra of MIP's at various concentrations of T. (b) diffraction peak shift for MIPs after incubation at various concentrations of $\mathrm{T}$ .92

5.5. Toxicological prioritization index score for the eight endocrine disrupting chemicals (simulated data) for a model combining source data into 4 slices (AC50 with blue color, average lower concentration with red color, reference dose toxicity with gold color, and cancer potency value with green color). For each slice, the distance that the arc extends 
from the origin is proportional to its relative evidence of concern (e.g. longer $=$ greater risk), and the radial angle (width) indicates its weight in the overall model.

5.6. Peak wavelength shift for films incubated in solutions of variable concentration of testosterone and eight endocrine disrupting

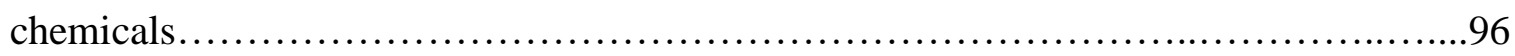

5.7. The relation between response of EDCs to the response of $\mathrm{T}$ with concentrations of these

chemicals

5.8. The binding affinity of testosterone as a comparison with the other EDCs at 1, 5, and $10 \mathrm{ppb}$ .98

5.9. The percent value for the EDCs concentrations as compare with testosterone concentration at the same wavelength shift at concentrations $5 \mathrm{ppb}$ and 10

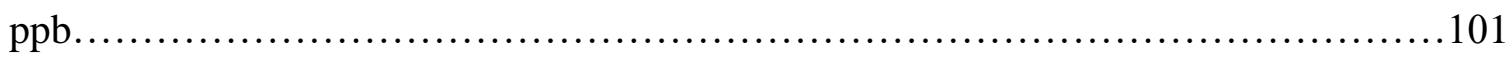




\section{LIST OF TABLES}

Tables

Pages

2.1. Summary of electrochemical sensor application

2.2. A summary of the technique was used by the optical sensor.

3.1. Recognition Capacity and Imprinting Efficiency of AA and MAA MIPs.

4.1. Interference of selected hormones on the assessment of a $5 \mathrm{ppb} \mathrm{T}$ solution by MIP sensor

4.2. $\mathrm{T}$ analysis in blood samples by photonic sensor and reference lab

5.1. The EDCs and their relevant data that was used in ToxPi software

5.2. Comparison of EDCs sensor affinity with RBA of Fang method. 


\title{
MOLECULARLY IMPRINTING POLYMER SENSOR FOR THE DETECTION OF TESTOSTERONE AND ENDOCRINE DISRUPTING CHEMICALS IN ENVIRONMENTAL AND BIOMEDICAL APPLICATIONS
}

\author{
Abbas J. Kadhem \\ Dr. Maria Fidalgo, Dissertation Supervisor
}

\begin{abstract}
Molecular imprinting is one of the promising techniques that have been used recently to detect trace contaminants in aqueous solution. This technique is based on the fact that the target compound is present during the polymer synthesis which gives an opportunity for the molecularly imprinted polymers (MIP) to rebind the target molecule selectivity after removal. In this thesis, it was used to detect a hormone (testosterone) in water and blood samples. The procedures are straightforward, fast, and use simple equipment. The detection of the template was carried out by using HPLC and UV-Vis. The MIP starts by preparing a template for the polymer morphology from a silica particle deposition on the glasses slides. At the beginning of this research, the silica particles were prepared by using the Stober method and then commercial silica particles were used. Bulk polymerization was used to prepare the polymer. Two types of solvent (porogen) have been applied. The composition of the prepolymerization solution was optimized. The smart sensor was used first as a self-standing film to characterize and validate. After that, the sensor was deposited on a Poly (methyl methacrylate) (PMMA) slide as a support material which made it easy to use and regenerate. The selectivity and sensitivity of the sensor to the target (testosterone) were studied. The sensor has the potential to detect testosterone
\end{abstract}


not only in a water sample but also in blood samples. In addition, this sensor has the potential for integration into a microdevice for on-site and online monitoring. Such a sensor could be easily used by an inexperienced operator. In this work, the sensor was developed to detect the target with a very low concentration in blood samples. Different endocrine disrupted chemicals were used to compete for the target and to test the potential interference effect. Several human blood samples were utilized to investigate the sensor selectivity. Also, the recoverability of the sensors was studied. The detection of endocrinedisrupting chemicals by traditional methods was complicated, expensive and timeconsuming. This research studied the affinity of eight EDCs to the testosterone sensor. In addition, the relation between the classification of chemicals depend on relative binding affinity (RBA) which calculated from other sources to the classification that were got from the sensor were compared to investigate any relationship between. Based on the results of the study, the chemicals were classified into 4 categories, according to their response: strong affinity (T), moderate (CHL, VIN, EST, and FLU), weak (BPA, DDE, and DCP), and inactive (DDT). Also, the percent activity showed that the selected chemicals had lower adsorption to the binding site of the sensor in comparison with testosterone. The results showed that $57 \%$ of our classification was identical with Fang classification which means that our sensor can be used as a pre method to study the affinity of EDCs binding to AR. 


\section{Chapter 1}

\section{Introduction}

\subsection{Background of Study}

Molecularly imprinting is the protocol of synthesis of an artificial receptor for the given target molecules based on the synthetic polymer. The molecularly imprinted polymer (MIP) synthesized contains target molecules or its derivative, one or two functional monomers, a crosslinker, a polymerization initiator, and a solvent [1]. Initially, the target molecules interact with the functional monomer to form a complex in an appropriate solvent, and the resulting complex is polymerized in the presence of a crosslinker. After polymerization, the template is removed to leave empty binding sites matching the template by size and shape [2].

The use of molecular imprinting in sensing of contaminants is a promising method due to the inexpensive and straightforward fabrication procedures. Optical sensors based on MIPs has received high attention recently as a result of development in optoelectronic devices and imaging techniques combined with low cost of light sources and detectors[3], [4]. Therefore, optical sensor technology is being applied in this work to the detection of endocrine disrupting chemicals and hormones.

Endocrine Disrupting Chemicals (EDCs) are a group of chemicals which are able to interfere with the endocrine system in numerous ways to produce undesired response or disruption, which may affect the health, growth, and reproduction of humans or other animals [5]. Some of these compounds are natural such as estrogen and androgen, and the others are industrial chemicals like the synthetic hormones, polyaromatic hydrocarbons 
(PAHs), polychlorobiphenyls (PCBs), pharmaceuticals and pesticides [6]-[8]. The presence of EDCs in the aquatic environment has concerned many scientists and regulators due to high resistance to the process of degradation and their ability to accumulate, adsorb to organic matter, and concentrate in biota [9], [10].

In this thesis, surface-contaminant interactions in porous structures were investigated. In particular an application in environmental engineering were explored: adsorption to engineered binding sites of a molecularly imprinted porous polymer, leading to change in the property of the polymeric film and its applications as a sensor.

The overall objectives of this dissertation is the detection of endocrine disrupting chemicals, specifically testosterone, at a very low concentration using an optical sensor in water and blood. The used technique is low cost, robust, straightforward, and rapid. The project was divided into several parts. The first part involved the fabrication of a thin film and the measurement of its ability to detect testosterone in water by validation with highperformance liquid chromatography (HPLC) analysis. Then, the film was supported on polymethyl methacrylate (PMMA) slides and combined with an inverse opal structure to fabricate a photonic sensor for the detection of testosterone. Third, the research focused on the detection of the hormone in blood samples. Finally, I explored the application of the testosterone molecularly imprinted sensor as a new tool to screen for activity of the endocrine disrupting chemicals using the portable sensor.

\subsection{Significance of study}

The molecularly imprinted polymer technique has significantly diversified, both from materials and instruments utilized, in the last decade in detecting and treating 
the contaminants. The numbers of technological applications are expected to continue increasing due to the development of approaches that used and demanded new and modern techniques. However, the instruments used for detection are expensive, complicated, and time-consuming. In this work, an optical sensor was developed to detect the EDCs by using UV-Vis spectroscopy in water first and blood in a second step.

In this study, we addressed some challenges and research gaps that were highlighted by previous research such as

1. The complicated, expensive, and time-consuming methods used to study the concentration of hormones.

2. The challenging, time-consuming, and expensive instruments required and the specific training to handle analytical equipment such as GC-MS, GC-MS/MS, or LC-MS/MS.

3. The difficulty and time-consuming of methods to detect EDs activity.

\subsection{Objectives}

The overarching theme of this dissertation is the application of the knowledge of organic molecule-surface interactions to solve environmental problems, specifically the capture and detection of hormones in different liquids. In order to address these concerns, the following three specific objectives were established

1) To fabricate a photonic sensor with the ability to detect testosterone and other endocrine disrupting chemicals in water .

2) To fabricate a photonic sensor with the ability to detect testosterone in blood. 
3) To fabricate a photonic sensor with the ability to screen for the EDCs activity in water, specifically on androgen receptor.

This document is divided into five chapters. After this first introductory chapter, a literature review of molecularly imprinted polymers is presented, with a focus on environmental and biomedical applications. The types of polymerization that were used before to prepare the MIPs are explained, as well as the physical forms in which the sensors were synthesized according to its applications and types of polymerization. Different types of sensors are described such as a porous microsphere, nanosphere, nanowire, thin-film, and nanocomposite. The biomedical and environmental applications for the different types of sensors are explored, such as an electrochemical, acoustic, and photonic sensor.

In the third chapter, a study of the molecularly imprinted polymer as a photonic sensor to detect the testosterone in the water is presented. In this section, the novel procedure combines the non-covalent imprinting method with the colloidal crystal template technique to produce a membrane with pre-specified morphology capable of selectively recognizing testosterone. The colloidal crystals made of silica particles were obtained by self-assembly techniques. After that, a polymerization solution was prepared, and two sandwich slides were made one of silica particles deposit, while the other is a PMMA slide to get the inverse opal on the surface of the plastic slide with cavities has the porosity with the same morphology of testosterone. The UV-visible was used to detect the template depending on the Bragg diffraction equation. When the analyte adsorption into the binding sites results in a change in Bragg diffraction of the polymer due to swelling or shrinking of the hydrogel film leading to a different peak for the adsorption, and therefore a clear optical signal can be detected. 
The fourth chapter presents the detection of testosterone in human and the horse blood samples. The sensor was prepared by colloidal crystal deposit first and then by inverse opal technique to get a homogenous 3D porosity with the same morphology of template. The research adjusted to use commercial silica particles deposit to make more homogenized each time with high reproducibility, then to develop the active inverse opal nanocavities. The sensor was prepared on the plastic strips (PMMA) and required short equilibrium time to detect the testosterone inside human and horse blood.

The last chapter reports the effectiveness of the testosterone MIPs to test ED activity of positive controls and selected agrochemicals. In this project, molecularly imprinted polymers were used to test the endocrine disrupting activity. This test can be performed by hormone reporter gene assays, but our proposed technique is more straightforward and faster. Several types of endocrine disrupting chemicals were used some of them have a similar structure to testosterone. The research was an effort leading to the understanding of the response of the sensor to different type of the hormones or EDC compounds to screen for ED activity in unknown samples. 


\section{Chapter two}

\section{Molecular Imprinted Polymers (MIP) in sensors for environmental and \\ biomedical application: Literature Review}

\subsection{Introduction}

Molecularly imprinting has been getting an increasing attention since first reported by Wulff in 1972 [11], [12], as shown in Figure (2.1), due to its simplicity, robustness, stability, and high selectivity [13], [14]. Molecularly imprinted is a tailor made polymer that depends on forming specific molecular recognition sites for a particulate target molecule [15]. The targeted analyte, is combined with the functional monomer to form a precursor by covalent [16]-[18], semi-covalent [19]-[21], or non-covalent [22]-[24] bonds and then polymerized in the presence of crosslinker and initiator. After that, the template is eluted by the suitable methodology to create the recognition cavities in the polymer matrix, with similar morphology to the template molecule [25], [26].

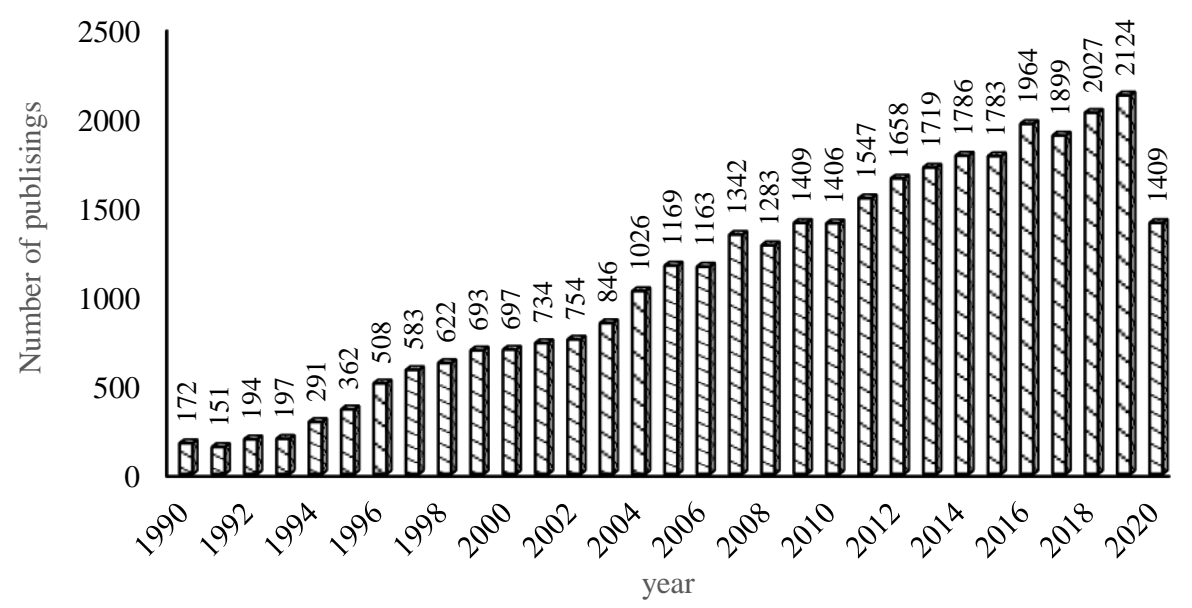

Figure 2.1 Number of MIP articles published each year since 2000. 


\subsubsection{Objective of the study}

Here we present a review of recent advances in imprinted molecular technologies applied in sensing for environmental and biomedical fields. Polymerization methods, physical forms, and materials are described. Electrochemical, optical, and acoustic sensor are reviewed, and the environmental and biomedical application of these sensors was studied.

\subsubsection{Typical Materials for MIP composition}

In the preparation of the molecularly imprinted polymers, the template and the monomer were incorporated to form a complex before polymerization. Then, the mixture was polymerized with the help of an initiator with a particular ratio of the crosslinker to form MIP. When the template was removed, 3D cavities were formed with the same morphology of template. The functional monomer has a significant effect on the physical properties and binding efficiency of the MIP[27] because of the binding interaction with the template resulting from the functional groups that found in his structure [28]. Different type of monomers were used to prepare MIPs. The most common monomers are methacrylic acid (MAA) [29], acrylic acid (AA) [30], acrylamide (AM) [31], 4vinylpyridine (4-VP) [32], 2-vinylpyridine (2-VP) [33], 2-hydroxyethyl methacrylate (HEMA) [34], methacryloylamidophenylboronic acid (MAPBA)[35] , 3-Aminopropyl triethoxysilane (APTES) [36], and phenyltrimethoxysilane (PTMS) [37]. In addition, numerous types of crosslinker, which is responsible for the structure stabilization of the MIP, were utilized such as ethylene glycol dimethacrylate (EGDMA) [38], divinylbenzene (DVB) [39], trimethylol-propane trimethacrylate (TRIM) [40], and N,Nethylenebisacrylamide (MBAA) [41]. Furthermore, the initiator is a necessary material to 
stimulate the polymerization [42]. There are different types of initiators used in preparing MIP's such as azobisisobutyronitrile (AIBN) [43], 1,1'-azobis(cyclohexane-1-carbonitrile) (ACC) [44], and 2,2-dimethoxy-2-phenylacetophenone (DPAP) [45].

\subsection{Polymerization Types}

Different types of polymerization have been reported in the preparation of molecularly imprinted polymers. These including: suspension polymerization, precipitation polymerization, bulk polymerization, multi-step swelling polymerization, emulsification polymerization, and grafting polymerization. Each one is explained in more details in the following section.

\subsubsection{Suspension Polymerization}

Suspension polymerization is heterogeneous polymerization containing two phases, liquid matrix, and monomer droplets, the monomer, and crosslinker are insoluble, so they make drops inside liquid matrix [46]. An adequate stabilizer is used to control the stability and the sizes of particles [47]. Different types have been applied such as surfactants [28], ionic liquids [48], and cetyl trimethyl ammonium chloride (CTAC) or vinyl benzyl trimethyl ammonium chloride (VBTAC) [49]. Solvents utilized are water [50], silicone oil [51], and polyvinyl alcohol [52], chosen for their compatibility with stabilizers.

\subsubsection{Precipitation Polymerization (dispersion polymerization)}

Precipitation polymerization has many advantages, as it does not require any mechanicals operation after polymerization such as grinder, sieving, and sedimentation. Also, it does not need to use a surfactant or any stabilizer which may lead to contaminant 
the MIP. In addition, uniformly sized spherical particles are formed [15].Ye and coworkers [53] were the first authors to use this technique. In this type of polymerization, monomer, initiator, and crosslinker form the polymer particles and precipitate out of the homogenous solvent as a result of the low solubility of the polymer [54]. Molecularly imprinted polymers of different shapes can be synthesized by precipitation polymerization such as a microsphere [55], nanosphere [56], core-shell [57], thin film [58], and nanocomposite [59].

\subsubsection{Traditional Polymerization (bulk polymerization)}

Traditional polymerization is the most comprehensive used method which involves the mixing of the monomer, the crosslinker, initiator, and the template in a mold polymerization and then grinding and sieving after removal of template [60]. Despite this method being easy and straightforward, it has some drawbacks such as slow rebinding kinetics [61], potential incomplete elimination of template molecule, irregular particle shapes, and the inhomogeneous allocation of the binding sites [62].

\subsubsection{Multi-step swelling Polymerization}

Multi-step swelling polymerization is another type of polymerization, which involves the suspension of uniform seeds inside water and the addition of solvent to a batch with stirring for a specific time until the seeds start to swell and become particles. After that, the particles will polymerize after one step of swelling or more [63]. The morphology of the result particles is affected by the cross-linker density of the seeds, the temperature, and the ratio of monomer/seed [64]. 


\subsubsection{Emulsification Polymerization}

Emulsification Polymerization is resulting from the reaction between free radical polymerization and a hydrophobic monomer inside the heterogeneous aqueous system. The reaction initiated by water or oil soluble free radical initiator. The dispersion polymer'latex" is milky. The particles size resulting from this method is between $10 \mathrm{~nm}$ and $1000 \mathrm{~nm}[65]$, [66].

\subsubsection{Grafting Polymerization (surface polymerization)}

Grafting Polymerization involves a thin film polymer grafted on the surface of chromatograph-grade porous silica. Several advantages were found by using this technique such as high surface density of polymer chains, high stability of coated layer, and the ability to graft several polymers to the same substrate [67], [68].

\subsection{Physical Form}

The molecularly imprinted polymer can be synthesized in different physical forms such as a porous microsphere, nanosphere, nanowire, thin film, and nanocomposite according to its application and polymerization method [69]. The various types of MIP are necessary because it can be used in the advance application, and can overcome the disadvantages that found in the earlier form of MIP, monoliths, which was prepared by bulk polymerization and then sieved and grind to obtain particles. This method was time consuming, less efficient and yielded particles of irregular size. Therefore, researchers tried to develop several forms according to the type of template and the needs of the application. 


\subsubsection{Porous microsphere MIP}

As opposed to particles prepared by traditional polymerization methods, the porous microspheres have both internal and surface porosity, which results in desirable properties such as high surface area, low density, and a high capacity [46]. The porous microsphere MIPs can be prepared by different types of polymerization such as suspension polymerization [50]-[52], [70]-[73], precipitation polymerization [74]-[77], emulsification polymerization [78], grafting polymerization [79], step swelling polymerization [80], and sometimes, it is a combination of two methods [81]. Bougrini and co-workers [82] prepared MIPs based on a microporous-metal-organic framework (MMOF) film on a gold electrode for detection of tetracycline (TC). The MMOF was resulting from the deposit of aniline moieties of P-amino-thiophenol functionalized $\mathrm{Au}-$ nanoparticles by electropolymerization in the presence of TC as a template molecule. The template was extracted by immersing the electrode in a solution of phosphate buffer, $\mathrm{pH}=7.4$, and methanol $(1: 1(\mathrm{v} / \mathrm{v}))$

\subsubsection{Nanosphere MIP}

The fabrication of MIP nanoparticles produces high surface area to the volume ratio. Therefore, easily created, inexpensive, and can lead to high recognition of different chemicals applied in a variety of fields [12]. Chen and coworkers [83] prepared molecular imprinted hollow Nanospheres (MIHSs) to detect the hemoglobin. The size of particles was $360 \mathrm{~nm}$ and polymerized by using grafting polymerization. Stöber method [84] was used to synthesize the silica particles and modified by 3-glycidoxypropyltrimethoxysilane (GLYMO). After polymerization, hydrofluoric acid and formic acid were sequentially used to etch the $\mathrm{SiO}_{2}$ and remove the template. In another approach, nanospheric molecularly 
imprinted polymer NMIP was used as a piezoelectric sensor to detect antibodies [85]. The NMIP was synthesized by precipitation polymerization. Template, initiator with different monomers (MMA, MAA, and 4-VP) and crosslinkers ((EGDMA, TRIM, and DVB), were applied to discover the effective mixture for the NMIP. A thermostatic water bath was used to make the polymerization with constant stirring for 3-4 hours at the temperature of 40-60 ${ }^{\circ} \mathrm{C}$. The template was removed by applying acetonitrile in Soxhlet extractors for 6 hours.

\subsubsection{Nanowire MIP}

The nanowire MIP sensor is a metallic or semiconductor particle with a diameter of $<1 \mu \mathrm{m}$ and length of tens of micron [86]. This approach increases the availability of binding sites on the surface of MIPs due to the high surface area to volume ratio [87]. López et al. [88] prepared an optical sensor using a silica-silver nanowire (AgNWs) to detect the tryptophan (Trp). The Trp molecular imprinted silica coated AgNWs to get the Trp-MIP-SiO 2 -AgNWs. Sometimes non-imprinted nanowire substances were used with the MIP as reinforcement materials. Chen and et al. [89] prepared a thin film of proteinimprinted polymer coating the silicon nanowire (SiNWs) as a strengthening material. The low density and high hydrophobicity of SiNWs made it suitable to synthesize the MIPs. The MIPs were prepared by dispersion of SiNWs in Tris buffer by ultrasonication and mechanical stirring. The template, bovine hemoglobin $(\mathrm{BHb})$, was added to the starrier solution. After $1 \mathrm{hr}$ the monomer, dopamine, was added, and the polymerization process was continued for $3 \mathrm{hr}$. Finally, the template was extracted by a mixture of hydrochloric acid $(1 \mathrm{~mol} / \mathrm{L})$ and methanol $(1: 4 \mathrm{v} / \mathrm{v})$. The BHb-MIP exhibited the nanowire morphology under Scanning Electronic Microscopy (SEM), and Transmission Electron Microscopy (TEM). 


\subsubsection{Thin film MIP}

Thin film molecularly imprinted polymers can be prepared by direct polymerization of imprinting mixture on the supporting materials or membrane support called molecularly imprinted polymer membranes [28]. Boysen and coworkers [90] synthesized double layer films onto a silicon wafer. The first thin film was deposited on the silicon wafer by spincoating and photopolymerized under UV light at a wavelength of $365 \mathrm{~nm}$. The monomer MAA was mixed with the template N-boc-L-phenylalanine, TRIM as crosslinker, and DPAP as an initiator in different amounts in a diglyme as a solvent. After polymerization, a thin film of MAA-MIP was formed on the surface of the silicon wafer, and the template was removed by washing the film with a methanol/acetic acid (v/v 90:10) solution. Then, another pre- polymerization solution containing $4 \mathrm{VP}$ as a monomer, and the same other chemicals were spin coated on the first thin film MIP with the placement of a gold grid. Finally, after polymerization, the grid was removed to form a grid pattern on the surface of the film. Egli and coworkers [91] prepared a thin film molecularly imprinted polymer with a quasi-template to detect the PAHs. The thin film was prepared by mixing of the pseudo template (toluene), EGDMA as crosslinker, 4-VP as a monomer, DMPA as an initiator, and 1-octanol as a porogen. The solution was polymerized under UV light, and the template was removed using ethyl ether.

\subsubsection{Nanocomposite MIP}

Different types of nanocomposite MIPs were used to improve the efficiency of the MIPs to detect the contaminants. One of the practical ways to obtain a nanocomposite MIP was done by combining Fe3O4 with Graphene (G)/ Graphene oxide (GO). The $\mathrm{Fe}_{3} \mathrm{O}_{4} / \mathrm{G} / \mathrm{GO}$ nanocomposite was used due to the separation ability of the $\mathrm{Fe} 3 \mathrm{O} 4$ 
nanoparticles and adsorption capability of the G/GO to the target molecule from the mixture through imprinted polymer [26]. Arabzadeh and co.workers [92] developed a nanocomposite sensor composed of nano photocatalyst of $\mathrm{TiO}_{2}$, and nanomolecular imprinted polymer for enhanced photodegradation of tartrazine. Precipitation polymerization was used to synthesize the nanoMIP with nano-photocatalyst. They utilized acrylamide as functional monomer, $\mathrm{N}, \mathrm{N}^{\prime}$-methylene-bis-acrylamide as cross-linking agent, tartrazine as a template, and $\mathrm{TiO}_{2}$ as a nano-photocatalyst.

\subsection{Applications}

Molecularly imprinted polymers can be used in different applications such as separation [93], [94], adsorption [95], [96], catalysis [97]-[99], solid phase extraction [100]-[104], drug delivery [105]-[107], membrane filtration [108]-[112], and sensing. The latter is the focus of this review.

\subsubsection{Electrochemical sensors}

The electrochemical sensor consists of an electrochemical cell, which contains two or three electrodes. The electrochemical sensor can be classified into three types, depending on the electrical parameter being measured: conductivity/ capacitance electrochemical sensors which measure the variety in conductance over time, a potentiometric sensors which measure the modification in membrane potential, and voltammetric/ amperometric sensor which measure the various in current throughout the electrochemical response where the applied voltage is measured [113], [114].Table 2.1 summarizes recent reports of MIP based electrochemical sensors 
Table 2.1. Summary of electrochemical sensor applications.

\begin{tabular}{|c|c|c|c|c|}
\hline $\begin{array}{l}\text { Transduction } \\
\text { scheme }\end{array}$ & MIP types & Analytes & $\begin{array}{l}\text { Linearity } \\
\text { /Detection } \\
\text { Limit } \\
\end{array}$ & Reference \\
\hline \multirow{4}{*}{ Capacitance } & Apta-MIP & prostate specific antigen & $3.67 \times 10^{-12} \mathrm{M}$ & {$[115]$} \\
\hline & MIP & $\mathrm{N}$-formylamphetamine & $10^{-5} \mathrm{M}$ & [116] \\
\hline & GR-MIP/GCE & L-5-hydroxytryptophan & $6 \times 10^{-9} \mathrm{M}$ & [117] \\
\hline & MIP/sol-gel technique & $\begin{array}{l}\text { methidathion } \\
\text { organophosphorous } \\
\text { insecticides }\end{array}$ & $1.7 \times 10^{-5} \mathrm{M}$ & [118] \\
\hline \multirow{3}{*}{ Potentiometric } & MIP-based sensor & propranolol & $10^{-5}-10^{-1} \mathrm{M}$ & [119] \\
\hline & $\begin{array}{c}\text { GP/GNSs/LOS-MIP/(BMP)Tf }{ }_{2} \mathrm{~N}- \\
\text { CPE }\end{array}$ & Losartan & $1.82 \times 10^{-9} \mathrm{M}$ & {$[120]$} \\
\hline & MWCNT-MIP & Lindane & $1 \times 10^{-10} \mathrm{M}$ & [121] \\
\hline \multirow{15}{*}{ Voltammetric } & MIPMs & glucose & $0.01 \mathrm{M}$ & [122] \\
\hline & $\begin{array}{l}\text { MIP-SAM electrode (self- } \\
\text { assembled membrane) }\end{array}$ & Lead (II) & $2 \times 10^{-7} \mathrm{M}$ & {$[123]$} \\
\hline & TATP-MIP & Triperoxide & $1.8 \times 10^{-7} \mathrm{M}$ & {$[124]$} \\
\hline & CNT-IL/MIP & melamine & $1.1 \times 10^{-7} \mathrm{M}$ & {$[125]$} \\
\hline & MIP/rGO@Au/GCE & carbofuran & $2 \times 10^{-8} \quad \mathrm{M}$ & {$[126]$} \\
\hline & MIP-MWNTs-IL@PtNPs/GCE & tartrazine & $8 \times 10^{-9} \quad \mathrm{M}$ & {$[127]$} \\
\hline & MIP/MWCNT/GCE & ochratoxin A & $4.1 \times 10^{-9} \mathrm{M}$ & [128] \\
\hline & MIP/MWCNTs/PGE & Triamterene & $3.35 \times 10^{-9} \mathrm{M}$ & [129] \\
\hline & MIP/ATO-sol-gel/CS/Pt & $\beta_{2}$-Agonists & $1.7 \times 10^{-9} \mathrm{M}$ & [130] \\
\hline & MIP/MWCNT/GCE & valganciclovir & $0.3 \times 10^{-9} \mathrm{M}$ & {$[131]$} \\
\hline & AuNPs@MIP/CKM-3/P-r-GO/GCE & dimetridazole & $5 \times 10^{-10} \mathrm{M}$ & {$[132]$} \\
\hline & MIP/NPAMR & dopamine & $7.63 \times 10^{-14} \mathrm{M}$ & {$[133]$} \\
\hline & AuNPs@MIP & dopamine & $7.8 \times 10^{-9} \mathrm{M}$ & [134] \\
\hline & TAM-MIP & Tamoxifen & $1-100 \times 10^{-9} \mathrm{M}$ & {$[135]$} \\
\hline & NF-MIP & norfloxacin & $1 \times 10^{-10} \mathrm{M}$ & [136] \\
\hline
\end{tabular}




\subsubsection{Environmental applications}

Alizadeh et.al successfully designed a voltammetry electrochemical sensor for 2,4,6-trinitrotoluene (TNT) determination in natural water and soil samples. The sensor method was cheap and straightforward. The TNT-MIP particles were prepared by blending the template, methacrylic acid (MAA), and dry chloroform into the volumetric flask. Then, EDMA and AIBN were appended to the mixture. After ejecting the oxygen by Nitrogen, the polymerization was done by heating in a water bath for $24 \mathrm{hr}$ at $60^{\circ} \mathrm{c}$. The polymer was powdered, and the template was removed. Subsequently, in order to form a sensor (MIP$\mathrm{CP}$ or NIP-CP), the MIP or NIP particles were blended with graphite, in the presence of neicosane. A carbon paste electrode was used to increase the selectivity of the MIP because of increasing of electrode response. The increased response in the electrode is resulting from the electron transfer which was capable of enhancement the electrode in the presence of higher carbone paste. The structure of the MIP-CP electrode such as MIP, carbon, and n-eicosane was studied to find the optimum composition of the electrode. Also, the effect of washing electrode was investigated which showed that washing had high effected on the selectivity of the electrode because it removed the weakly absorbed interference from the electrode which can be utilized for selectivity improvement and omit the interference effect [137]. The prepared sensor showed a linear relationship over TNT concentration in the range $5 \times 10^{-9}$ to $5 \times 10^{-6} \mathrm{M}$ with a detection limit of $1.5 \times 10^{-9} \mathrm{M}$. On the other hand, the main problem of this sensor that it worked just in acid phase $(\mathrm{pH}=4.5)$ which may make it difficult to use in the environment.

In another work, the same team [138] used the corresponding electrode for detection of para-nitrophenol. Graphite as a powder was mixed in a mortar with the para- 
nitrophenol MIP or NIP in a different amount for $10 \mathrm{~min}$. After that, the mixture was added to melted n-eicosane and mixed. Finally, the paste was used to fill the holes of the electrode body, which were removed after cooling. Several conditions were studied, and their effect on the para-nitrophenol extraction in the electrode. Different range of $\mathrm{pH}(3-6)$ were investigated, and the optimum $\mathrm{pH}$ was 5 . The effect of pre-potential amount and exertion time were discussed and found that the optimum amount and time of pre-potential were $1.0 \mathrm{~V}$ and $25 \mathrm{~s}$, respectively. The sensor was used in different real water samples with a low concentration of para-nitrophenol and compared with similar works. The linear relationship was between $8 \times 10^{-9}$ to $5 \times 10^{-6} \mathrm{M}$ and the detection limit $3 \times 10^{-9} \mathrm{M}$. However, the authors did not study the effect of analogs on the selectivity of the sensors.

Neto et al. investigated an approach to detect 4-aminophenol (4-Aph) by using hemin-based MIP as an amperometric electrode. The MIP film was synthesized by precipitation polymerization of a solution compound of MAA, trimethylolpropane trimethacrylate (TRIM), AIBN, hemin dissolved in a mixture of dimethylsulfoxide (DMSO), acetonitrile solution, and template. Later, the hemin-MIP particles, suspended in ethanol, were mixed with nafion in glassy carbon electrode surface and dried at room temperature. The hemin -based MIP sensor was tested in tap and river water samples. The recovery values obtained were in the range of $93-111 \%$ which indicated that the adapted electrode was a highly selective amperometric sensor for 4-Aph detection [139]. Also, the linearity and the detection limit were $10^{-5}-9 \times 10^{-5} \mathrm{M}, 3 \times 10^{-6} \mathrm{M}$. One limitation of this study is that the authors neglected to consider the effect of interferences in water such as dissolved solid and organic matter. 
In his study for determination of 2-nonylphenol (2-NP) in water and soil samples, Zhang and et al. [140] prepared a molecularly imprinted polymer based on sol-gel and multiwall carbon nanotube-nafion. Nafion increased the homogeneity of MIP sol-gel and improve the peak current of the electrochemical probe. Three type of monomers were used (APTES, PTMS, and combined of APTES and PTMS). The combined APTES and PTMS polymer produced high discriminating voids and strong interaction forces e.g. hydrogen bond. The kind of monomer, $\mathrm{pH}$, and incubation time were studied. The optimal value of $\mathrm{pH}$, which affected the degree of ionization of 2-NP and electrostatic binding of 2-NP in MIP, was 7. The stability and repeatability were calculated by differential pulse voltammetry (DPV) and showed a RSD for the sensor of $3.6 \%$ for 20 successive measurements. Also, the MIP had a higher current response to the 2-NP compared to structure analogs. The study showed that the sensor can be applied efficiently to detect 2$\mathrm{NP}$ with linearity in the range from $2 \times 10^{-7}-36 \times 10^{-6} \mathrm{M}$, whereas, the detection limit was $0.6 \times 10^{-7}$. It should be mentioned that the article did not study the effect of physical or chemical interaction in real samples such as those that can arise due to the presence of suspended solids.

Moreover, Thayyath et al. [121] reported a new and convenient synthetic procedure to obtain a molecularly imprinted polymer- based potentiometric sensor for determination of lindane $(\Upsilon-\mathrm{HCCH})$, an organochlorine pesticide, by using multi-walled carbon nanotubes (MWCNT). The potentiometric sensor was prepared using a Cu-electrode as an active electrode and the calomel electrode as the reference electrode. The MWCNTs were mixed with glycidyl methacrylate (GMA) because GMA contains epoxide groups which essential in developing the polarity of MWCNT. Many characteristics were studied pH, 
sensitivity and detection limit, reusability, and selectivity with different types of samples tap water, wastewater, and ground water. The optimal value of $\mathrm{pH}$ was found to be 3 , where at this point the negative charge of chlorine atoms found in $(\Upsilon-\mathrm{HCCH})$ and protonation of a (-COOH) group of the MWCNT interact by electrostatic force. This interaction affected the sensitivity of the MIP. In addition, reusability of the MIP was studied, and it was found that it could be used efficiently until nine times with a maximum loss of efficiency about $0.5 \%$ in the first three trials, after which, the efficiency remained constant. The MIP showed high selectivity as a compared to the other pesticides. The sensor was not adjusted to work with different $\mathrm{pH}$.

Another successful electrochemical sensor was the molecularly imprinted polymer modified boron-doped diamond (BDD) electrode (MIP-BDD) for selective resolution of sulfamethoxazole (SMX) in water. Three electrodes were used a working electrode (MIPBDD), and a platinum and a calomel electrode for control. In addition, four factors ( $\mathrm{pH}$, template concentration (Csmx), electro-polymerization factor, and the monomer concentration (Cpy)) were studied. The optimum $\mathrm{pH}$ was 7 , where the response current $(\Delta \mathrm{I})$, a blank current (Iblank) minus peak current (Ipeak), was almost twice that at $\mathrm{pH}=6$ and 9. Also, the LOD was found to be $24.1 \times 10^{-9} \mathrm{M}$ which was much lower than in the other studies. The reliability of the sensor was measured by experiments with $\mathrm{RSD}=2.31$ for the $(\Delta \mathrm{I})$ and after 30 days of keeping the sensor in glass desiccator under room temperature the value of $97.7 \%$ of the primary value. To assess the selectivity of the sensor, the author used three chemicals sulfadimethoxine (SDM), sulfadoxine (SD), and sulfadiazine (SIZ) with very similar structure to that of SMX. The sensor showed good selectivity for the SMX. Finally, the sensor exhibited an excellent recovery between 96- 
$106.2 \%$ for the detection of SMX in water samples [141]. However, since the concentration level of SMX in real water is usually much lower than the detection limit, the sensor would needs to be improved to be used in water and wastewater samples.

A hybrid electrochemical sensor has been developed for the detection of $\beta$-Estradiol (E2) in water based on molecularly imprinted polymers [142]. The sensor was composed of EGDMA as crosslinker monomer, acetonitrile as a porogen, and MAA was used as the functional monomer. Three electrodes were used. The working electrode was an Au, whereas the reference and counter electrode were from $\mathrm{Ag} / \mathrm{AgCl}$ and a Pt respectively. Computational molecular modeling was applied to test the best monomer/template ratio [143], [144].The detection limit of the sensor was $6.89 \times 10^{-7}$. To verify the selectivity of estradiol-MIP, three compounds were used 17 $\alpha$-estradiol ( $\alpha$-E2), estriol (E3), and progesterone (P4). The sensor showed high selectivity for E2 at different concentration when it was a test with ( $\alpha$-E2) and (P4), but it lost some selectivity when E3 was present at high concentration. The authors explained that the sensor had a selectivity to the E3 in addition to E2 because of the similar structure between E2 and E3, where the difference is only an additional hydroxyl group in E3.

A MIP-QCM (quartz crystal microbalance) was devised for determination the atrazine (ATR) in waste water sample[145]. For this chemosensor, the MIP film was prepared by mixing the atrazine with phenol monomer in the presence of phosphate buffer. After that, AIBN was added as an initiator, 2-hydroxyethyl methacrylate (HEMA) and EGDMA under a nitrogen flow as a monomer crosslinker. Then, the complex was added to the QCM chip to form a thin film on the surface. Finally, the surface QCM chip was polymerized under UV light. The determination of ATR in wastewater by QCM and LC- 
MS/Ms showed that there was no major different between them. Also, the detection limit was $28 \times 10^{-12} \mathrm{M}$ which represents a very high sensitivity to the ATR. In addition to the sensitivity, the selectivity of the developed sensor appeared that the imprinted QCM chip had more selectivity toward the ATR than the simazine (SIM) and prometryn (PRO). Despite these good result, the main problem is the instruments that used to detect the atrazine, LC-MS/MS or QCM, was so expensive. So, the authors need to devise sensors were able to detect herbicides by cheap methods.

\subsubsection{Biomedical applications}

Da Silva, and co-workers [146] prepared electrochemical sensor consisting of a multi - wall carbon nanotube (MWCNTs) deposited on the surface of the glassy carbon electrode, coated with a molecularly imprinted polymer film via cyclic voltammetry. The MWCNTs were used to give stability, high electric conductivity, a high surface area, which enhanced the sensitivity and selectivity of the sensor. Also, polypyrrole was feasible in the production of molecularly imprinted electrochemical because of ability to immobilize target compound. The MIP/MWCNT/GCE was used to detect norfloxacin (NFX), an antibacterial agent, in human urine. The sensor showed linear behavior between $1 \times 10^{-7}$ and $8 \times 10^{-6} \mathrm{M}$, and the detection limit was $46 \times 10^{-9} \mathrm{M}$. The sensor recognized with high sensitivity the target when it was present with four different analogs: urea, ascorbic acid, glucose, and enrofloxacin. Reusability reached to 30 cycle of use without major change to the current response signal. However, according to the interference was found between the NFX and enrofloxacin, the sensor needed improvements in its selectivity.

MIPs with recognition sites for imidocarb dipropionate, a veterinary drug, were 
synthesized based on potentiometric sensor [147].The potentiometric sensor reading depended on the difference in potential between membrane sensor electrode and reference electrode $\mathrm{Ag} / \mathrm{AgCl}$. The membrane sensor was fabricated by sonication the mixture of the template, MAA, EGDMA, benzoyl peroxide, and acetonitrile. Then, the mixture was polymerized by using bulk polymerization. After that, the MIP particles were mixed with PVC powder. Finally, the combination was poured into a glass cup and left to dry at room temperature. Several optimization parameters were studied $\mathrm{pH}$, type of plasticizer, stability, and selectivity of the sensor. $\mathrm{pH}=7$ was found to be the optimum $\mathrm{pH}$. The response time, the time required to achieve a steady-state potential was $10 \mathrm{~min}$. The MIP-based sensor was successfully used to detect the imidocarb dipropionate in liver and kidney tissue of bovine animals with a limit detection of $2 \times 10^{-6} \mathrm{M}$, and the response linearity reached to $10^{-5}-10^{-2}$ at response time equal to $10 \mathrm{~min}$. The sensor showed an excellent exposure to the imidocarb dipropionate in the liver and kidney of bovine.

A novel potentiometric sensor was designed by molecularly imprinted polymer on the glassy carbon to detect heparin in heparin sodium injection [148]. The heparin imprinted polymer was prepared by adding heparin as a template, MAA as functional monomer, AIBN as an initiator, EGDMA as a crosslinker to the benzene. The prepared glassy carbon was rinsed in the prepared solution for 2 mins, and then the process was repeating until a thick film was formed. After that, the thick film coated electrode was polymerized under UV- lamp. Then, the electrode immersed in methanol/acetic acid solution to remove the template. The effect of $\mathrm{pH}$ and cross-linker/ monomer ratio was studied and was found that the $\mathrm{pH}$ range from 6 to 8.5 and cross linker/monomer molar ratio of 3 gave the best results, with shorter response times $(<4 \mathrm{~min})$, wider linear range 
$\left(3 \times 10^{-9}-7 \times 10^{-7} \mathrm{M}\right)$, and lower detection limit $\left(10^{-9} \mathrm{M}\right)$. From the influence of interfering substance, it can be seen that the concentration used in this study of interference was minuscule $\left(0.2 \times 10^{-7} \mathrm{M}\right)$ as a compared with heparin concentration $\left(0.2 \times 10^{-6} \mathrm{M}\right)$, so experiments with a higher level of interference will be needed to be sure that the sensor is not affected.

\subsubsection{Optical Sensors}

There are many applications that use the optical sensor. Table 2.2 represents the different techniques that were used in an optical sensor such as UV/Vis, fluorescence, chemiluminescence, surface Plasmon resonance, and Raman scattering [149].

Table 2.2. A summary of the technique was used by the optical sensors.

\begin{tabular}{|c|c|c|c|c|c|}
\hline Sensor Technique & Recognition System & Form & Analytes & LoD & Ref \\
\hline \multirow{3}{*}{ UV-VIS } & MIP-coated TiO2 & thin film & Lindane & $3 \times 10^{-8} \mathrm{M}$ & [150] \\
\hline & $\begin{array}{c}\text { PoPD-AuNPs/TIO2 } \\
\text { NTs }\end{array}$ & thin film & Chlorpyrifos & $0.96 \times 10^{-9} \mathrm{M}$ & [151] \\
\hline & MIP-NP & nanoparticles & Carbaryl & $1.6 \times 10^{-10} \mathrm{M}$ & [152] \\
\hline \multirow{7}{*}{ Fluorescence } & MIP-QDs & sol-gel particles & cytochrome & $4.1 \times 10^{-7} \mathrm{M}$ & [153] \\
\hline & MIP-QDs & nanoparticles & $\alpha$-fetoprotein & $7 \times 10^{-7} \mathrm{M}$ & [154] \\
\hline & CDs@MIP & sol-gel & sterigmatocystin & $5.9 \times 10^{-8} \mathrm{M}$ & [155] \\
\hline & $\begin{array}{l}\text { MIP-coated Mn- } \\
\text { doped ZnS QDs }\end{array}$ & sol-gel & nicosulfuron & $1.1 \times 10^{-9} \mathrm{M}$ & [156] \\
\hline & $\begin{array}{l}\text { MIP-modified fiber } \\
\text { optic }\end{array}$ & $\begin{array}{l}\text { microvolume } \\
\text { sensor }\end{array}$ & bisphenol & $7.45 \times 10^{-12} \mathrm{M}$ & [157] \\
\hline & $\begin{array}{l}\text { GQDs-embedded } \\
\text { SMIP }\end{array}$ & sol-gel & Metronidazole & $15 \times 10^{-7} \mathrm{M}$ & [158] \\
\hline & mMIP@CD/QD & sol-gel & $\begin{array}{l}\text { Diniconazole } \\
\text { (DNZ) }\end{array}$ & $2 \times 10^{-8} \mathrm{M}$ & [159] \\
\hline Chemiluminescence & MIP-DDV & $\begin{array}{l}\text { paper-based } \\
\text { chip }\end{array}$ & dichlorvos & $2.7 \times 10^{-8} \mathrm{M}$ & [160] \\
\hline
\end{tabular}




\begin{tabular}{|c|c|c|c|c|c|}
\hline & DA-Si-MG-MIP & thin film & dopamine & $1 \times 10^{-8} \mathrm{M}$ & {$[161]$} \\
\hline & $\begin{array}{l}\text { P-MIP-MMP MIP- } \\
\text { grafted }\end{array}$ & Paper disks & pesticide & $10^{-11} \mathrm{M}$ & [162] \\
\hline & CdTe QDs-MIP & Micro-plate & deltamethrin & $3.56 \times 10^{-11} \mathrm{M}$ & {$[163]$} \\
\hline \multirow{6}{*}{$\begin{array}{l}\text { Surface } \\
\text { resonance }\end{array}$} & BFA-MIP L- & Nanofilm & L- BFA & $2 \times 10^{-6} \mathrm{M}$ & {$[164]$} \\
\hline & MIP-POF & Thin film & furfural (2-FAL) & $1.04 \times 10^{-7} \mathrm{M}$ & {$[165]$} \\
\hline & MIP-POF & Thin film & L-nicotine & $10^{-8} \mathrm{M}$ & {$[166]$} \\
\hline & POF-MIP & Thin film & furfural(2-FAL) & $9.4 \times 10^{-6} \mathrm{M}$ & {$[167]$} \\
\hline & $\begin{array}{l}\text { MIP-AA polyaniline } \\
\text { film }\end{array}$ & Thin film & Ascorbic Acid & $1.28 \times 10^{-10} \mathrm{M}$ & {$[168]$} \\
\hline & $\mathrm{Ag} / \mathrm{MIP}$ & Thin film & profenofos & $6.7 \times 10^{-15} \mathrm{M}$ & {$[169]$} \\
\hline \multirow{10}{*}{ Raman scattering } & Nicotine-MIP & $\begin{array}{c}\text { Microsphere } \\
\text { particles }\end{array}$ & nicotine & $3 \times 10^{-4} \mathrm{M}$ & {$[170]$} \\
\hline & MIP-PFPA & nanoparticles & propranolol & $7.7 \times 10^{-4} \mathrm{M}$ & {$[171]$} \\
\hline & MIP-SERS & fine particles & melamine & $1.2 \times 10^{-5} \mathrm{M}$ & {$[172]$} \\
\hline & MMIPs & nanoparticles & rhodamine & $2.1 \times 10^{-6} \mathrm{M}$ & {$[173]$} \\
\hline & MMIPs & nanoparticles & phenylalanine & $2.4 \times 10^{-6} \mathrm{M}$ & {$[174]$} \\
\hline & Ag@MIP & core-shell & $\mathrm{RhB}$ & $10^{-10} \mathrm{M}$ & {$[175]$} \\
\hline & Ag@MIP & $\begin{array}{c}\text { Hybrid } \\
\text { nanoparticles }\end{array}$ & $\mathrm{RhB}$ & $10^{-12} \mathrm{M}$ & {$[176]$} \\
\hline & Ag@MI & $\begin{array}{c}\text { core-shell } \\
\text { nanoparticles }\end{array}$ & R6G & $10^{-14} \mathrm{M}$ & {$[177]$} \\
\hline & $\mathrm{Ag} @ \mathrm{MI}$ & core-shell & $\begin{array}{c}4- \\
\text { mercaptobenzoic }\end{array}$ & $10^{-15} \mathrm{M}$ & {$[178]$} \\
\hline & $\begin{array}{c}\text { MIP-glycoprotein- } \\
\text { AgNP }\end{array}$ & $\begin{array}{c}\text { macroporous } \\
\text { monolithic } \\
\text { structure }\end{array}$ & Glycoproteins & $13.8 \times 10^{-6} \mathrm{M}$ & {$[179]$} \\
\hline
\end{tabular}

\subsubsection{Environmental applications}

During their study, Casis, and et al. [180] fabricated a nonporous material based on MIP to detect the progesterone in water. The characterization of the film was studied. To determine the efficiency and specificity of the MIP, swelling ratio was examined which is 
considered a mentioned measurement on the cross-linking. FTIR and HPLC were used to assess the ability of the MIPs to capture the target molecule. The maximum imprinting capacity was found equal to 3.15 at a ratio of 1:0.1 molar between monomer/cross linker. However, interferences like organic matter and total suspended solids were not investigated.

Foguel and et al. [181] studied the detection of a textile dye, basic red 9 (BR9). Using an optical sensor resulting from a MIP-based evanescent wave fiber. The method used was cheap and robust. The MIP was synthesized by bulk polymerization, where 2acrylamido-2-methyl-1-propanesulfonic acid (AMPSA) was used as a monomer and EGDMA as a crosslinker. Light absorption by the MIP indicated more dye was bound to the MIP. Six types of dye were used to test the behavior of equilibrium binding at different $\mathrm{pH}$ levels of $2,5,7$, and 10 . The optimum time was $40 \mathrm{~min}$ with a detection limit equal to $2 \times 10^{-6} \mathrm{M}$. The BR9- MIP showed a more specific binding for BR9 than NIP. Nonetheless, the sensor would needs to be tested in real industrial textile effluents to prove their efficiency.

Moreover, Xue and co-workers [182] used another optical sensor technique to detect the bisphenol A in surface water and sport drink. Surface-enhanced Raman scattering (SERS) was used based on the surface-imprinted core-shell Au nanoparticles. The core-shell of MIP on the Au nanoparticles surface provided specific binding sites for the template, which causes the enhancing of SERS signals. The prepared MIP-AuNPs were added to the mixture solution of water and DMSO with heated for $5 \mathrm{hr}$ in $170{ }^{\circ} \mathrm{C}$ to eliminate the template. The signals of SERS were logged by a concentrated diode laser at $785 \mathrm{~nm}$ on the nanoparticles MIPs for the 30s per spectrum. The concentration of bisphenol 
A was between $2.2 \times 10^{-6}-9.98 \times 10^{-5} \mathrm{M}$ with a detection limit of $5.26 \times 10^{-7} \mathrm{M}$. The time to equilibrium was $10 \mathrm{~min}$. Although approach supplied efficient and fast method to detect the bisphenol A in water, SERS is a laboratory instrument, and it cannot be adapted for field use.

In another approach, MIP fluorescence sensor was prepared for determination of bisphenol A (BPA) in river water samples [183]. For this sensor, the carbon dots (CDs) was made in one step reaction by autoclaved $\mathrm{N}$-( $\beta$-aminoethyl $)-\gamma-$ aminopropyldimethoxysilane (AEAPMS) at $240 \mathrm{c}^{\circ}$ for $2 \mathrm{hr}$. BPA was dissolved in anhydrous ethanol and APTES as a monomer, TEOS as a cross linker, ammonia solution, and ultrapure water were added. The solution was left at room temperature for $24 \mathrm{hr}$ in the dark. Then, the MIP - coated CDs was collected by centrifugation and washed with ethanol to remove the reagent. Consequently, the template was removed by ethanol/water solution $(\mathrm{v} / \mathrm{v}=1: 1)$. The fluorescence emission decreased as the concentration of BPA increased in the MIP@CDs. The detection limit of BPA for MIP@CDs was $0.3 \times 10^{-7} \mathrm{M}$, the linearity response was $10^{-7}-4.2 \times 10^{-6} \mathrm{M}$ and the response time below $1 \mathrm{~min}$. The sensor showed excellent reproducibility with stability of 1 month at room temperature. Also, the sensor selectivity was tested with virous interference substances such as phenol, o-nitrophenol (oNP) and paranitrophenol (4-NP) and showed a good selectivity.

A chitosan/graphene oxide - magnetite - molecularly imprinted polymer (Cs/GMMIP) was combined with CdTe quantum dots@luminol nanoparticles to detect chrysoidine, an industrial dye. Chemiluminescence was utilized in this method. The CdTe QDs@luminol was applied to amplify the signal of chemiluminescence, and the Cs/GM were used to increase the adsorption on the surface of MIP because of the amino and 
hydroxyl group. The binding affinity experiments to the Cs/GM-MIP showed that the adsorption increased with increased chrysoidine concentration. The CdTe QDs@luminolCs/GM-MIP-CL sensor appeared sensitive for the detection of the target in real samples. The absorption capacity was $8.9 \mathrm{~mol} / \mathrm{g}$ with a linear range of $10^{-7}-10^{-5} \mathrm{M}$ and a detection limit of $32 \times 10^{-9} \mathrm{M}$ [184]. However, the authors identify possible interferences with coexisting substances.

\subsubsection{Biomedical applications}

Ciprofloxacin, a fluoroquinolone antibiotic, has been selectively detected using a Magnetically Imprinted Surface Enhanced Raman Scattering (MI-SERS) optisensor [185]. In this approach, three techniques were used in combination: magnetic separation, molecularly imprinted polymer, and the surface enhanced Raman scattering. The results of these techniques were a novel method with a high extraction level and extremely sensitive detection. The MIP was applied as a coating to cover the magnetic particles. The nanoparticles were used firstly in solutions with different concentrations of ciprofloxacin, and an outer magnet was used to separate them. The detection limit for this method was $10^{-9} \mathrm{M}$. In addition, a magnetic chip was applied to the fetal bovine serum, and the detection limit was $10^{-7} \mathrm{M}$.

In another work, a MIP film was deposited on a gold film for detection of histamine by surface plasmon resonance [186]. The MIP film was prepared by dissolving of MAA and EGDM in DMSO with the target molecule histamine and then the polymerization solution was degassed under ultrasound. Next, the thin film gold chip was coated by the spin-coating. Finally, the template was removed after polymerization by using a 
methanol/acetic acid solution. The optisensor showed high selectivity, sensitivity, and reproducibility toward histamine as a compared with the NIP material. The measured resonant angle shift increased with the increase of histamine concentration in the range of 25-1000 $\mu \mathrm{g} / \mathrm{L}$, and the detection limit for the sensor was $25 \mu \mathrm{g} / \mathrm{L}$.

\subsubsection{Acoustic sensors}

This type of sensors uses piezoelectric materials which convert the mechanical signal into an electrical one [187].The piezoelectric materials can be natural like quartz or manufactural like lithium tantalite (LiTaO3). Acoustic wave sensor can be divided into three types according to the guiding mechanism of acoustic wave [188]. First, the bulk acoustic wave (BAW) which the wave transfers randomly through the volume of the material. Many applications were done in this field [189]-[198] used the quartz crystal microbalance $(\mathrm{QCM})$ to detect the difference templates in different applications ( Environmental and Biomedical) which used the same principle of the ones used BAW. Second, surface acoustic wave (SAW) [199]-[202] in which the wave transfers on the surface of materials. Third, acoustic plate wave which transfers by reflecting from the surfaces. The last two waves can be work together as a surface generated acoustic wave (SGAW). There are different applications for SGAW with MIP like sher horizontal-surface acoustic wave (SH-SAW) [203], love wave [204]. The measurement of the sensor relied on the calculated the frequency shift for each concentration:

$\Delta f=f i-f_{0}$

Where, $f_{\mathrm{o}}$ is steady resonant frequency, and $f i$ frequency of the sensor. 


\subsubsection{Environmental applications}

Liang and co-workers [189] developed an easily portable acoustic sensor to detect the herbicide 2-4 dichlorophenoxyacetic acid $(2,4-\mathrm{D})$ in water. At the start, the particle of the MIP was prepared and mixed with PVC powder in tetrahydrofuran. After that, the solution was dropped onto the center of Ag electrode with rotation to spread on the surface. The electrode was left at room temperature which made the tetrahydrofuran evaporate, and the MIP particles were immobilized on the BAW. At the same time, NIP-BAW was prepared as a reference electrode by the same procedure without the template $2,4-\mathrm{D}$. The optimum $\mathrm{pH}$ for the sensor was found equal to $(\mathrm{pH}=7)$ because the difference between MIP sensor and reference sensor (NIP) was very high at this $\mathrm{pH}$. The effect of the coating was also studied, and they found that the significant amount of MIP in coating or high coating rate would affect on the binding capacity. The optimal amount of MIP to PVC in the coating was found equal to 3.5. Moreover, 4 structural analogs to the 2,4-D were studied to investigate the selectivity of the sensor. The sensor showed very high selectivity, and linearity in the range $6.0 \times 10^{-7}-1.0 \times 10^{-4} \mathrm{M}$. The limit of detection was $2.0 \times 10^{-7} \mathrm{M}$. The instruments used for the MIP-BAW sensor are straightforward and cheap as a compared with HPLC, GC. However, the authors did not mention about the stability and reproducibility of the sensor.

Tancharoen and co-workers [193] devised a successful sensor to detect the explosives 2,4,6-trinitrotoluene (TNT) and 2,4-dinitrotoluene (DNT). The sensor was prepared by using different monomers (AAM, MAA, and MAAM); EGDMA was used as cross-linker and AIBN as an initiator. The same monomer was used for both TNT and DNT because they have similar chemical structure except the TNT has one more nitro group 
than DNT. The sensitivity of the sensor was studied for the TNT-MIP in the ratio $(1: 6: 25$, TNT, AAM, and EGDMA). The experiments showed that the sensitivity changed according to the modification of TNT concentration.

A MIP film coated a quartz resonator sensor was utilized successfully for determination of trace microcystin-LR (MC-LR) in drinking water [194]. The polymerization solution was prepared by mixing of MC-LR, MAA, DMSO, EGDMA, and AIBN. After cleaning in a hot-air oven for $15 \mathrm{hr}$ at $60 \mathrm{c}$, the chip was immersed in the polymerizing solution to form a thin film of MIP. The MC-LR template was extracted by rinsing the film with an ethanol-acetic acid solution $(9: 1, \mathrm{~V} / \mathrm{V})$. Finally, the polymerized chip was washed with deionized water and dried under nitrogen. The sensor had a limit of detection (LOD) of $0.4 \times 10^{-7} \mathrm{M}$, and able to reproduce results with a difference in signal less than $2.58 \%$. However, the authors did not clarify the effect of $\mathrm{pH}$ on the sensor, nor interferences like organic matter and total suspended solid to resemble other water samples.

\subsubsection{Biomedical applications}

Peng and co-worker [191] successfully applied an acoustic sensor with a molecularly imprinted polymer to detect pyrimethamine in serum and urine samples. The MIPs were fabricated by mixing the pyrimethamine as a template with MAA as a monomer and EDMA as a crosslinker with the found of AIBN as an initiator in benzyl alcohol and acetonitrile. The mixture was transferred to a glass polymerization tube and the unwanted or excesses gasses removed by pumping of nitrogen gas. The tube was then sealed and polymerized in a water bath at $60{ }^{\circ} \mathrm{C}$ for $24 \mathrm{hr}$. The obtained polymer was crushed and sieved to get MIP particles with a diameter of $1 \mu \mathrm{m}$. After that, $20 \mathrm{mg}$ MIP particles were 
suspended in $20 \mathrm{ml}$ tetrahydrofuran containing $10 \mathrm{mg}$ of PVC with stirring. The mixture was spread on the Ag electrode to obtain the sensor. The sensor showed very high selectivity and good sensitivity. The optimal $\mathrm{pH}$ was found to be 8.5 . The team was successful to devise a sensor required simpler and cheaper instrument as a compared with sensor used HPLC, GS, or UV methods.

Heparin-induced thrombocytopenia (HIT) has been measured at a surface imprinted polymer (MIP) on quartz crystal microbalance with dissipation (QCM-D) [195]. This approach was considered the first step to prepare the platelet imprinting. The MIP film was made following multi-steps procedure. First, the polymer solution was prepared by mixing AIBN, 1-Vinyl-2-pyrrolidone (VP), divinyl benzene (DVB), acetone, N-methyl pyrrolidone, and di- methylformamid (DMF) in reaction vials. Next, the prepared solution was spin coated on the front QCM electrode, and the coated electrode was polymerized by using UV light to form a semi-solid gel film. After that, the template (platelet suspension) was spin coated with the film. Moreover, the coated electrode was completed by polymerizing again under UV light. Finally, the template was detached from the electrode by deionized water at $40{ }^{\circ} \mathrm{C}$. The results showed the dissipation shift was higher in plateletrich plasma (PRP) by 55\%. Also, the dissipation shift of HIT positive is more important than the negative controls with healthy donor sera and heparin by 4-5 times.

\subsection{Conclusions and Future work}

Molecularly Imprinted polymers have significantly diversified during the last decades both in term of materials used and applications. This technique was applied in different fields. Sensors are one of the most critical applications which were described in this review. Different type of polymerization was briefly described. Also, the various types 
of physical forms were reported. Three types of sensors were described with their applications in environmental and biomedical. The electrochemical sensors are widely used in both applications, however, the main problem of these sensors that it recognized by low selectivity and sensitivity sometimes.

The acoustic sensors were identified as straightforward and cheap, but the selectivity and reproducibility were not mentioned well in the articles. The third type of sensor was the optical sensor which can detected with a different kind of instruments. However, almost all of the instrument used in reports are complicated, and training is needed. A sensor that requires a simple straightforward instrument is still needed to overcome the problem of expensive and complicated tools used. Also, rugged and/or supported materials are test, which can be used in the field. Additional desirable characteristics are reusability to lower the cost of sensor use, and low detection limits. For biomedical application, fast response is highly desirable, to allow for fast diagnosis and better outcomes in treatments. 


\section{Chapter Three}

\section{Photonic Molecular Imprinted Polymer Film for the Detection of \\ Testosterone in Aqueous Samples}

\subsection{Introduction}

Testosterone is the primary androgen hormone which has a significant effect on human health [205]. The average level of testosterone in a healthy adult male is (300-1000) $\mathrm{ng} / \mathrm{dl}$ [206]. It has been used illegally by athletics, as results of anabolic effect which is related to muscle mass and strength growth [207]. The low concentration of testosterone makes it challenging to find a suitable, efficient, and economical method of detection and quantification. Nowadays, many analytical techniques are used to monitor testosterone in urine and blood samples such as GC-MS [208], [209], Isotope-Dilution Gas Chromatography-Mass Spectrometry ID/GC-MS [210], LC-MS-MS [211]. These methods are complicated, expensive, time consuming, and training required [212], which leads to the need for a simple, rapid, and low-cost method to detect the testosterone in aqueous samples.

Molecularly imprinted polymers (MIPs) constitute a feasible alternative. Molecular Imprinted (MI) is a technology that allows the fabrication of a polymer material with the ability to selectively adsorb the target molecules as a result of the highly specific sites formed during fabrication [208]. The key steps to the process of molecularly imprinted polymers include: integration of the target into the monomer solution, polymerization to carefully design the spatial distribution of template molecules inside the polymeric matrix, template removal to provide cavities have the same morphology of the target, and rebinding 
the target in a mechanism similar to a "key-lock" principle of enzymes [113]. Synthetic MIPs have shown advantages over their biological counterparts including improved stability, simple preparation, excellent ability to capture small molecules, reusability and longer shelf-lives [213].

several methods were used to analyze the testosterone using MIPs, mostly as an adsorption or concentrating media in combination an analytical instrument for quantification, including microring resonator [205], HPLC-MS-MS [214], HPLC-SPE [215], SPME- GC-MS [216], LC-MS-MS [217]. However, these methods are still complicated, expensive and time consuming. An optical sensor based MIPs is a promising method, since it does not need a second analytical step. The methodology relies on the adsorption of target molecule, i.e. testosterone to an optical active thin film and the change the light reflectance properties that is produced after adsorption to detect the binding of the target [218].

In this work, a photonic sensor based on a combination of colloidal crystal from silica nanoparticles, molecularly imprinted polymers, and inverse opal technique was fabricated. First, a thin MIP porous film was obtained with a morphology corresponding to the inverse of a silica particles colloidal crystal. Secondly, an optical sensor (supported MIP film) was developed and tested. This optical sensor had the ability to swell or shrink in aqueous solution upon molecular recognition or environmental conditions leading to a change in the optical properties. Unsupported MIP characterization and validation experiments were conducted aided by HPLC determination of testosterone in the test samples, prior to the use of the supported MIP film to detect the template by reflectance. The technique was 
used for the first time to create a testosterone optical sensor, with detection and quantification capabilities in aqueous samples.

\subsection{Materials and Methods}

Tetraethoxysilane (TEOS), ammonia solution (25\% in water), ethanol (99.5\%, 200 proof), acrylic acid (AA) (99\%,) ethylene glycol dimethacrylate (EGDMA) (98\%), 2,2'azobisisobutyronitrile (AIBN) (98\%), testosterone (99\%), bisphenol A (99\%), flutamide (100\%) and 17 beta-estradiol (98\%) were supplied by Sigma-Aldrich, St. Louis, MO, USA. Hydrofluoric acid (HF) (49\%), acetic acid (96\%), acetonitrile and phosphate buffer solution were purchased from Fisher, Fair Lawn, NJ, USA (https://www.fishersci.com). Ultra-pure water $\left(18.2 \mathrm{M} \Omega^{*} \mathrm{~cm}\right.$ at $\left.25^{\circ} \mathrm{C}\right)$ was obtained from a Thermo Scientific ${ }^{\mathrm{TM}}$ Barnstead $^{\mathrm{TM}} \mathrm{E}-$ Pure $^{\mathrm{TM}}$ Ultrapure Water Purification System (Waltham, MA, USA). All chemicals were purchased as reagent grade and used without further purification.

The methodology is schematically shown in Figure 3.1. A colloidal crystal, fabricated by vertical self-assembly and secured between two slides, was infiltrated by the polymerization solution and polymerized under UV-light. Immersion in HF resulted in detachment of the glass and removal of the silica particles. When two glass slides were used, unsupported films were obtained, while PMMA-supported MIPs were fabricated when the second slide was plastic. The unsupported material was used for the characterization and binding experiments, when the concentration of testosterone in solution was measured by HPLC; the photonic properties were investigated in the PMMA supported films. 


\subsubsection{Synthesis of porous MIPs}

Silica particles were synthesized by a modified Stöber method [84]. Briefly, ethanol $(200 \mathrm{~mL})$ was mixed in a flask and stirred with ammonia $(22 \mathrm{~mL})$ at $300 \mathrm{rpm}$ for approximately $10 \mathrm{~min}$. Then, TEOS $(14 \mathrm{ml})$ was added rapidly and the mixture allowed to react for 12 hours. Particles obtained were centrifuged and re-dispersed in ethanol once and in distilled water twice to remove residual reactants. For the fabrication of colloidal crystals, a clean microscope glass slide (Fisher Scientific, Pittsburgh, PA, USA) with dimensions $1 \times 13 \times 76 \mathrm{~mm}$ was placed vertically in a vial containing a suspension of silica particles (volume fraction $0.1 \%$ ) in ethanol; after the volatilization of ethanol, colloidal crystals were self-assembled on both sides of the glass slides.

The MIP films were fabricated using a non-covalent self-assembly approach, by filling the void volume in the colloidal crystal with a polymerization solution. The solution was prepared by dissolving $5 \mathrm{mg}$ of testosterone in $2 \mathrm{ml}$ of acetonitrile; The functional monomer AA or MAA $(400 \mu 1)$ was added and the liquid stirred in the dark for 3 hours. Then, 500 $\mu 1$ of crosslinker EGDMA and $9 \mathrm{ml}$ of AIBN as initiator, were added. A second clean glass slide was placed on top on the one containing the colloidal crystal and the two slides were firmly held together forming a "sandwich". One end of the sandwich structure was put in contact with the polymerization solution and the liquid allowed to rise by capillary forces until it completely filled the void volume within the colloidal crystal. The polymerization was completed under UV light $(\lambda=365 \mathrm{~nm}$, Cole Parmer lamp, I $=2$ $\mathrm{mWcm}^{-2}$ ) for $8 \mathrm{~h}$ at room temperature. Non-imprinted polymers (NIPs) were prepared as a control following the same procedure as MIPs with the exception of testosterone addition. 


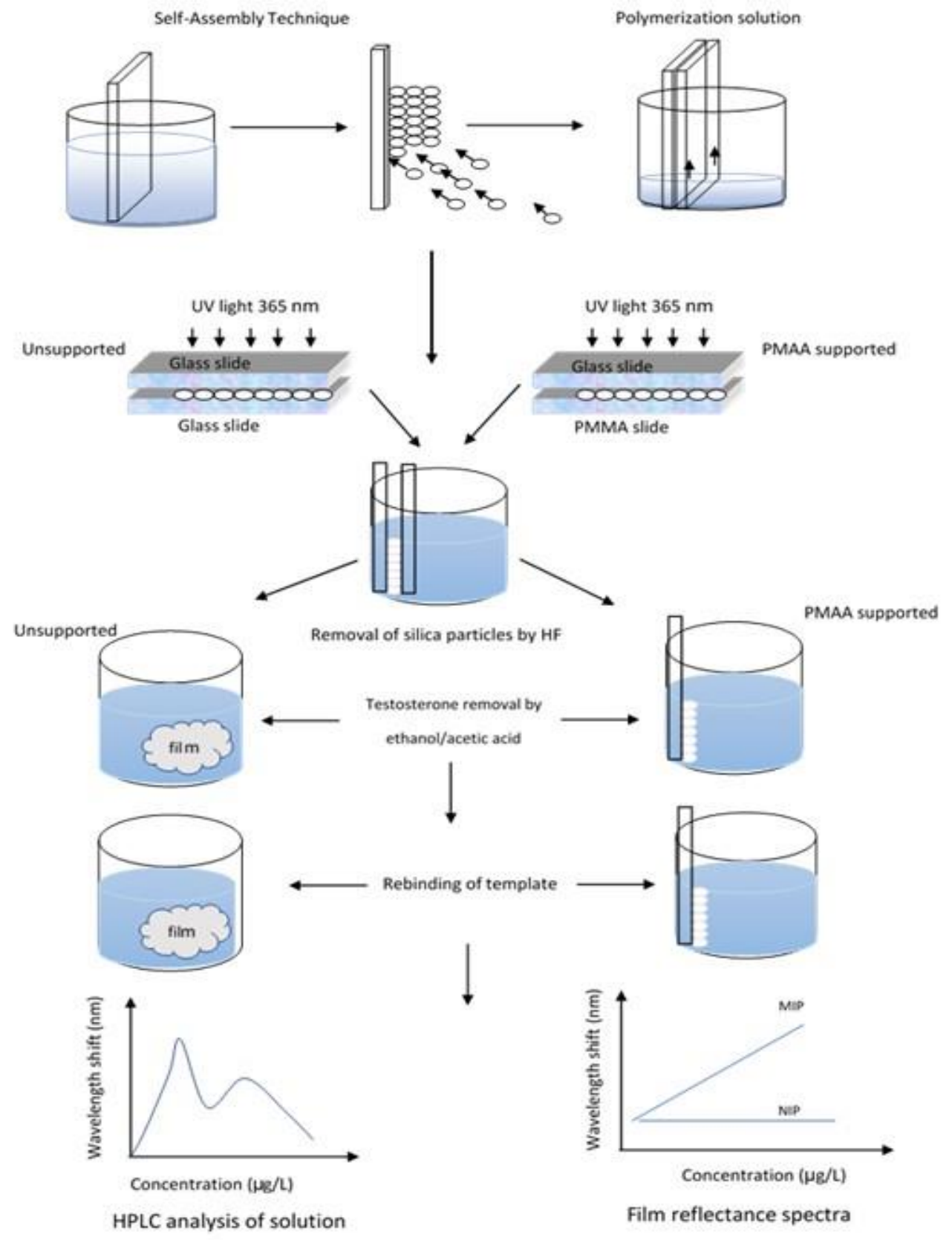

Figure 3.1. Schematic representation of the fabrication process and research design.

The silica particles, now entrapped within the polymer, were etched away by immersing the system in 5\% HF for 12 hours, followed by exhaustive rinsing with DI water. This step also produced the detachment of the film from the glass slides. Finally, the resulting films were washed with 1:1 (v:v) acetic acid: ethanol solutions for 30 minutes to 
remove the testosterone. The washing procedure was repeated 6 times, after which the films were subjected to a final rinse with ethanol to remove any remaining acid. Supported thin films were fabricated following a similar methodology as described above for the selfstanding polymers, but in this approach, poly (methyl methacrylate) (PMMA) slides were used as the second slide to make the "sandwich" structure; additionally, the elution of the testosterone was conducted using 1:9 (v:v) acetic acid: ethanol, changing the solvent every $30 \mathrm{~min}$ for $5 \mathrm{~h}$, since the solution used for the unsupported MIPs damaged the PMMA slides. The inverse opal film was firmly attached to the surface of PMMA support slides.

\subsubsection{Characterization}

The size and morphology of silica particles and the porous films were investigated by electron microscopy using a FEI Quanta 600 FEG Environmental Scanning Electron Microscopy (ESEM). The specimens were mounted on stubs using carbon tape and covered with a thin conductive layer of gold (1.5 - 3.0 nm) using a Emitech K575x sputter coater. ImageJ software (National Institutes of Health, NIH) was used to analyze the SEM images. In the determination of particle size, at least 300 particles were measured from 3 different images. The size of silica particles was confirmed by Dynamic Light Scattering (DLS) on a Zetasizer Nano ZS instrument (Malvern Instruments).

Equilibrium swelling experiments were conducted on NIPs, synthesized from AA and MAA, in phosphate buffer at two pHs (6 and 8), at $25^{\circ} \mathrm{C}$. The polymers were swollen in solutions for 72 hours at room temperature to reach equilibrium, and the degree of swelling was determined gravimetrically. The $\%$ swelling ratio $(\% S R)$ was calculated from the following expression: 


$$
\% S R=(m s-m d) / m d
$$

where $m_{s}$ is the mass of the swollen film at equilibrium and $m_{d}$ the mass of the lyophilized films.

Fourier Transformed Infrared Spectroscopy (FTIR) spectra were collected in a Cary 660 spectrometer (Agilent Technologies, Santa Clara, CA, USA) between the wavelengths of $4000-400 \mathrm{~cm}^{-1}$.

The recognition binding experiments were conducted by weighting $5 \mathrm{mg}$ of lyophilized MIP or NIP film in testosterone solutions of variable concentration, from 0.1 ppm to $20 \mathrm{ppm}$, for $24 \mathrm{~h}$ when equilibrium was assumed. The residual concentration of the solution was determined by HPLC, and the recognition capacity was calculated from equation 2:

$$
R C=(C i-C e) V t / m
$$

where $\mathrm{Ci}, \mathrm{Ce}$ are the initial and the final concentrations of testosterone (in $\mathrm{mg} \mathrm{mL}^{-1}$ ), respectively; $V t$ is the volume of solution (in $\mathrm{mL}$ ), and $m$ is the mass of the film (in $\mathrm{g}$ ). The imprinting polymer efficiency (IE) was calculated as:

$$
I E=\frac{R C_{M I P}}{R C_{N I P}}
$$

where $R C_{M I P}$ and $R C_{N I P}$ are the recognition capacities of MIP and NIP, respectively.

The kinetics of testosterone binding was investigated for unsupported MIPs in 5ppm testosterone solutions. During incubation, samples were taking periodically and residual testosterone concentration measured by HPLC. Additionally, the kinetics of the 
optical response (shift of the Bragg's diffraction peak) of supported films in a $1 \mathrm{ppm}$ testosterone solution and was assessed by collecting reflectance spectra for the film periodically during incubation.

The desorption of testosterone after incubation was analyzed using unsupported films. Once equilibrium had been reached (i.e., 24 hours incubating time), the films were incubated in pure water for another $24 \mathrm{hrs}$ and then the liquid analyzed for testosterone. The experiment was conducted with initial testosterone concentrations between $0.1 \mathrm{ppm}$ and 1 ppm.

\subsubsection{Analytical Method}

The testosterone solutions were analyzed by High-Performance Liquid Chromatography (HPLC) in a Shimadzu LC-2010A HT. A Kinetex® C18 stainless steel column with $2.6 \mu \mathrm{m}$ particles size, $100 \mathrm{~mm}$ length $\mathrm{x} 4.6 \mathrm{~mm}$ internal diameter was used. The mobile phase consisted of $0.1 \%$ phosphoric acid in water (A) and $100 \%$ acetonitrile (B). The gradient conditions were $0-0.5 \mathrm{~min}, 2 \% \mathrm{~B} ; 0.5-7 \mathrm{~min}, 2-80 \%$ (linear gradient) $\mathrm{B}$; 7.0-9.0 min, 80-98\% (linear gradient) B; 9.0-10.0 min(linear gradient) , 2\% B; 10.0-15.0 $\min , 2 \% \mathrm{~B}$ with a flow rate of $0.5 \mathrm{ml} / \mathrm{min}$. The system was washed first with $50 \%$ acetonitrile and $50 \%$ of $1 \%$ phosphoric acid, and the column was washed with $2 \%$ acetonitrile and $98 \%$ of $1 \%$ phosphoric acid solution. The injection volume was $20 \mu 1$, detection was performed by UV-vis adsorption at a wavelength of $265 \mathrm{~nm}$; the technique has a detection limit of $10 \mathrm{ppb}$ 


\subsubsection{Reflectance analysis}

Testosterone adsorption into the binding sites resulted in a change in Bragg diffraction of the polymer due to swelling or shrinking of the hydrogel film and refractive index changes, and therefore a clear optical signal can be detected. UV-vis reflectance spectra of the films were recorded and their respective $\lambda_{\max }$ was correlated to solution concentration. The diffraction peak $\lambda_{\max }$ for the porous hydrogel is given by the Bragg equation:

$$
\lambda_{\max }=1.633\left(\frac{d}{m}\right)\left(\frac{D}{D \mathrm{o}}\right)\left(\mathrm{n}_{\mathrm{a}}{ }^{2}-\sin \theta^{2}\right)^{0.5}
$$

where $d$ is the sphere diameter of the colloidal silica particle, $m$ is the order of Bragg diffraction, $\left(D / D_{0}\right)$ is the degree of swelling of the gel $\left(D\right.$ and $D_{0}$ denote the diameters of the gel in the equilibrium state at a certain condition and in the reference state, respectively), $n_{a}$ is the average refractive index of the porous gel at a particular condition, and $\theta$ is the angle of incidence.

The reflectance of the films was measured over a wavelength range of 200-800 nm, using a double-beam UV-visible spectrophotometer (Cary 60, Varian) with a Harrick Scientific's Specular Reflection Accessory (ERA-30G) for measurement of reflectance at a fixed angle of 30 degrees.

\subsection{Results}

\subsubsection{Characterization of silica particles and films}

The monodispersed silica particles were prepared with careful control of the reaction conditions in order to achieve the desired monodisperse size distribution. Figure 3.2a, b shows representative SEM images of the top view and cross section of the obtained 
colloidal crystals. Size measurements from the SEM images (top views only) showed a narrow size distribution of the particles, with an average radius of $330 \mathrm{~nm}$ and standard deviation of $8 \mathrm{~nm}$. Only particles with a standard deviation below $5 \%$ were used in the deposition experiments.

The average particle size was confirmed by DLS measurements, that gave an average hydrodynamic diameter of $375 \pm 7 \mathrm{~nm}$, and a polydispersivity index of 0.012 . The two measurements are in reasonably good agreement considering experimental error and the fact that DLS commonly overestimate size due to the effect of the solvent in the hydrodynamic diameter.

Colloidal crystals were prepared by vertical self-assembly from $0.1 \%$ volume fraction suspensions; the particle concentration was chosen to achieve a desired film thickness. Jiang et al. [219] established that the relationship between initial volume fraction of the nanoparticle suspension and the thickness of the self-assembled crystal can be described by Equation 5:

$$
K=\frac{\beta L \emptyset}{0.605 d(1-\varnothing)}
$$

Where $\mathrm{K}$ is the number of layers, $\mathrm{L}$ is the meniscus height, $\beta$ is the ratio between the velocity of a particle in solution and the fluid velocity and is taken to be $1, \mathrm{~d}$ is the particles diameter, and $\emptyset$ is the particle volume fraction in solution. The calculated the number of layers according to equation 5, with $\mathrm{L}=3500 \mu \mathrm{m}, \emptyset=0.001$ and $\mathrm{d}=330 \mathrm{~nm}$, was 17, while the number of layers observed from SEM images was about 13-15 layers. 
A thicker film will offer a larger total surface area, and therefore, more binding sites, but diffusion into the lower cavities will slow down equilibration of the film, and consequently, the response. Furthermore, as colloidal crystal grew thicker, they became unstable and partially detached from the slides, leading to faulty films. Thus, the thinner film that produces a measurable response within the range of values of interest would represent the optimal condition. The average number of silica particle layers observed in the colloidal crystals and the measurements of the silica particle diameters were used to estimate the thickness of the MIPs at approximately $5 \mu$.
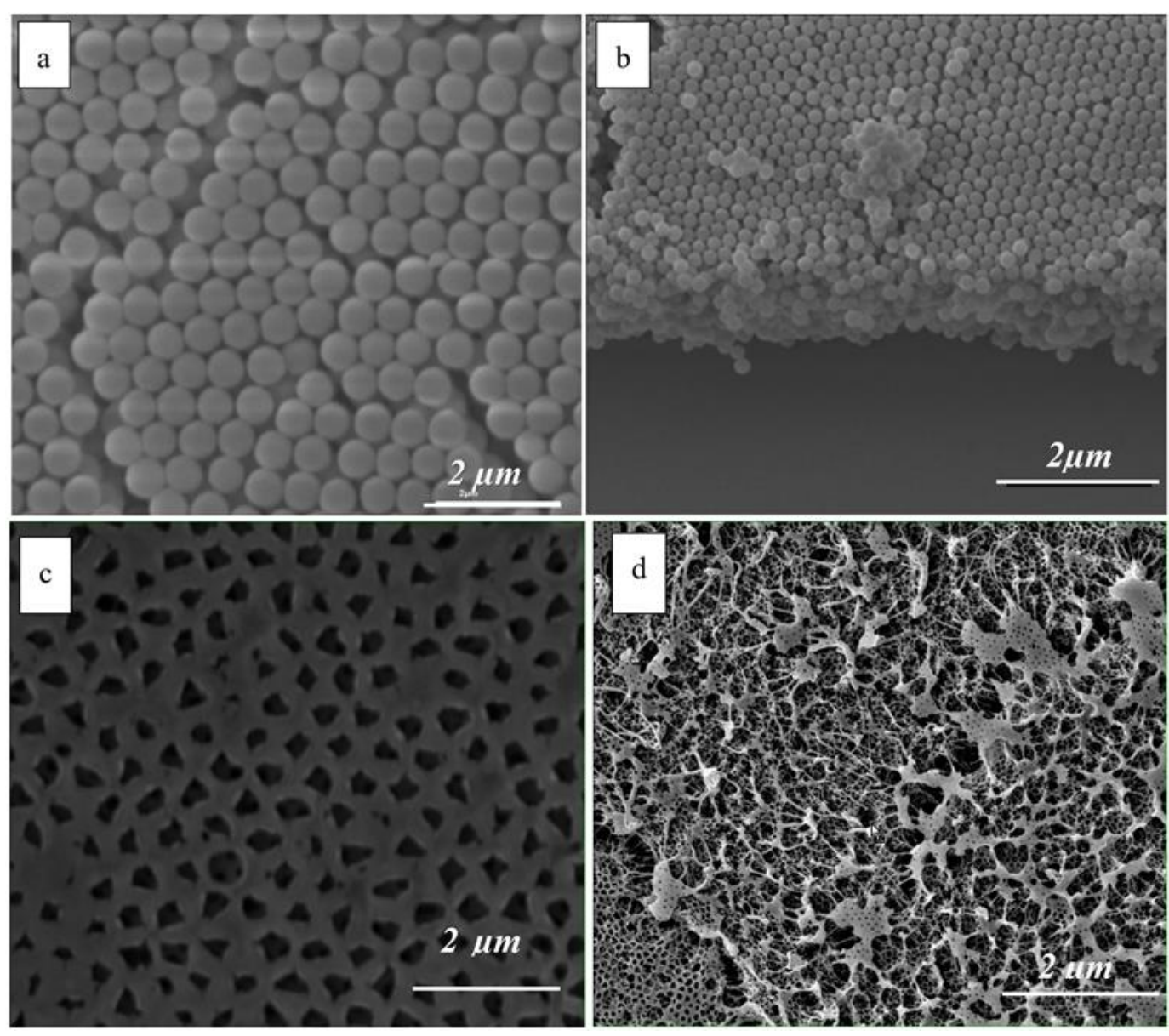

Figure 3.2 SEM images of: (a) colloidal crystal top layer; (b) colloidal crystal showing cross section; c) porous structure (top view) of the MIP s film; (d) internal morphology of the MIP film. 
Figure 3.2c shows the surface of the photonic thin films after the particle removal and Figure 3.2d is representative of the internal pore structure.

Two functional monomers commonly used in MIP fabrication, AA and MAA, were tested to investigate the effect of their chemistry on MIP characteristics. The water contact angle for the polymers was $35 \pm 2^{\circ}$ and $59 \pm 4^{\circ}$ for AA and MAA films, respectively. MAA is less hydrophilic due to the methyl moiety than AA, but exhibits better mechanical properties, generally resulting in stronger films, more resistant during handling. A volume of $400 \mu \mathrm{l}$ of each monomer was added to the polymerization mixture $(0.006 \mathrm{mM}$ of $\mathrm{AA}$, $0.005 \mathrm{mM}$ of MAA). The films were prepared with identical amounts of template, cross linker, and initiator.

The materials were characterized with respect to their ability to adsorb water and swell. Higher water affinity is expected to lead to faster response, as transport of the target is facilitated while a higher degree of swelling translates into a larger variation in the photonic crystal structure which in turn is associated with a more significant optical response.

AA films presented high swelling ratios both at acidic and basic $\mathrm{pH}$ (Figure 3.3). SR value were lower at $\mathrm{pH} 6$ than at $\mathrm{pH} 8$, most noticeable for MAA. Both polymers are expected to be present in their ionic form, with pKa of 4.26 and 4.65 for AA and MAA, respectively [220], [221]. However, at the higher $\mathrm{pH}$, the carboxyl group is deprotonated to a higher extend, leading to electrostatic repulsion that causes the polymer to swell more.

The contribution of non-polar groups (methyl in MAA) to the overall hydrophobicity of the polymer was outweighed at higher $\mathrm{pH}$ by the charge developed as a 
result of ample deprotonation of the carboxylic groups. The higher SR observed for AA are consistent with the more hydrophilic character of the polymer.

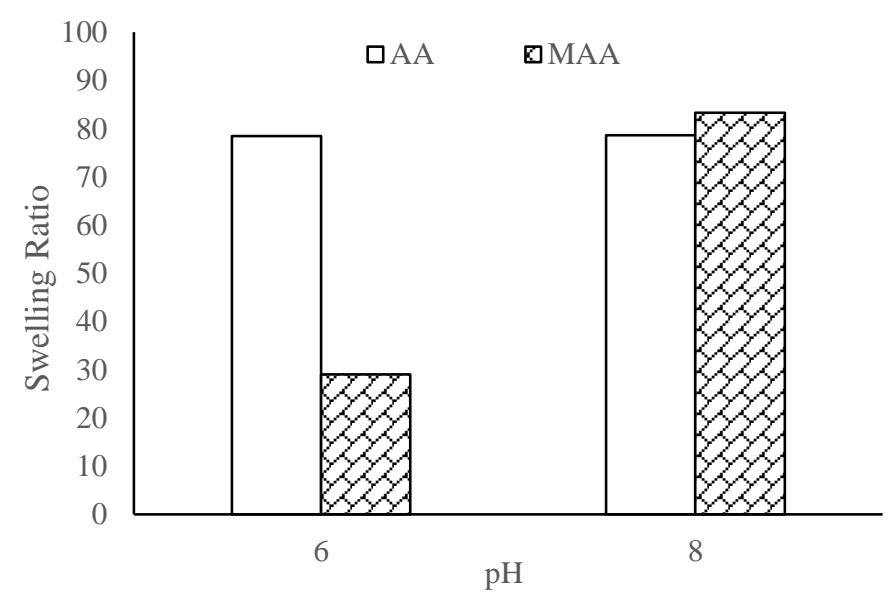

Figure 3.3. Effect of $\mathrm{pH}$ on Swelling Ratio for polyacrylic acid films (clear bars) and polymet hacrylic acid films (shaded bars).

The chemical functional groups and their interactions with the testosterone molecule after polymerization were investigated by FTIR. The films before and after testosterone removal and the non- imprinted films (NIPs) i.e. same material and morphology but no target molecule added, showed a similar IR spectrum, but with differences in intensity of the bands (Figure 3.4). MIPs after removal of testosterone had higher intensity of bands compared to those before target removal and NIPs. All the imprinted cavities in the MIP before template removal were occupied by testosterone molecules which lowered the absorbance of functional groups involved in the non-covalent binding; when testosterone was washed from the polymer, the groups were free to vibrate and therefore, showing higher adsorption intensity [222]. The spectrum contains various bands, the peak at around 2400 corresponds to the $\mathrm{OH}$ group of the carboxylic acid, which is very clearly observed in the MIP samples after 
target removal. The disappearance of this group in MIP + testosterone samples as well as the NIPs indicates the formation of the interaction between the carboxylic group of the polymer and testosterone during binding. In addition, the peak at $1610 \mathrm{~cm}^{-1}$ may be attributed to the carbonyl group $(\mathrm{C}=\mathrm{O})$ of testosterone, as it was not present in the washed MIPs and NIPs spectra [223].

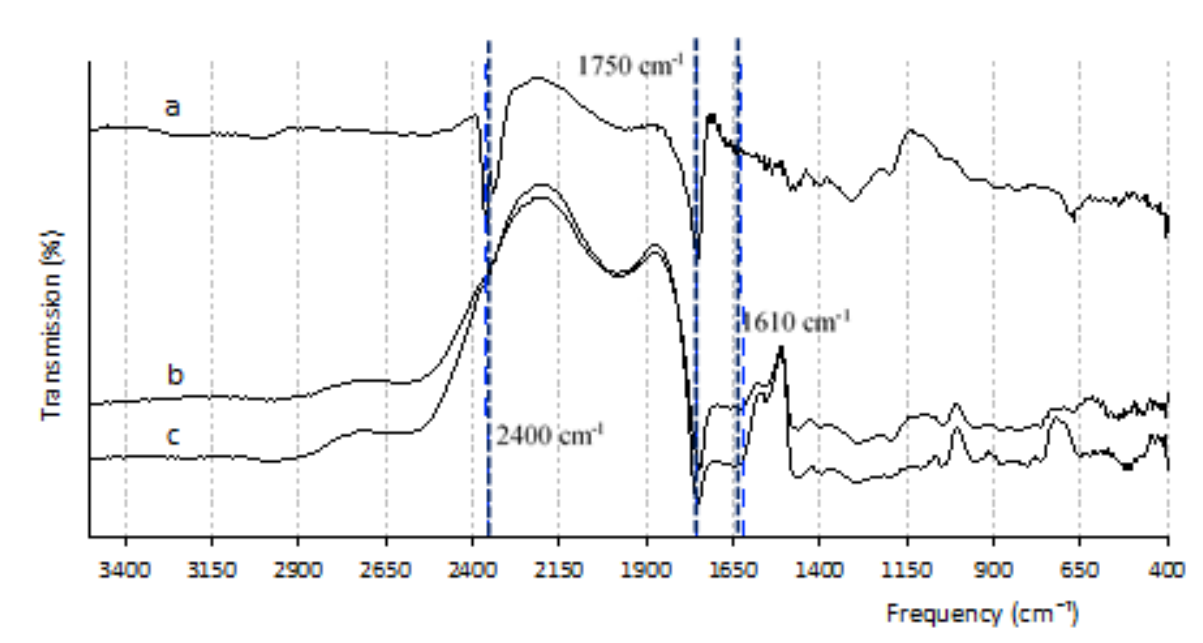

Figure 3.4. FTIR spectra of the films at different synthesis stages: a) MIP after testosterone removal, b) NIP, c) MIP before testosterone removal.

$\mathrm{RC}$ and IE were calculated from the equilibrium incubation experiments and summarized in Table 3-1. The AA films presented consistently higher RC and IE than MAA films, and therefore selected as the material to be used in subsequent experiments.

AA MIPs were further tested in an extended concentration range (Figure 3.5). The MIPs exhibited higher affinity for the template than the NIPs at all testosterone concentration tested. The RCs increased linearly with concentration, both at a higher, between $1 \mathrm{ppm}$ to $20 \mathrm{ppm}$, and lower range, $0.1 \mathrm{ppm}$ to $1 \mathrm{ppm}$. 
Testosterone adsorption on the NIP films was lower than for MIPs in all cases, but significant relative to the amount of MIP rebinding for incubations at $1 \mathrm{ppm}$ and below, resulting in poor IF values. The IF reached 10 for the higher concentration of $20 \mathrm{ppm}$, and declined to 1.6 at the lowest concentration tested, $0.1 \mathrm{ppm}$. An average value for IF of AAMIPs was calculated to be 3.16 .

Table 3.1. Recognition Capacity and Imprinting Efficiency of AA and MAA MIPs.

\begin{tabular}{|c|c|c|c|}
\hline \multirow{3}{*}{ MAA } & $\begin{array}{c}\text { Concentration } \\
(\mathrm{ppm})\end{array}$ & $\begin{array}{c}\mathrm{RC} \\
(\mathrm{mg} / \mathrm{g})\end{array}$ & $\mathrm{IF}$ \\
\cline { 2 - 4 } & 1 & 0.21 & 1.23 \\
\cline { 2 - 4 } & 5 & 0.51 & 1.1 \\
\hline \multirow{3}{*}{ AA } & 10 & 0.9 & 1.5 \\
\cline { 2 - 4 } & 1 & 0.22 & 1.71 \\
\cline { 2 - 4 } & 5 & 0.98 & 2.13 \\
\cline { 2 - 4 } & 10 & 2 & 2.77 \\
\hline
\end{tabular}

${ }^{1}$ average value of at least double samples.
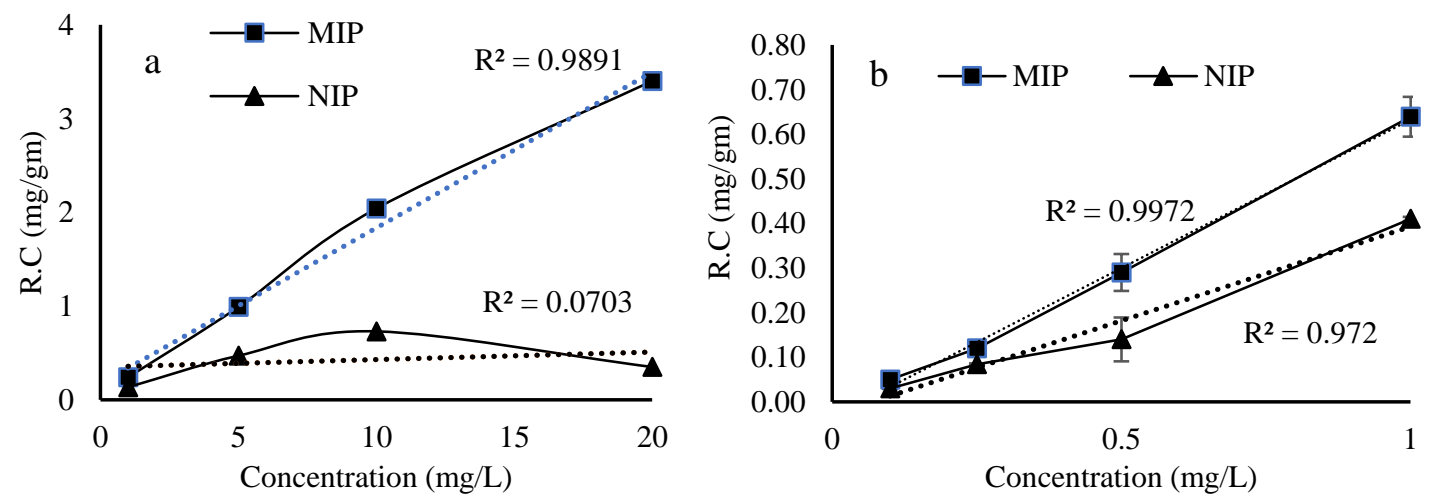

Figure 3.5. Recognition Capacity for AA-MIP and AA-NIP at higher (a) and lower (b) range concentrations.

The kinetics of the testosterone attachment were investigated in order to determine the minimum time required for the MIP to reach equilibrium with the incubation solution. Negligible concentration changes were determined after 60 minutes of incubation for MIPs 
(Figure 3.6a). The stability of the bound testosterone was tested by desorption experiments, where the previously equilibrated films were exposed to pure water. The results showed that the attachment was stable, and minimal transfer of testosterone to the liquid phase was observed (Figure 3.6b). The results showed that the attachment was stable, and minimal transfer of testosterone to the liquid phase was observed (Figure 3.6b). The lowest amount of testosterone detected after incubation in pure water was $0.083 \mathrm{ppm}$ which may represent nonspecific adsorbed material as attachment between the target molecule and the MIP in nonspecific adsorption is generally weaker than rebinding to the imprinted sites. The concentration of testosterone in the final incubation solutions were fairly insensitive to concentration in the initial solution and quickly reached a plateau at $0.1 \mathrm{ppm}$, which follows a similar behavior as adsorption on NIPs, and reinforces the assumption of being nonspecifically adsorbed molecules. The films were left to dry at room temperature before the second incubation, but a contribution by liquid trapped inside the porous structure cannot be completely ruled out.
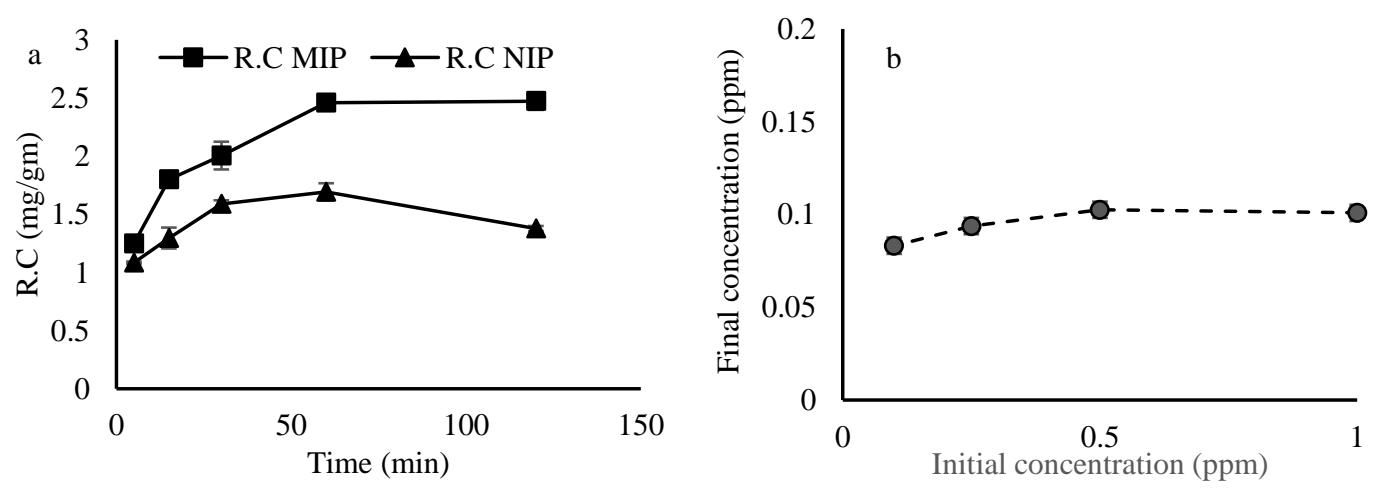

Figure 3.6. Kinetics of rebinding to unsupported MIP and NIP films, (a) initial testosterone concentration 5 ppm; (b) desorption of testosterone from MIP films initially incubated at variable concentrations, $0.1 \mathrm{ppm}$ to $1 \mathrm{ppm}$ testosterone 


\subsubsection{Reflectance Analysis}

A simple and inexpensive method for detection of the testosterone stems from the photonic properties of the MIP [224]-[228]. The periodicity of the porous film resulted in interference in reflection of the incident light. For each film, the wavelength of reflection was correlated to the amount of rebinding of the target; with increasing concentration of testosterone in the incubation solution, the peak wavelength of the reflected light was further shifted to longer wavelengths. As the imprinted nanocavities became increasingly occupied by the target, the internal pore structure of the inverse opal was altered, producing a different optical response (Figure 3.7). A clear difference could be observed between the behavior of MIPs and NIPs. When MIPs were exposed to increasing concentration of T, the peak of the reflected light gradually shifted to longer wavelengths. However, there was almost no peak shifting for the NIP under the same conditions. We were able to detect low concentrations of testosterone by this technique, from $5 \mathrm{ppb}-100 \mathrm{ppb}$ (Figure 3.7), which is within the normal range of hormone level in adult males. The detection limit was determined to be $4.2 \mathrm{ppb}(\mathrm{S} / \mathrm{N}=3)$.

The kinetics experiment of the optical response due to the rebinding showed that the peak wavelength became constant for times longer than 30 minutes' incubation in the test solution (Figure 3.8). The stability of the material was evidenced by the reversibility of the wavelength shifts observed after six cycles of use and regeneration (Figure 3.9). The clean MIP was promptly recovered after incubating in a $1 \mathrm{ppm}$ solution by eluting the testosterone from MIP using ethanol/acetic acid in a ratio 9:1. The highly open the internal pore structure of the film facilitated the back diffusion of the testosterone molecules during 
washing, and a remarkable degree of regeneration of the initial conditions of the film was achieved [229].
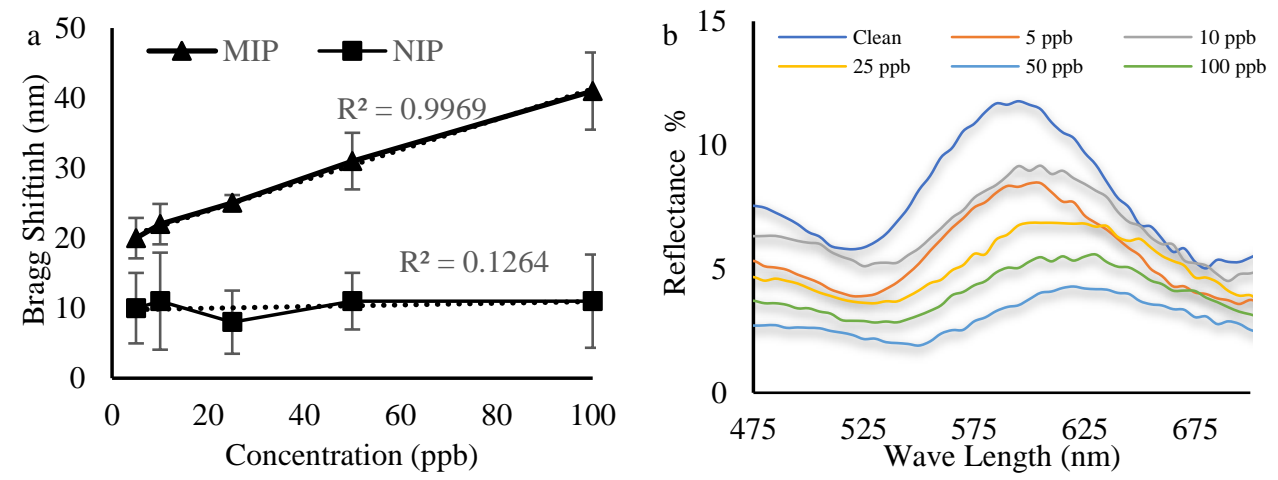

Figure 3.7 Optical properties supported MIPs: (a) diffraction peak shift for MIPs and NIPs after incubation at various concentration of testosterone; (b) reflectance spectra of supported MIPs after incubation at various concentration of testosterone.

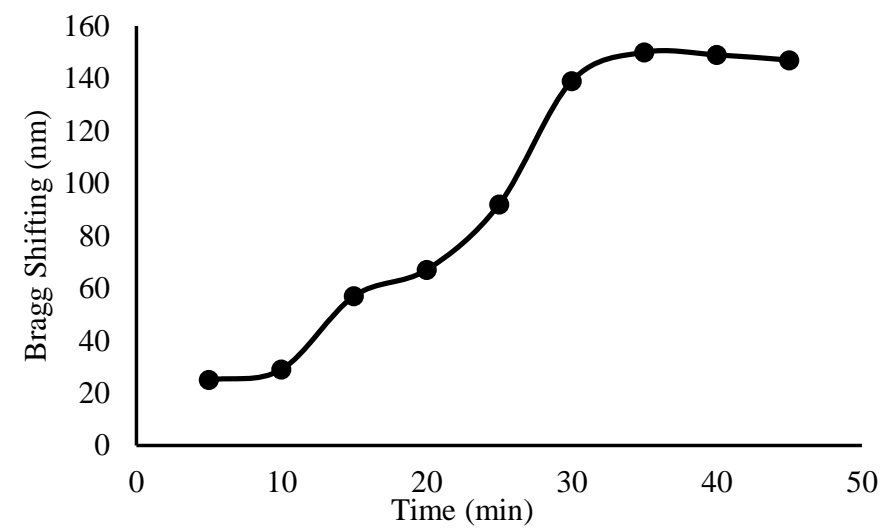

Figure 3.8. Kinetic response of the testosterone MIP

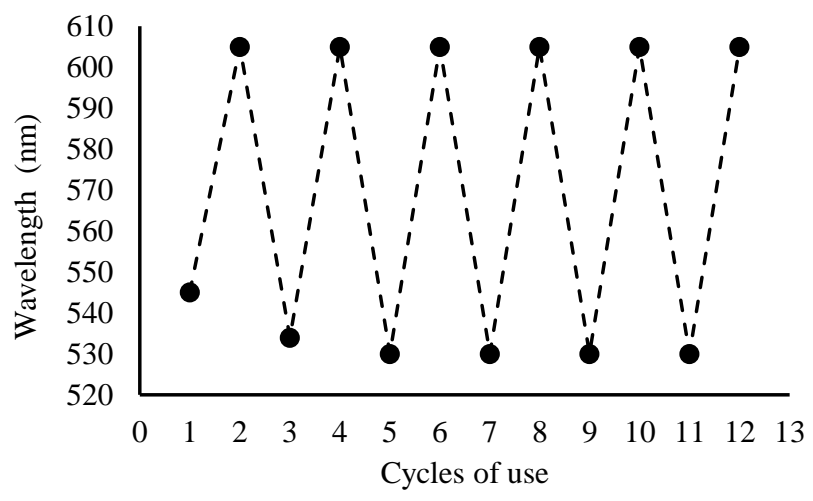


Figure 3.9 Recoverability of photonic sensor for 6 cycles of use and regeneration.

Chemicals other than testosterone can potentially bind into the imprinted cavities, resulting in errors due to interferences. To investigate the competitive recognition ability of the MIPs, the response to testosterone was compare to that of a group of selected compounds: estradiol, flutamide, and bisphenol A. Estradiol was selected due to its similar structure to testosterone, while the other two are known to be androgenic endocrine disrupting chemicals, i.e. they interfere with natural receptors of testosterone. The MIPs were tested with different concentrations between $5 \mathrm{ppb}$ and $100 \mathrm{ppb}$ of these chemicals. MIPs were dipped into each solution for 30 minutes, and spectra was recorded to determine the peak wavelength shift.

The MIP exhibited different recognition capacity for testosterone and other substances tested (Figure 3.10). Because of the structure similarities, estradiol induced a response from the MIPs close to that of testosterone. However, selectivity towards testosterone was significantly better when the other two compounds were tested.

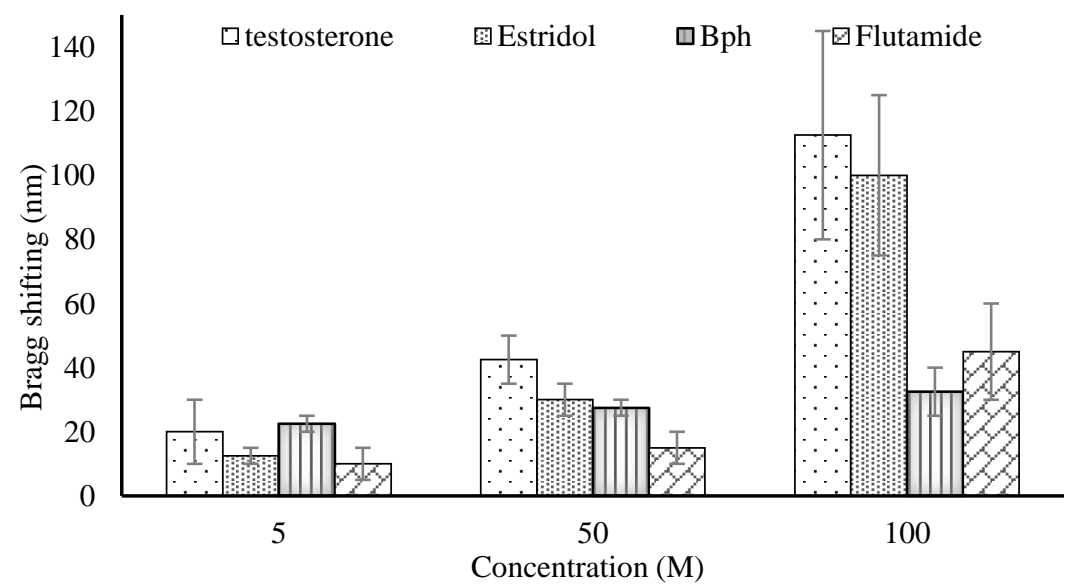

Figure 3.10. Optical response of the MIPs to testosterone and selected compounds. 


\subsection{Conclusion}

Inverse opal imprinted polymers were fabricated from monodisperse silica particle crystals. The thin films were supported on PMMA slides for easy handling as the MIPs can be used to determine concentration of testosterone, the target molecule, in unknown liquid samples. Although polyMAA results in stronger films, polyAA films proved to be better for the intended application, evidenced by the significantly higher imprinting efficiency, their hydrophilicity and superior water adsorption characteristics. Mechanical strength was not critical as the films are supported in the external material.

The recognition capacity varied linearly with concentration in a wide range of testosterone levels, although using different linear calibration curves based on range is recommended. Some degree of non-specific adsorption was observed for NIPs, in all cases in a much lower amount than in MIPs. Testosterone adsorption reached equilibrium after 60 minutes of incubation with tests solutions. However, the optical response of the film presented improved characteristics in relation with its function as testosterone sensor. The diffraction peak wavelength shifted when the MIPs were incubated in testosterone solutions; the shift was measurable after exposures to concentrations down to $5 \mathrm{ppb}$, reaching the reference levels of testosterone in blood for adult males. The NIPs showed no significant wavelength shift, which implied that the methodology is unaffected by nonspecific adsorption. Moreover, the peak wavelength becomes stable after 30 minutes' incubation time, an important improvement over the previous determination. Other hormones with similar chemical structure have the ability to interfere in the accurate determination of testosterone with the fabricated MIPs, although the effect can be minimized if the context of the sample is known, i.e. expected estradiol vs. testosterone 
concentration. Our test experiments represented a worst case scenario, when the response of the interference is compared at the same concentration and in the absence of testosterone. For samples with unknown reference values of hormone concentration, a combination of MIPs targeting each one of the analogs (estradiol, estrogen, testosterone) may provide additional information and help differentiate target from interfering compounds. 


\section{Chapter 4}

\section{A novel Sensor to Detect of Testosterone(T) in Human Blood Based on Molecularly Imprinted Hydrogel}

\subsection{Introduction}

Testosterone $(\mathrm{T})$ is a natural steroidal androgen responsible for anabolic, androgenic, and virilizing effects on the human body, as well as the development of secondary sexual characteristics. T blood tests are needed for males that suffer from low T levels because of illness or aging. Additionally, T plays a key role in the muscle mass increase and the gain of muscle strength and therefore has been used by the athletes to enhance their performance and increase strength despite being prohibited by Word Antidoping Agency [223].

A number of studies have been published on methods to detect $\mathrm{T}$ in human samples such as plasma [230], serum [210], [231], saliva[232], urine [233], hair[234], [235] and nails[236]. Clinically, immunoassays are the most common method for routine analysis. However, this technique is a laboratory-based, time-consuming method that lacks sufficient sensitivity and specificity, in particular for the lowest concentrations of $\mathrm{T}$ that occur in children and women [211]. Chromatographic techniques, such as HPLC[237], GC-MS [238], and LC-MS-MS[239] become the gold standard analytical method because of high specificity and accuracy. However, they are time-consuming, expensive, and complicated [240]. Due to the high cost of the gold standard method, patients often go untested or not tested as often as they need to be for optimal clinical care. 
Many studies have attempted to overcome the aforementioned challenges by using biosensor methods to detect $\mathrm{T}$. Biosensors could offer a method for rapid and low-cost measurements. To date, various types of biosensors, such as surface plasmon resonance, electrical, interferometric, and photonic crystal-based sensors, have been utilized as diagnostic devices [207], [215], [229], [241]-[243]. A photonic sensor is a tool that depends on the optical signal to detect the contaminants. Photonic crystal sensors have several advantages with respect to the other biosensors, including inexpensive fabrication and short detection time. There is currently no point of care (POC) medical devices for the assessment of $\mathrm{T}$ levels in blood in the market and a thorough search of the scientific literature resulted in no previous reports of the photonic molecularly imprinted polymer (MIP) sensor for the detection of $\mathrm{T}$ in blood.

In this work, a molecularly imprinted polymer technique was used to prepare a photonic sensor to detect the level of $\mathrm{T}$ in human blood in a fast and accurate manner. A novel sensor based on molecularly imprinting technology was prepared. After the success of the sensor to detect the testosterone in water, the sensor was tested with horse blood to investigate the sensor response to the different concentrations of T. Finally, the sensor was tested with human blood. The goal of this work is to develop a tool that can provide the results of testosterone fast, easily and accurately. Our sensor will permit the patients and the physicians to use the sensor at home or the clinic when they need to detect the level of $\mathrm{T}$.

\subsection{Materials and methods}

\subsubsection{Materials}


Chemicals were obtained from Sigma-Aldrich (St. Louis, MO) and used without further purification; ethanol (200 proof, ACS reagent, 99.5\%), acrylic acid (AA) (99\%), ethylene glycol dimethacrylate (EGDMA) (98\%), 2,2'-azobisisobutyronitrile (AIBN) (98\%), testosterone (T1500) (98\%), Hydrofluoric acid (HF) (49\%), dihydro T (DHT or 5aDHT) (97\%), hydrocortisone (Co) (98\%), dehydroepiandrosterone (DHEA) (98\%), dehydroepiandrosterone sulfate (DHEAS) (98\%), estradiol (E2) (98\%), and androstenedione (ANDROS) (98\%).

Commercial silica particles were purchased from (Pinfire - Gems \& Colloids, German) with a diameter of $300 \mathrm{~nm}$. Glass microscope slides with dimensions $1 \times 13 \times 76$ $\mathrm{mm}$ were obtained from Fisher Scientific (Pittsburgh, PA, USA). Polymethylmethacrylate (PMMA) slides were purchased from ePlastic (San Diego, CA, USA) and cut into the same dimension as the glass slides. Human blood samples from an unidentified donor, and horse blood samples from Hemo State Laboratory, CA, USA were used. Ultra-pure water (18.2 $\mathrm{M} \Omega \cdot \mathrm{cm}$ at $25{ }^{\circ} \mathrm{C}$ ) was used in all experiments and obtained from a Thermo Scientific ${ }^{\mathrm{TM}}$ Barnstead $^{\mathrm{TM}}$ E-Pure ${ }^{\mathrm{TM}}$ Ultrapure Water Purification System (Waltham, MA, USA).

\subsubsection{Fabrication of porous MIP film}

\subsubsection{Preparation of colloidal crystal slides}

Colloidal crystals made of silica particles were obtained by self-assembly techniques [240]. A $0.1 \%$ volume fraction suspension of silica particles was prepared, stirred overnight, and then sonicated for two hours. The colloidal crystals were obtained by placing $25 \mathrm{ml}$ of silica particle suspension in a beaker. Glass slides were carefully cleaned with ethanol and immersed vertically in the silica particle suspension inside a furnace at 50 
$\mathrm{C}^{\circ}$ for $24 \mathrm{hrs}$. As the solvent evaporated, particles self-assembled forming colloidal crystals on both sides of the glass slides.

\subsubsection{Synthesis of Molecularly Imprinted Photonic Hydrogel (MIPH)}

The imprinted polymer was obtained in the void spaces inside the colloidal crystal to produce a sensor with pre-specified morphology. The pre-polymerization mixture consisted of $\mathrm{T}$ as a template, AA as a monomer, EGDMA as a crosslinker, and AIBN as initiator. A molar ratio of template/ monomer/ crosslink (1/32/8) was used. Initially, 57.6 $\mathrm{mg}$ of $\mathrm{T}$ was dissolved in $1 \mathrm{~mL}$ of ethanol and a volume of $439 \mu \mathrm{l}$ of AA was added. The mixture was stored in the dark at $4^{\circ} \mathrm{C}$ for 3 hours to allow for the complexation of the monomer and the target molecule. After that, $302 \mu \mathrm{L}$ of EGDMA and 13mg of AIBN were added to the precursor solution and then sonicated for 5 min. PMMA slides were first cleaned with a laboratory grade anionic detergent solution at $70{ }^{\circ} \mathrm{C}$ and then with ethanol before using. Afterward, a glass slide containing colloidal crystals on both sides was placed between 2 PMMA slides to form a sandwich-like arrangement, holding the three slides together with a clip. One edge of the three slide arrangement was put in contact with the pre-polymerization solution. As a result, the solution infiltrated the interstitial space of the silica colloidal crystals under a capillary force. The slides were held together for 10 mins to completely infiltrate the crystal and then the solution was polymerized under UV light at $\lambda=365 \mathrm{~nm}$. In order to prevent the temperature of the samples to increase during polymerization, an ice bath or gel back was placed beneath the slides, with aluminum foil separating the two. The samples felt cold to the hand throughout the polymerization process, thus no thermal damage to the hydrogel was expected. 
After polymerization, the three slide assembly was placed in a freezer at $-18{ }^{\circ} \mathrm{C}$ for 40 minutes, which allowed to separate the glass slides. Then, the PMMA slides containing the hydrogel films were immersed in 5\% hydrofluoric acid for $48 \mathrm{hrs}$ to remove the silica particles. Finally, the slides were rinsed by 1:9 (v:v) acetic acid/ethanol for 5 cycles of 30 min each with gentle stirring to elute the $\mathrm{T}$ from the polymer.

\subsubsection{Material characterization}

The size of the silica particles and the morphology of the colloidal crystals were studied by using FEI Quanta 600 FEG Environmental Scanning Electron Microscopy (ESEM). The samples were mounted on a metal stub using carbon tape and coated with 1.5-3 nm of Gold by an Emitech K575x sputter coater (Quorum Technologies Ltd., Ashford, Kent, UK). Esprit software 2.1 (Bruker, USA) was used to confirm the size of silica particles. The software can be used to detect, measure and analyze any form of feature. Top views of the colloidal crystals were analyzed using the software to calculate the size distribution of the particles. Several SEM images with at least 300 silica particles in each were investigated by the software. The pore morphology of the fabricated films was also characterized by SEM.

\subsubsection{Sensor optical response}

An optical method was used to measure the capture of T by the MIP films. The reflectance spectrum of MIPs was recorded using a double beam UV-VIS spectrophotometer (Cary 60, Varian, Palo Alto, CA, USA) with a Harrick Scientific's Specular Reflection Accessory (ERA-30G) at a fixed angle of $30^{\circ}$. 
The kinetics of the sensor response was investigated with $5 \mathrm{ppb}$ and $10 \mathrm{ppb} \mathrm{T}$ solutions. Experiments were conducted in triplicates, at least three reading in each sensor at each separate concentration of $\mathrm{T}$ were tested for a different period and the diffraction spectra were collected and analyzed.

A calibration curve relating the optical response of the MIPs and the concentrations of $\mathrm{T}(1,5$, and $10 \mathrm{ppb})$ was obtained. The same sensor was inserted in different concentrations, starting from the lowest and moving up to the highest level; Bragg diffraction spectra was recorded at each condition.

\subsubsection{Analysis of interferences by structurally similar hormones}

The selectivity of the MIP binding sites is given by the original templating with the target molecule, testosterone. Therefore, those sites will exhibit a steric preference for $\mathrm{T}$, but also will result in favorable chemical interactions between polymer matrix and rebound molecule, stemming from the original orientation of the $\mathrm{T}$ molecule when complexed with the functional monomer. Six hormones that have a chemical structure similar to T, (DHT, DHEA, Co, DHEAS, $E_{2}$, and ANDROS), were chosen to study the response of the sensors to the interferences. Their chemical structures are shown in Figure 4.1.

In these tests, three different concentration levels of the potential interfering hormones, $0.04 \mathrm{ppb}, 5 \mathrm{ppb}$, and $30 \mathrm{ppb}$, were used with a background $\mathrm{T}$ concentration of $5 \mathrm{ppb}$ and $150 \mathrm{mM} \mathrm{NaCl}$ to mimic physiological level of ionic strength. To obtain a $\mathrm{T}$ standard curve response of the sensor, MIPs were incubated in $0.04 \mathrm{ppb}, 5 \mathrm{ppb}$ and $30 \mathrm{ppb}$ $\mathrm{T}$ solutions with a background ionic strength of $150 \mathrm{mM} \mathrm{NaCl}$. Then, the same sensors were 
incubated in the test solutions containing $\mathrm{T}$ and the interference to study the effect of each hormone on the detection of $\mathrm{T}$.

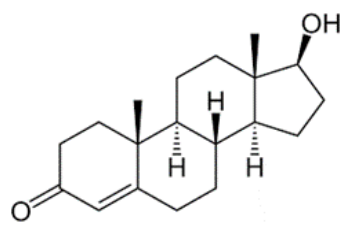

(a) $\mathrm{T}$

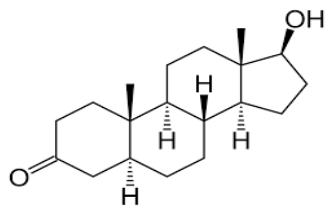

(b) DHT

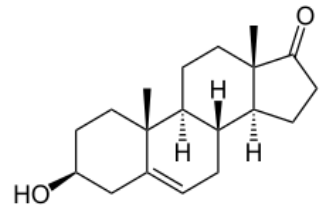

(c) DHEA

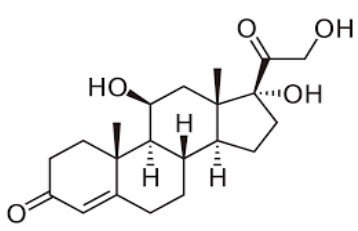

(d) $\mathrm{CO}$

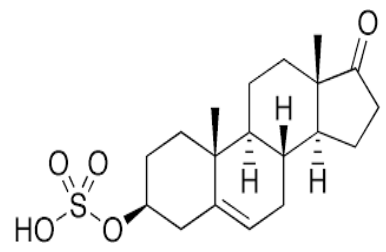

(e) DHEAS

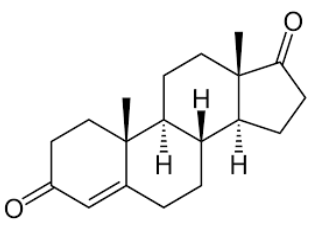

(f) $E_{2}$

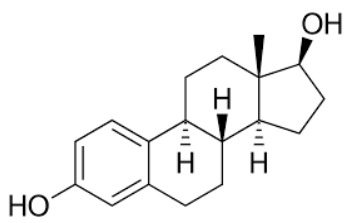

(g) ANDROS

Figure 4.1. Molecular structures of the T and interferences hormones; a) Testosterone b) dihydro testosterone

c) dehydroepiandrosterone d) hydrocortisone e) dehydroepiandrosterone sulfate f) estradiol g) androstenedione

\subsubsection{Molecular Modeling of Interference}

Molecular modeling was applied for the calculation of molecular potential energies for all hormones considered in this study, in an effort to predict interferences and identify chemical functionalities or structures that could affect sensor selectivity. Calculations were performed using Gaussian 09W software. The studied hormones were optimized applying the density functional theory (DFT) method with the hybrid Becke, three-parameter, LeeYang-Parr functional (B3LYP), and Pople's basis set 6-311G (2d,p)[244]-[246]. No symmetry constraints were imposed during the geometry optimization process. Vibrational frequencies were carried out to verify that geometries represented a minimum on the 
potential energy surface [245]. Solvent effects were included using SMD continuum solvation model[245]. The molecular electrostatic potential mapped onto the isodensity surfaces was visualized using GaussView 6 [247].

\subsubsection{Analysis of blood samples}

Blood samples (horse and human) were used to investigate the response of the sensors. Horse blood (HemoState Laboratory, CA, USA) spiked samples were prepared by adding different volumes of 2 and $20 \mathrm{ppm}$ of $\mathrm{T}$ solution. Volumes added were calculated to increase the concentration of $\mathrm{T}$ in the blood by 2, 22, 62 and $262 \mathrm{ppb}$.

Human blood was obtained from an anonymous donor. Human plasma samples were freshly used after extraction. To prepare the plasma samples, the human blood samples were centrifuged for $30 \mathrm{~min}$ at $2500 \mathrm{rpm}$. Aliquots of the samples were spiked with a 1,10 , and $30 \mathrm{ppb}$ solution of $\mathrm{T}$. A volume of $1.5 \mathrm{ml}$ of plasma was used for MIP incubation. After incubation, the sensor was rinsed with ultrapure water and the Bragg diffraction spectra was recorded.

The sensors were regenerated by rinsing with a solution of ethanol/ acetic acid with a ratio of 9/1 five times and incubated again to investigate their reusability. In order to validate the results of the samples, the samples were sent to the Mayo Clinic (M.C) laboratory to test the concentrations of testosterone. HPLC-MS/MS was used to investigate the testosterone concentration on the Mayo Clinic. Also, a deuterated stable isotope (d3testosterone) was used as an internal standard for quality control.

\subsection{Results and Discussion}




\subsubsection{Characterization of silica particles and MIP}

The SEM study of the silica particles showed that the average size was $300 \mathrm{~nm}$ and a standard deviation of the size distribution was $8 \mathrm{~nm}$ or $2.7 \%$ below the $5 \%$ cut off set to obtain a well-organized crystal.

Figure 4.2.a \& b shows a representative cross section and top view of the colloidal crystals as prepared. Figure 4.2.c depicts the morphology of a porous polymeric film fabricated using the colloidal crystal as template.
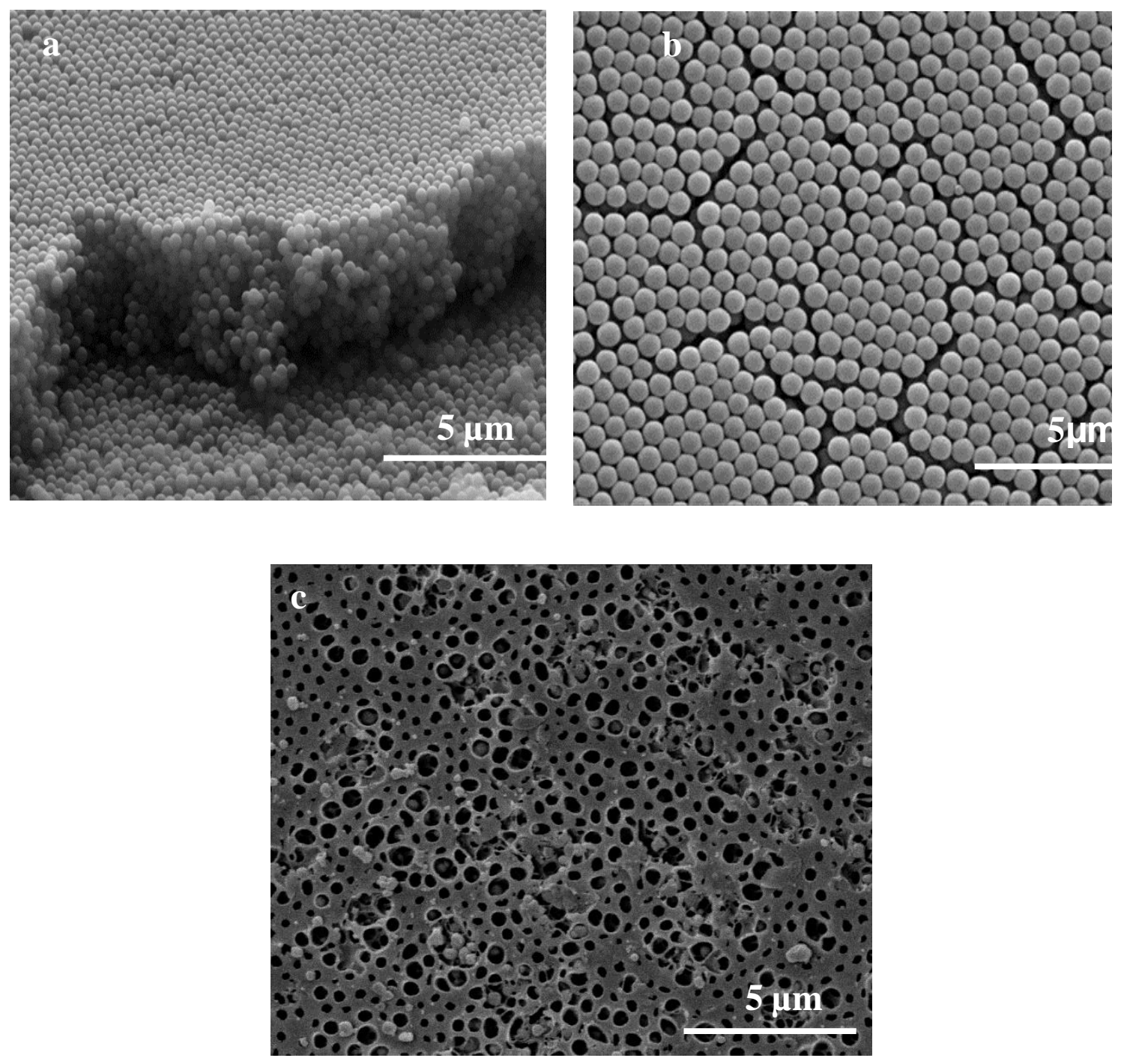
Figure 4.2. SEM image of (a) cross section of colloidal crystal, (b) Top view of silica particles colloidal crystal template (c) 2D structure of the MIP film.

\subsubsection{MIP response in water solutions}

When the hydrogel film is incubated in an aqueous solution, it will swell or shrink resulting in a wavelength shift of the peak of the reflectance spectra, that can be observed with a UV-Vis spectrophotometer. Moreover, if the target molecule is present in the solution, the peak shift will vary depending on its concentration. The rebinding of a T molecule into the cavities of the polymer causes a change in the diameter of the pore cavities of the hydrogel film, which leads to an increase in wavelength shift with increasing $\mathrm{T}$ concentrations. This produced clear, readable wavelength spectra of UV-Vis spectrophotometer according to the Bragg diffraction formula. The Bragg diffraction peak wavelength $\left(\lambda_{\max }\right)$ of the colloidal crystal was $538 \mathrm{~nm}$ (green), whereas, the peak wavelength for the MIP films before T removal shifted to $433 \mathrm{~nm}$, and furthermore to 380 $\mathrm{nm}$ (violet) after eluting the T. The colors can be observed in the digital images in Figure

\section{3.}
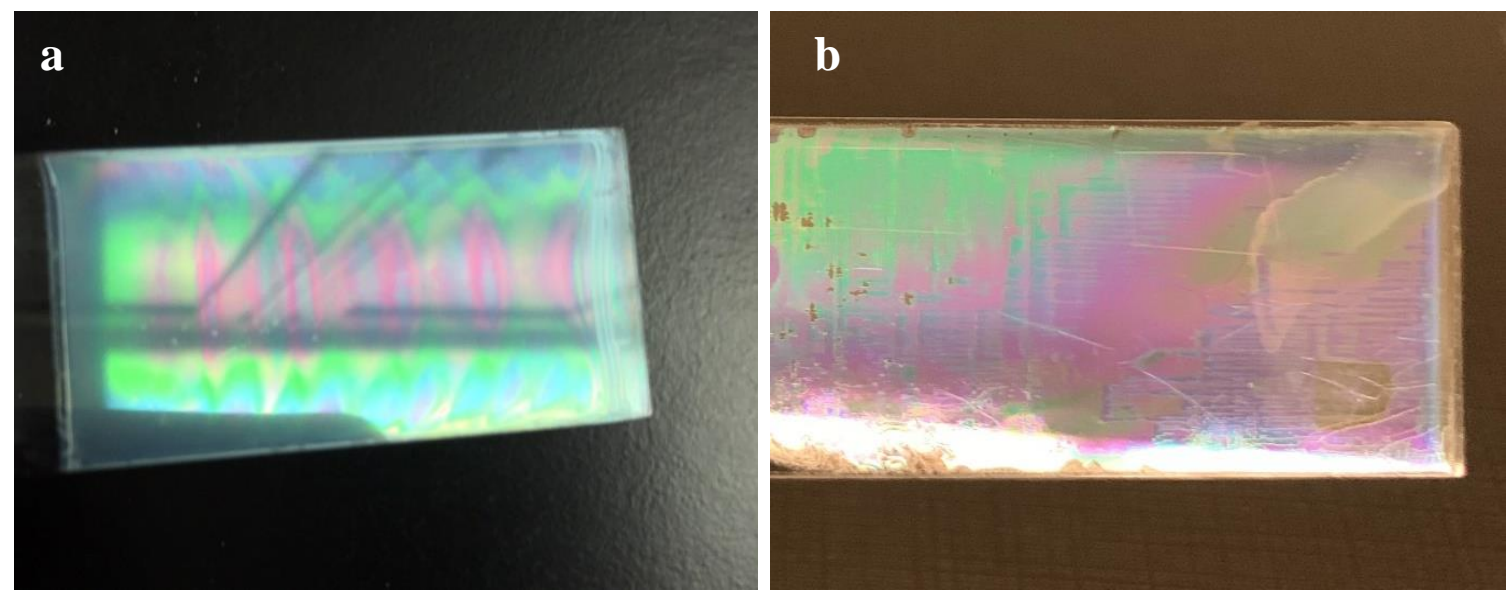

Figure 4.3. Digital photo of the (a) silica colloidal crystal template, and (b) resulting MIP film. 
The sensors were incubated in aqueous solutions cf different concentrations of T. The peak wavelength shift increased with $\mathrm{T}$ concentration, as the example sensor shown in Figure 4.4. When the sensor was incubated in pure water, its spectrum showed a peak at $427 \mathrm{~nm}$ but reshifted $8 \mathrm{~nm}$ in the presence of $1 \mathrm{ppb}$ of $\mathrm{T}$. The diffraction shift of the wavelength gradually increased until it reached $42 \mathrm{~nm},\left(\lambda_{\max }=469 \mathrm{~nm}\right)$ at $10 \mathrm{ppb}$. Figure $4 \mathrm{~b}$ revealed that there is a linear relationship between the Bragg diffraction shift and the $T$ concentrations $\left(R^{2}=0.9817\right)$. The error bars in Figure $4 b$ represents the standard deviation of the three measurements of the same sensor spot at the same concentration.
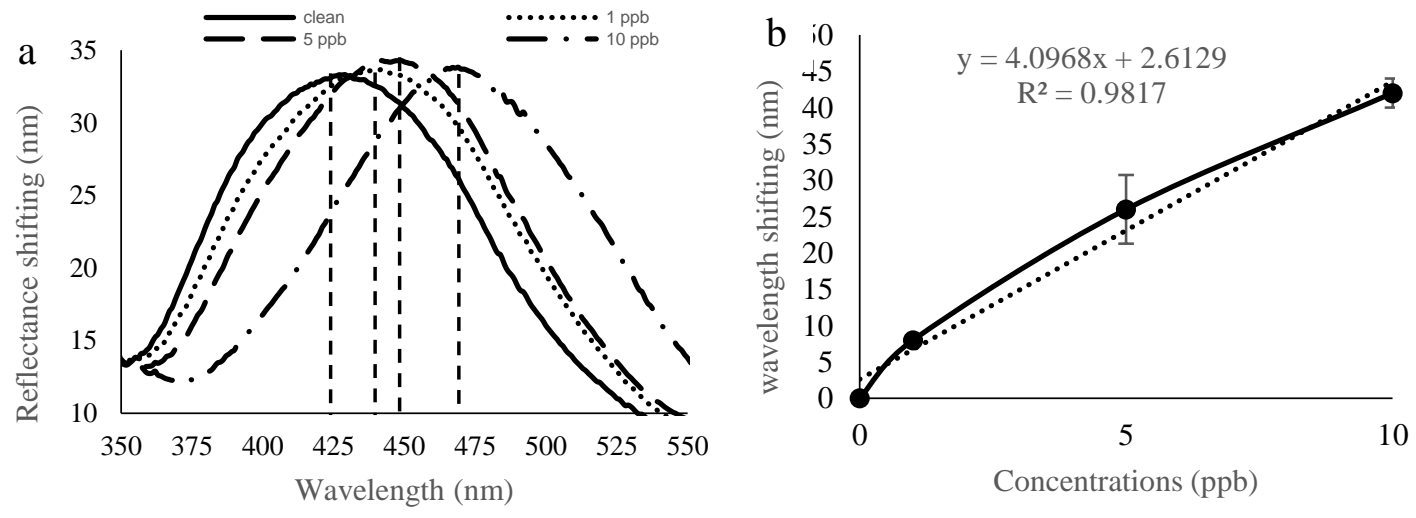

Figure 4.4. Optical properties of sensors: (a) reflectance spectra of MIPs after incubation at various concentrations of $\mathrm{T}$; (b) peak shift for MIPs after incubation at various concentration of $\mathrm{T}$.

Many sensors where fabricated and the standard curve was prepared for each one. However, there are some differences between MIP film led to differences in calibration curves. Figure 4.5 represents the calibration curve for four films that were prepared to use. It is obvious from Figure 4.5 that there is a difference in sensor behavior. The reason for this is that the fabrication of sensors takes several steps, starting from the preparation of colloidal crystals as a template and ending with the washing of the films to remove the target. The completion of these steps takes about 3 days and was done manually by researchers which led to some variability in the colloidal crystal structure, or particles and 
target removal. The results showed that the film was extremely sensitive to any of these variations, and then they can produce significant differences in sensor response.

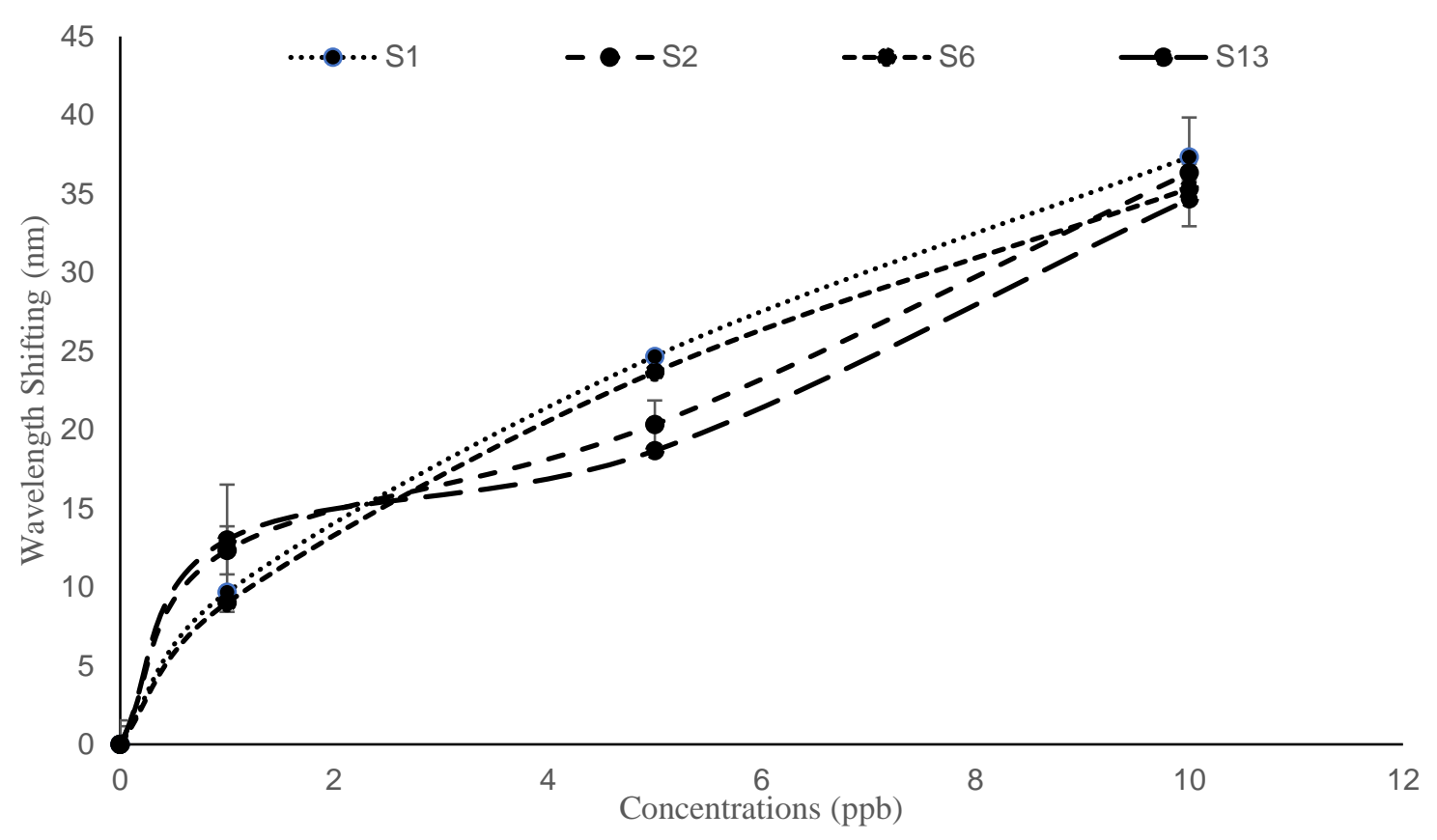

Figure 4.5. The differences in calibration curves for four MIPs film.

\subsubsection{Response time}

The kinetics of the rebinding process was investigated to determine the equilibrium time needed for incubation of the sensor in test samples to measure T. The binding equilibrium time is the time required from the MIP to reach saturation [248]. A quick response to the detection of the $\mathrm{T}$ is an important factor in the sensor design. The ratio of target/ monomer/ cross linker played a key role in this short response time as a comparison with previous work [240]. The sensors were incubated in two different concentrations of 1 $\mathrm{ppb}$ and $5 \mathrm{ppb}$ to investigate the film behavior with different concentrations, and the diffraction peak wavelength was measured at 1, 2, 3, 5, 7, 10, and 15 min. The results showed that an increasing number of T molecules were bound on the MIP with increasing incubation time. However, the length of the shift reached a plateau after 2 min for both concentrations, Figure ( 4.6 a $\&$ b). Therefore, 2 min was chosen as the optimal incubation time. 

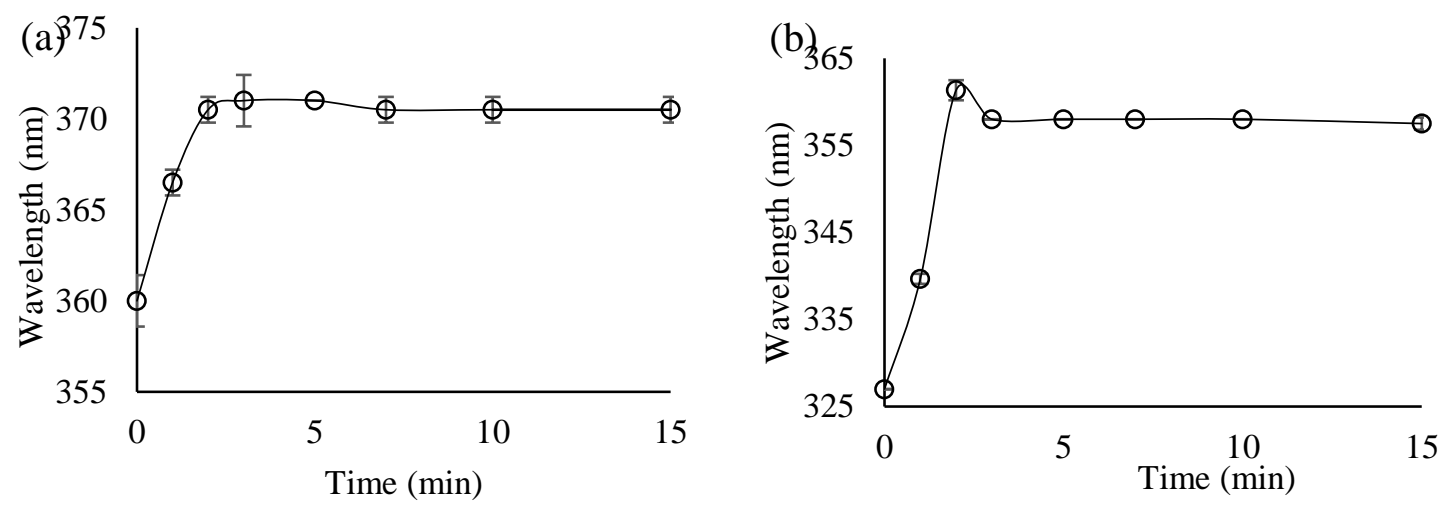

Figure 4.6. Kinetics of MIP response, (a) incubated in $1 \mathrm{ppb}$ of $\mathrm{T}$, (b) incubated in $5 \mathrm{ppb}$ of T

\subsubsection{Investigation of possible interferences in blood samples}

To mimic interferences that can occur in blood, the sensor was incubated in solutions of six steroid hormones at different concentrations and background concentration of $5 \mathrm{ppb} \mathrm{T}$ and $150 \mathrm{mM}$ of $\mathrm{NaCl}$.

Figures 4.7, 4.8, and 4.9 display the experimental results for the $0.04 \mathrm{ppb}, 5 \mathrm{ppb}$, and $30 \mathrm{ppb}$ concentration of the hormones. The results showed that almost all the chemicals had a negligible effect on the T binding at a lower concentration, $0.04 \mathrm{ppb}$, except for DHT and $\mathrm{E}_{2}$. However, when the interferences were present at concentrations of $5 \mathrm{ppb}$ and 30 ppb, the effect of the shift was noticeable for almost all the hormones. Table 4.1 represents the interferences hormones and the expected \% error. The concentrations of the hormones in blood are ranged between 2.29- $20.7 \mathrm{ppb}$ for T [249], 0.11-0.95 ppb for DHT [250], 2.313 ppb for DHEA [251], 0.01-0.29 ppb Cortisol [252], 0.55-72.8 ppb for DHEAS [252], 0.01-0.04 ppb for $\mathrm{E}_{2}$ [253], and 0.02- $4.46 \mathrm{ppb}$ ANDROS [254].

At lower samples concentrations, the DHT and $\mathrm{E}_{2}$ have high percent error 200 \& $240 \%$ which is expected to affect on the testosterone reading, and to get an overestimate value of testosterone if the results for samples test (water samples) in the lab are the same 
behavior of blood samples. The amount of DHT in serum is 0.11-0.95 ppb which mean according to our lab interferences, the concentration of $0.04 \mathrm{ppb}$ would affect the testosterone reading, however, the DHT has the affinity to bind with the sex hormone binding globulin (SHBG) 5 times than T [255], and $99 \%$ of the DHT has the affinity to bind to the protein, where $49.7 \%$ is bounded to SHBG, $0.22 \%$ to the corticosteroid-binding globulin (CBG), and 39.2\% to the albumin [256], which may reduce the effect of the free DHT on T reading.

The amount of E2 in the serum of adult males is 0.01-0.04 ppb, the most amount of E2 are bounded to protein 97\% [257], and the remaining percentage considered the bioactive fraction which can bind to nuclear steroid receptor.

According to the range of DHEA in serum which is $2.3-13 \mathrm{ppb}$, the effected concentration was the $5 \mathrm{ppb}$ which was effect on the T binding by $280 \%$. The DHEA helps to produce hormones like testosterone. The DHEAS did not affect testosterone concentration in both 0.04 and 5ppb, however, at higher concentration, $30 \mathrm{ppb}$, it increased the binding by $60 \%$. Normally, If the level of DHEAS reaches $60 \mathrm{ppb}$ in human body maybe it can refer to the androgen-secreting adrenal tumor [258].

The amount of Co and Andros in serum blood is within the lower concentration of experiments, $0.04 \mathrm{ppb}$. The results showed that there was no significant effect of lower concentration of Co and Andros on the T binding.

One of the causes of other hormones affinity to the T MIP film was the similarities in chemical structure of some hormones to the testosterone, that made facilitate the binding to the testosterone-specific sensor cavities. Besides, the functional groups seemed to affect 
the binding also. For example, DHT had a higher affinity to the T binding sites because of a similarity in functional groups. In contrast, DHEAS which has a different functional group, the sulfate group, showed little to no effect at the investigated concentrations.

Since one sensor was used for all hormones, there could also be some effects of diminished efficiency as MIPs were washed, regenerated, and used again which may contradict the results from previous work [240]. However, the testosterone solution was saline at a concentration of $150 \mathrm{nM}$ this time, and the washing method was changed by adding a stirring method to remove the testosterone which seems to affect the rigidity of film and influence the efficiency of regenerating sensor. Given the low cost of the sensor, this observation does not constitute a real problem since if converted into a commercial product, the sensor would be recommended to be one use only, which is also recommended given their use in blood samples, from a health point of view.

Table 4.1. Interference of selected hormones on the assessment of a $5 \mathrm{ppb} \mathrm{T}$ solution by MIP sensor

\begin{tabular}{|l|l|l|l|l|}
\hline \multirow{2}{*}{ Interference } & $\begin{array}{c}\text { Concentration } \\
(\mathrm{ppb})\end{array}$ & $\begin{array}{c}\text { Wavelength } \\
\text { shift }(\mathrm{nm})\end{array}$ & $\begin{array}{c}\text { Apparent T } \\
\text { concentration }\end{array}$ & \% Error \\
\hline \multirow{5}{*}{ DHT } & 0.04 & 25 & 15 & 200 \\
\cline { 2 - 5 } & 5 & 36 & 32 & 540 \\
\cline { 2 - 5 } & 30 & 36 & 32 & 540 \\
\hline DHEA & 0.04 & 18 & 5 & 0 \\
\cline { 2 - 6 } & 5 & 27 & 19 & 280 \\
\cline { 2 - 6 } & 30 & 51 & 46 & 820 \\
\hline \multirow{3}{*}{ CO } & 0.04 & 19 & 6 & 20 \\
\cline { 2 - 6 } & 5 & 21 & 9 & 80 \\
\cline { 2 - 6 } & 30 & 23 & 12 & 140 \\
\hline \multirow{2}{*}{ DHEAS } & 0.04 & 18 & 5 & 0 \\
\hline
\end{tabular}




\begin{tabular}{|l|l|l|l|l|}
\hline \multirow{2}{*}{} & 5 & 18 & 5 & 0 \\
\cline { 2 - 5 } & 30 & 20 & 8 & 60 \\
\hline \multirow{3}{*}{ E2 } & 0.04 & 26 & 17 & 240 \\
\cline { 2 - 5 } & 5 & 27 & 19 & 280 \\
\cline { 2 - 5 } & 30 & 28 & 20 & 300 \\
\hline \multirow{3}{*}{ ANDROS } & 0.04 & 19 & 6 & 20 \\
\cline { 2 - 5 } & 5 & 29 & 22 & 340 \\
\cline { 2 - 5 } & 30 & 30 & 24 & 380 \\
\hline
\end{tabular}

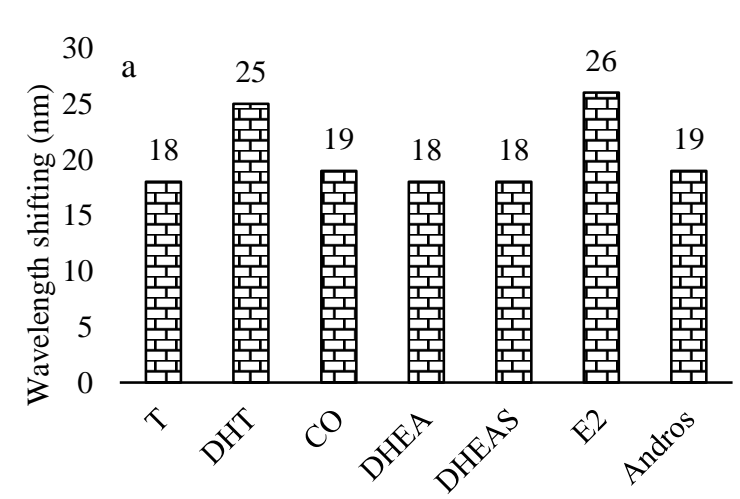

Hormones

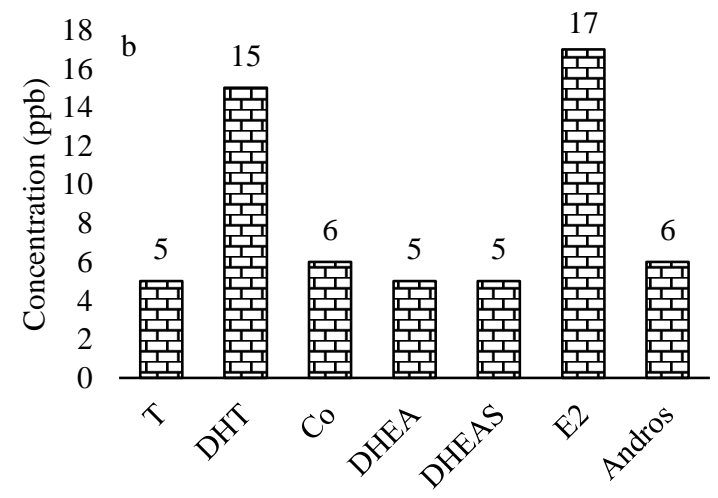

Hormones

Figure 4.7. MIP sensor response incubated in a $5 \mathrm{ppb} T$ solution, in the presence of $0.04 \mathrm{ppb}$ of interfering hormones and $150 \mathrm{mM} \mathrm{NaCl}$, (a) wavelength shift (b) sensor T measured concentrations, calculated from wavelength shift and calibration curve. First bar corresponds to the control $5 \mathrm{ppb}$ T solution in the absence of interferences.
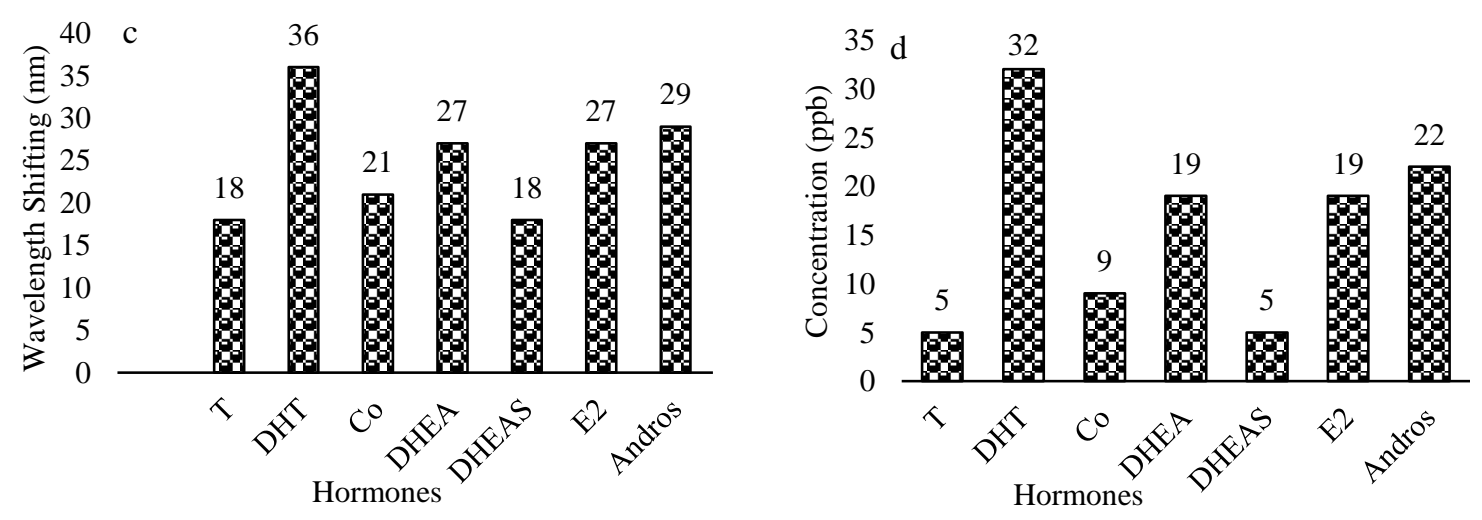
Figure 4.8. MIP sensor response incubated in a $5 \mathrm{ppb} \mathrm{T}$ solution, in the presence of 5 ppb of interfering hormones and $150 \mathrm{mM} \mathrm{NaCl}$, (a) wavelength shift (b) sensor T measured concentrations, calculated from wavelength shift and calibration curve. First bar corresponds to the control $5 \mathrm{ppb} \mathrm{T}$ solution in the absence of interferences.
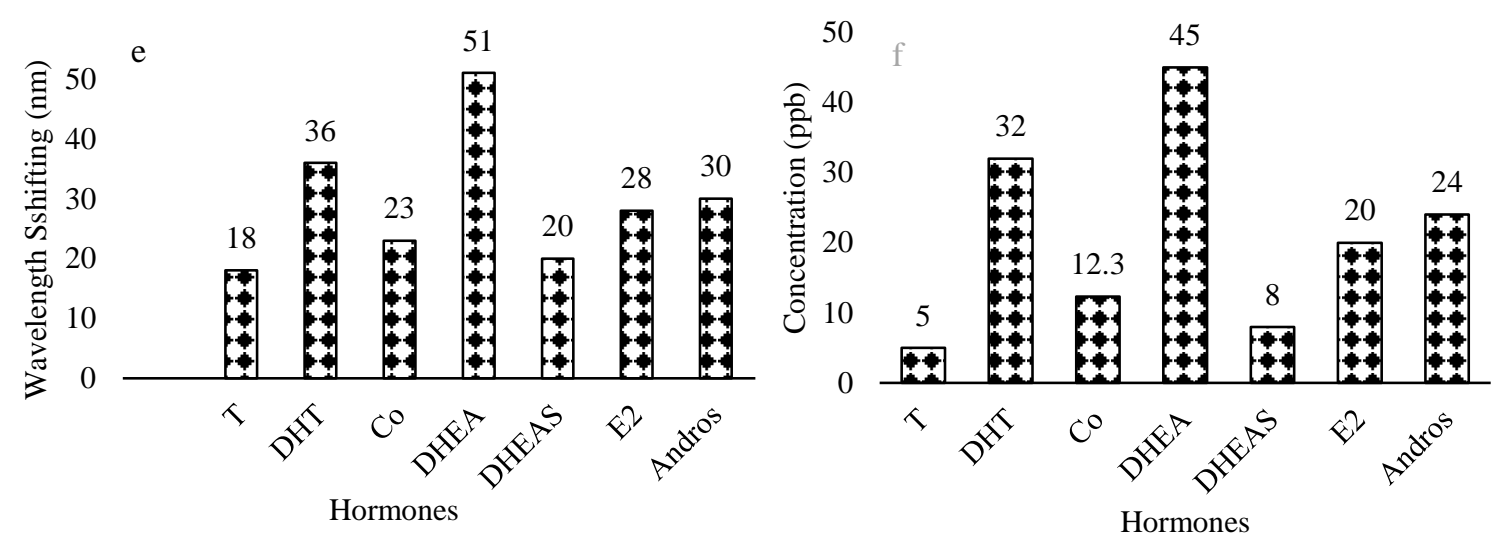

Figure 4.9. MIP sensor response incubated in a $5 \mathrm{ppb} \mathrm{T}$ solution, in the presence of $30 \mathrm{ppb}$ of interfering hormones and $150 \mathrm{mM} \mathrm{NaCl}$, (a) wavelength shift (b) sensor T measured concentrations, calculated from wavelength shift and calibration curve. The first bar corresponds to the control $5 \mathrm{ppb}$ T solution in the absence of interferences.

The affinity of the selected hormones to the testosterone binding sites is related to the 3D structure of the molecule and their functional groups. This relationship was investigated by molecular modeling. The molecular electrostatic potential surface (MESP), which is a plot of electrostatic potential mapped onto the isoelectronic density surface simultaneously displays molecular shape, size, and electrostatic potential values are very useful in the investigation of the molecular structure with its physicochemical property relationships [259]. Figure 4.10 shows the top view and Figure 4.11 shows the side view of the MESPs for $\mathrm{T}$ and the investigated hormones. Regions with the most negative and most positive electrostatic surface potentials are colored with red and blue, respectively. From the figures, it can be seen that the DHEAS and CO have a larger size than testosterone 
due to the sulfate group and the alpha-hydroxy ketone group respectively. That difference can explain the result obtained during interference studies, where almost no-interference effect has been observed with those hormones even at high concentration (Figure 4.9).

The fact that the other hormones have a size that is similar to $\mathrm{T}$ explains the interference observed during experiments at an equal concentration of $\mathrm{T}$ and interference (Figure 4.8). Among all the interferences tested, only the DHT and E2 showed effects at the lowest concentration (Figure 4.7). This can be explained considering the electrostatic potential. $\mathrm{T}$ has a region with negative potential on the first six-member ring due to the oxygen of the carbonyl group and a region of positive potential on the five-member ring due to the hydrogen of the hydroxyl group; exactly the same distribution of electrostatic potential was found for DHT.

While $E_{2}$ exhibits similarities in the distribution of electrostatic potential, the molecule has an extra positive potential on the first six-member ring due to the hydrogen of the hydroxyl group. However, that hydroxyl group is bonded to an aromatic ring and, in consequence, that part of the molecule is planar (Figure 4.11) and then smaller than the testosterone and may not constitute a significant steric hindrance. 


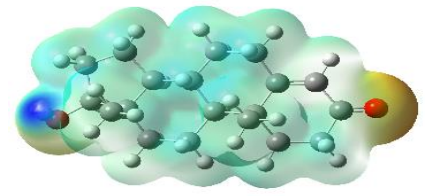

$\mathrm{T}$

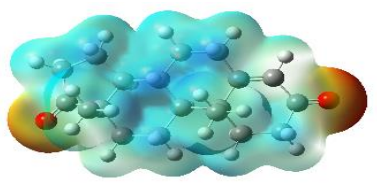

Andros

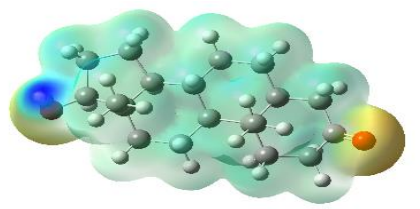

DHT

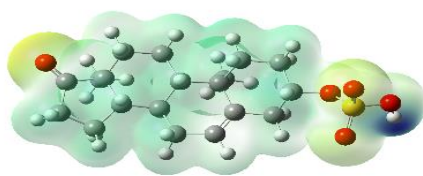

DHAES

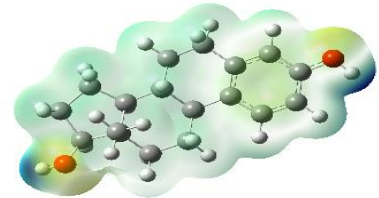

$\mathrm{E}_{2}$

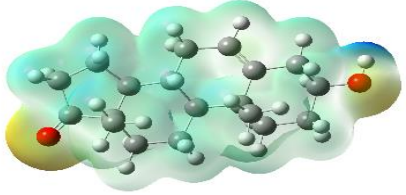

DHAE

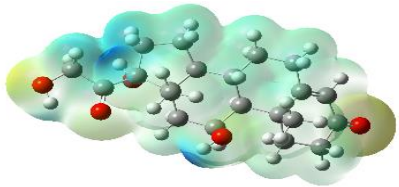

Co

Figure 4.10. Top view of the molecular electrostatic potential surfaces (MESPs) for the interfering hormones. Regions with most negative and positive electrostatic surface potentials are colored with red and blue, respectively.

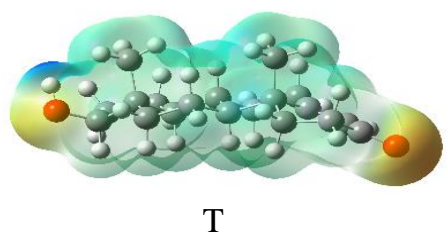

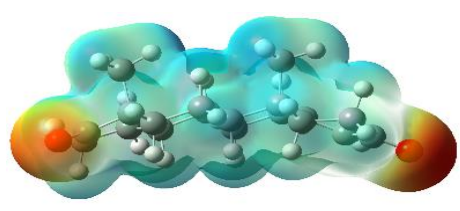

Andros

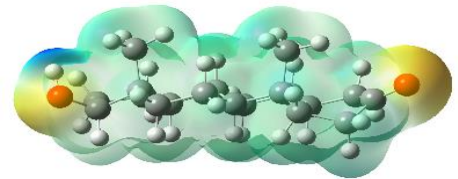

DHT

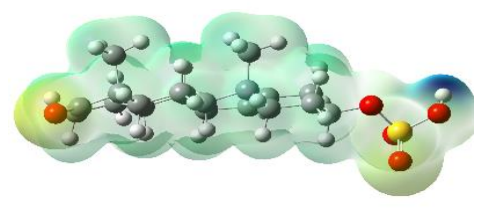

DHAES

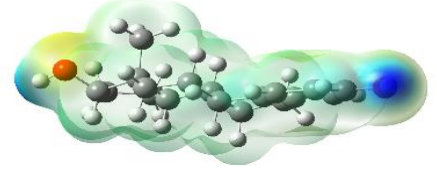

$\mathrm{E}_{2}$

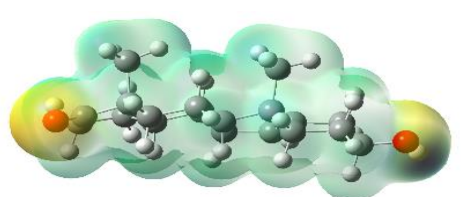

DHAE

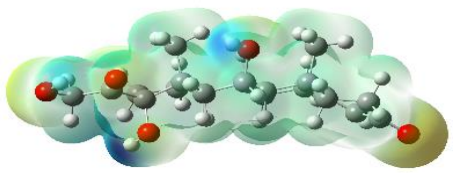

Co

Figure 4.11. Side view of the molecular electrostatic potential surfaces (MESPs) for the interfering hormones. Regions with most negative and most positive electrostatic surface potentials are colored with red and blue, respectively. 


\subsubsection{Analysis of blood samples}

To explore the practical application of the $\mathrm{T}$ sensor, the sensor was incubated in horse whole blood and human plasma samples. Original samples were spiked with $\mathrm{T}$ solution to increase the range of $\mathrm{T}$ concentration to be measured by the sensor. Figure 4.12 shows the optical response of the sensor to the unspiked and spiked horse blood samples with $\mathrm{T}$. An increase of the peak wavelength shift with the increase of $\mathrm{T}$ concentration was observed. The sensor was incubated on blood samples without pretreatment for 2 mins, and then the sensor was washed by ultrapure water and tested under UV-spectrophotometer. The results showed that there was a direct proportional relationship between the concentrations and shift wavelength which means that the sensor can be used in blood successfully without pretreatment. This gives the advantage of the sensor to be used at the patients' home, or as a point of care device, as the physician office.
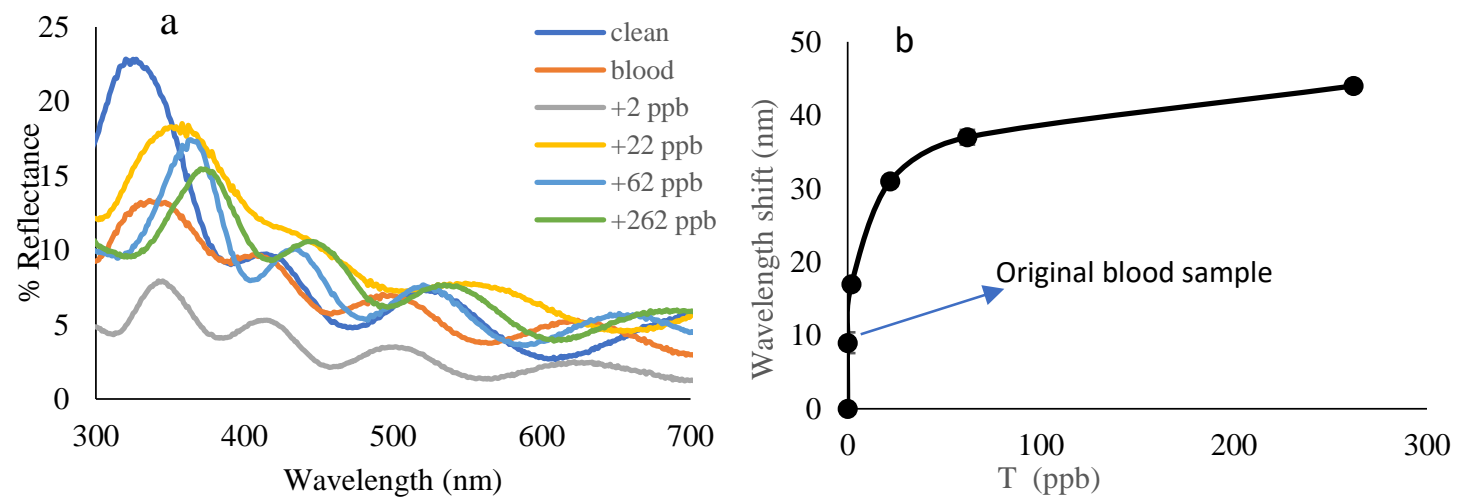

Figure 4.12. Application of sensors on a horse blood samples: (a) reflectance spectra of sensor after incubation at horse blood samples spiked with various concentrations of T; (b) diffraction peak shift of sensor after incubation at horse blood samples spiked with various concentrations of $\mathrm{T}$.

Human blood samples from an unidentified donor were used to assess the efficiency of sensors to detect the level of free or total $\mathrm{T}$ in human blood. Two sensors were used to measure each sample. The samples were divided into unspiked and spiked with $100 \mu \mathrm{L}$ of 1,10 , and $30 \mathrm{ppb}$ of $\mathrm{T}$. Aliquots of unspiked and spiked samples were sent to a clinical 
laboratory for validation. The sensors were regenerated after each sample measurement and incubated again to investigate the reuse of the photonic sensor. Table 4.2 represents the number of sensors and the results from and the values of free and total $\mathrm{T}$ from the clinical laboratory.

Sensors 1 and 2 were used to measure the level of $\mathrm{T}$ in the unspiked, as collected sample. While the clinical lab determines the concentration of $\mathrm{T}$ to be $0.074 \mathrm{ppb}$, the values reported by the sensor were much higher, at $5.9 \mathrm{ppb}$ and $5.4 \mathrm{ppb}$ for sensor 1 and sensor 2 respectively. The hypothesized reason for that was that the sensor did not just detect the free testosterone but potentially also bound testosterone, resulting in the measurements of the total testosterone.

Sensors 3 and 4 were applied to the analysis of the spiked sample with $1 \mathrm{ppb}$ of testosterone. The results for the spiked samples showed that the level of $\mathrm{T}$ was $0.103 \mathrm{ppb}$ at M.C.Whereas, the values reported by the sensors were $10.3 \mathrm{ppb}$ and $19.6 \mathrm{ppb}$. The results of the sensors were again higher than the laboratory testing, which further supports our hypothesis that the sensor may detect part of the total testosterone. The result of clinical laboratory for the samples spiked with $10 \mathrm{ppb}$ showed that the results of free testosterone were $0.733 \mathrm{ppb}$, however, the results for sensors 5 and 6 showed the readings of $\mathrm{T}$ were $0.4 \mathrm{ppb}$ and $4.5 \mathrm{ppb}$. The Lab reading of testosterone showed that these results also not within the lower detection of our sensor ( $1 \mathrm{ppb})$, however, sensor 5 showed a reading with $42 \%$ error.

After spiked the samples with $30 \mathrm{ppb}$, the M.C. results showed that the reading was $3.4 \mathrm{ppb}$ of free testosterone which is within the concentrations that the sensor can 
detect. However, the reading of sensors 7 and 8 showed that the reading was 4.8 and 4.4 ppb which represents $41 \%$ and $29 \%$ of reading error respectively.

The samples without testosterone added and with 1 and 10 ppb's addition tested at the clinical laboratory, showed that the values of free testosterone were less than the minimum value that the sensor can detect (1ppb). However, the reading of the sensors showed high values for lower concentrations. The reason for that can be clarified from a different perspective: first, the interferences of the other hormones which showed that the free testosterone will be increased extremely even with a very low concentration of hormones interferences $(0.04 \mathrm{ppb})$. Second, the higher amount of compound testosterone, table 4.2 , appeared when the samples were spiked which may be either detect directly by sensor or release and transfer to free testosterone and increased the reading. In either way, this was a preliminary test to detect free testosterone by optical sensors, and it showed that more tests have to implemented to prove the efficiency of the sensor and overcome the problems that faced, as well as an assessment of the levels of other hormones in blood to better interpret the results.

The results for the reused sensor after regenerating showed that all the sensors for unspiked and spiked samples were not in good agreement which means that the sensor cannot be reused for the blood tests. This was may be due to damaging of testosterone binding site inside MIP during washing the sensor to regenerate again, or non-specific binding of other compounds found in the blood. It is clear from the results in Table 4.2 that sensors were not able to re-use after $3^{\text {rd }}$ regenerated because of the damage of the sensors that result from re-washing which maybe cause the sites to damage. However, even with 
the $1^{\text {st }}$ reuse of all the sensors, the values changed clearly which means that the sensor can be used just one time.

Table 4.2 $\mathrm{T}$ analysis in blood samples by photonic sensor and reference lab.

\begin{tabular}{|l|l|l|l|l|l|l|l|}
\hline Sample & Sensor \# & Meas.1 & Reuse 1 & Reuse 2 & $\begin{array}{l}\text { Clinical } \\
\text { lab. Free T } \\
(\mathrm{ppb})\end{array}$ & $\begin{array}{l}\text { Clinical lab } \\
\text { Total T } \\
\text { (ppb) }\end{array}$ & Error * \\
\hline As collected & 1 & 5.9 & 3.8 & N/A & 0.0714 & 2.55 & $\mathbf{8 1 6 3}$ \\
\hline & 2 & 5.4 & 3.8 & N/A & 0.0714 & 2.55 & $\mathbf{7 4 6 3}$ \\
\hline Spiked 1ppb & 3 & 19.6 & 15.8 & 5.9 & 0.103 & 3.56 & $\mathbf{1 8 9 2 9}$ \\
\hline & 4 & 10.3 & 13.6 & 9.3 & 0.103 & 3.56 & $\mathbf{9 9 9 7}$ \\
\hline Spiked 10 ppb & 5 & 0.4 & 10.8 & N/A & 0.733 & 19.8 & $\mathbf{4 5}$ \\
\hline & 6 & 5.2 & 7.1 & N/A & 0.733 & 19.8 & $\mathbf{6 0 9}$ \\
\hline Spiked 30 ppb & 7 & 4.8 & 6.9 & 2.3 & 3.4 & 67.9 & 41 \\
\hline & 8 & 4.4 & N/A & N/A & 3.4 & 67.9 & 29 \\
\hline
\end{tabular}

computed for Meas.1, which corresponds to an unused MIP film.

\subsection{Conclusions}

In this work, a novel technique based on photonic crystal and molecularly imprinted polymer was evaluated for the analysis of testosterone in blood. The MIPs may provide an easy and fast technique to detect the $\mathrm{T}$ in human blood; however, the sensors were affected by interferences with the other hormones and the results showed great variation when compared to the concentrations obtained from a clinical laboratory.

The modeling showed that the hormones that have a similar structure and functional group affected testosterone detection even with a lower concentration, however, the hormones that have a big structure as a result of the functional group had a lower or no effect on the testosterone detection.

If limitations evidenced by this work could be overcome, this type of sensor may provide a highly needed fast, inexpensive and easy to use a diagnostic tool to detect the $\mathrm{T}$ by integrated with technology such as a smartphone for telemedicine applications. 
It is clear from table 4.2 that the total testosterone was within the detection range even for the unspiked samples. Future research may include tests by adding sodium acetate to the blood samples to release all the bounded testosterone and convert it to free $\mathrm{T}$. In this case, the sensor will be used to measure the total testosterone in the blood. 


\section{Chapter 5}

\section{Using the Molecularly Imprinted Polymer Technique to Screen for Endocrine Disrupting Chemicals Activity}

\subsection{Introduction}

Endocrine-disrupting chemicals (EDC) are substances that interfere with the endocrine system in humans and wildlife and lead to different developmental, reproductive, neurological, and immune problems[260][8]. Some of these chemicals like estradiol and testosterone are natural while others are synthetic such as pharmaceuticals, dioxin, and dioxin-like compounds, pesticides, and plasticizers[5]. EDCs can influence the endocrine system totally or partially by mimicking the natural hormones and binding to the receptors inside the cell blocking the receptor, they can stimulate (agonists) or inhibit (antagonists) these receptors [261].

To determine EDC's activity, the most effective methods are biologically based assays which are useful in studying the effects of EDCs and the identification of compounds that could possibly be EDCs. Several mechanisms are tested in these assays such as ligand binding, cell proliferation, or antigen-antibody interactions[262]. Ligand binding can quantify the number of specific binding sites, whereas cell-binding uses the estimation for cell growth and reproduction. Antigen-antibody interactions use noncovalent binding of antigen to antibodies. These assays can be applied with whole organisms like birds and fish or cellular and non-cellular material such as antibodies or androgen receptors. Mass spectroscopy-based analytical methods provide excellent sensitivity and precision to quantify EDCs, however, they are limited in describing the 
overall estrogenic effects, such as the synergistic or anti-estrogenic influences in the presence of multiple EDCs so they serve for the quantitative analysis of EDCs. In general, mass-based methods are composed of a pretreatment or extraction step followed by instrumental analysis. Various types of instruments were used such as HPLC-MS/MS [263], LC-MS/MS [6], GC-MS/MS[264].

The biological based methods suffer from some challenges in detection and analysis procedures derived from the trace concentration levels and extremely diverse group of EDCs. As a result, several methods that were used to analyze EDCs incorporated also mass-based methods such as E-screen [265], estrogen responsive chemically activated luciferase expression (ER-CALUX)[266], yeast estrogen screen (YES)[267], and enzymelinked immunosorbent assay (ELISA)[268] which make them complicated and required more operator training.

We have recently reported the fabrication of a photonic sensor for the detection of testosterone in aqueous samples. The sensor combines a molecularly imprinted polymer (MIP), which provides specificity, and an inverse opal morphology that is photoactive and reacts to the level of testosterone that is bound to the MIP. The photonic sensor is a modern technique to detect different contaminants in a fast and straightforward method. MIPs have been referred to as artificial receptors, and therefore it is prepared as an alternative way to detect EDCs in water mimicking their natural counterparts[240].

To provide a fast and straightforward method to screen the EDCs as a comparison with the traditional method, we investigated the application of the photonic sensor as an androgenic EDC screening method. The approach consisted of determining which chemicals that are considered as EDCs, can bind to the testosterone-MIP and how their 
binding affinity compares to the actual hormone. The objective of this work is to evaluate if and which endocrine disrupting chemicals interact with the testosterone sensor in a similar way as testosterone, as a first screening method for androgenic ED activity.

\subsection{Materials and Methods}

\subsubsection{Materials}

All chemicals were purchased from Sigma-Aldrich (St. Louis, USA) and used without further purification; ethanol (200 proof, ACS reagent, 99.5\%) was used for the preparation of the dispersion of silica particles, acrylic acid (AA) (99\%), ethylene glycol dimethacrylate (EGDMA) (98\%), 2,2'-azobisisobutyronitrile (AIBN) (98\%), and testosterone (T1500) were applied in making molecularly imprinted polymer prepolymerization solution. Hydrofluoric acid (HF) (49\%) was used to remove the silica particles. Tested endocrine disrupting chemicals included: Bisphenol A (BPA), Flutamide (FLU), Estradiol (EST), Dichlorophene (DCP), 1,1,1-Trichloro-2,2-bis(4chlorophenyl)ethane (p,p'-DDT), Dichlorodiphenyldichloroethylene (p,p'-DDE), Chlorpyriphos (CHL), and Vinclozolin (VIN). Commercial silica particles with a diameter of $300 \mathrm{~nm}$ were purchased from Pinfire - Gems \& Colloids (Frankfurt, Germany). Glass microscope slides with dimensions $1 \times 13 \times 76 \mathrm{~mm}$ were obtained from Fisher Scientific (Pittsburgh, PA, USA). Poly methyl methacrylate (PMMA) slides were purchased from ePlastic (San Diego, CA, USA) and cut into the same dimension of glass slides. Ultra-pure water $\left(18.2 \mathrm{M} \Omega \cdot \mathrm{cm}\right.$ at $\left.25{ }^{\circ} \mathrm{C}\right)$ was used in all experiments and obtained from a Thermo Scientific ${ }^{\mathrm{TM}}$ Barnstead $^{\mathrm{TM}}$ E-Pure ${ }^{\mathrm{TM}}$ Ultrapure Water Purification System (Waltham, MA, USA). 


\subsubsection{Methods}

\subsubsection{Preparation of silica particle colloidal crystals}

The colloidal crystals were fabricated following the same procedure described in a previous publication [240], Briefly, colloidal crystals made of silica particles were obtained by self-assembly [84] First, suspensions were prepared by adding 148.5 milligrams of commercial silica particles to $75 \mathrm{~mL}$ of ethanol in a flask and stirring at $300 \mathrm{rpm}$ for $1 \mathrm{hr}$ and then sonicating for $1 \mathrm{hr}$. The stirring and sonication were repeated 5 times respectively. Then, a cleaned glasses slide with a $1 \times 13 \times 76 \mathrm{~mm}$ were vertically placed in a $20 \mathrm{ml}$ of suspension silica particles in ethanol (volume fraction $0.1 \%$ ) in a furnace for $24 \mathrm{hr}$ at a temperature of $50^{\circ} \mathrm{C}$, ethanol was evaporated to allow a colloidal crystal to form on both sides of the slides.

\subsubsection{Synthesis of molecularly imprinted photonic sensor}

Figure 5-1 shows the procedure for the fabrication of a molecularly imprinted polymer sensor. The formulation of the imprinted polymer consisted of a prepolymerization solution of target/monomer/crosslinker at a molar ratio of 1:32:8, respectively. A mass of $72 \mathrm{mg}$ of the target molecule testosterone was dissolved in $1 \mathrm{ml}$ of ethanol, followed by the addition of 550 $\mu 1$ of AA, $380 \mu 1$ of EGDMA, and $9 \mathrm{mg}$ of AIBN. The compounds were stirred until a homogeneous mixture was obtained and then poured in a small beaker. PMMA slides were placed on both sides of the glasses slide containing the colloidal crystals and the three slides were firmly held together, forming a "sandwich" structure. The lower end of the sandwich structure was dipped in the prepolymerization solution, and it was raised by capillary force into the colloidal crystal, filling the void 
spaces in the silica particle deposit. The assembly was then put under UV light at $365 \mathrm{~nm}$ for $3 \mathrm{hr}$ to complete the polymerization. Then, the structure was immersed into 5\% hydrofluoric acid for $12 \mathrm{hr}$ in order to etch the glass and particles so that the particles are removed, and the three slides separated. After that, the films were washed to remove the testosterone in a 1:9 (v/v) acetic acid: ethanol washing solution for 5 cycles with a clean solution each time. Each cycle consisted of submerging the film in $25 \mathrm{~mL}$ of the washing solution and stirring at $50 \mathrm{rpm}$ for 30 minutes. At the end of each washing step, the solution was disposed and replaced for a fresh one immediately until 5 washes were completed. One last rinse was performed with ethanol only to remove any remaining acid that may damage the polymer.
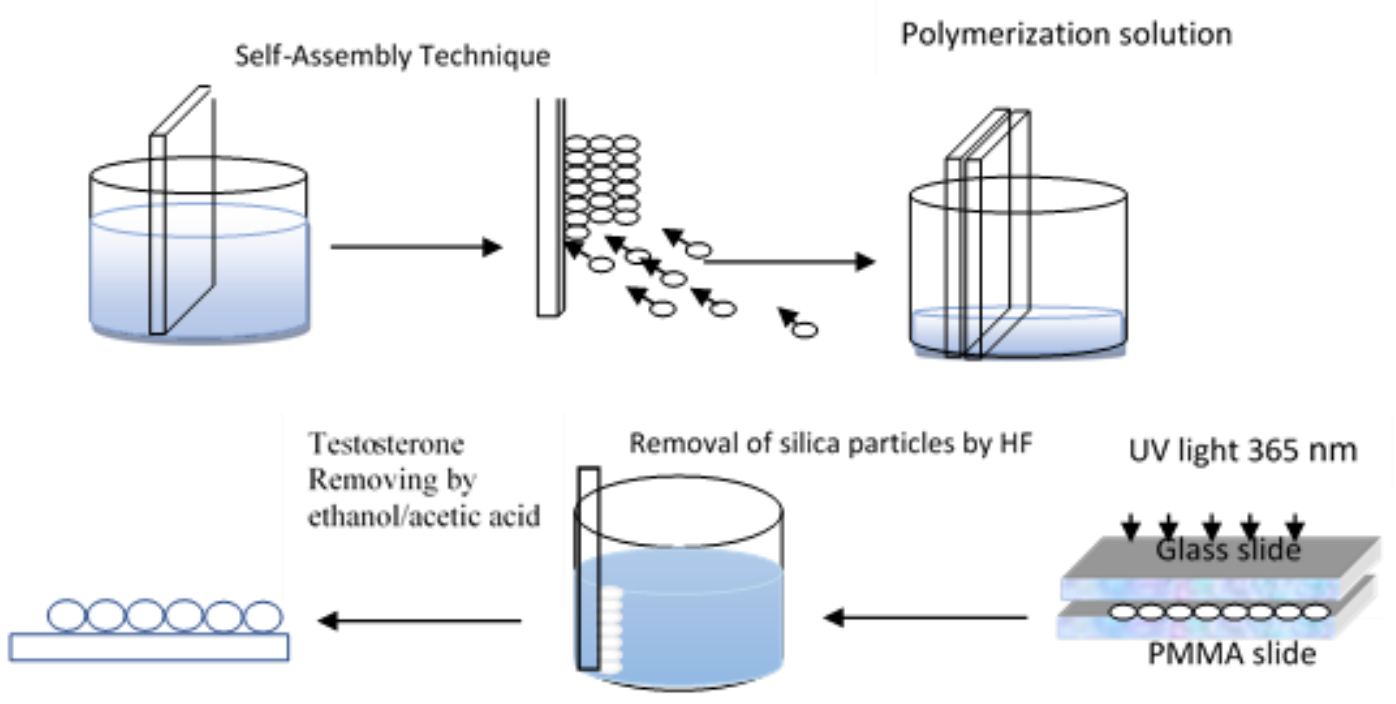

Figure 5.1 Synthesis of molecularly imprinted polymer and detection of EDCs

\subsubsection{Characterization of silica colloidal crystal template and MIPs}

The size of the particles and the morphology of colloidal crystals were studied by an FEI Quanta 600 FEG Environmental Scanning Electron Microscopy (ESEM). The samples were mounted on a stub of metal with adhesive, coated with a $1.5-3 \mathrm{~nm}$ layer of 
gold by using a sputter coater and then observed in the microscope. Then, Esprit 2.1 software (Madison, WI), a software for nano and microanalysis, was used to confirm the size of silica particles. The software was used to analyze the SEM images. The images were filtered, corrected, and processed under the software to confirm the size of silica particles. The fabricated film porous structure was observed by SEM.

\subsubsection{Optical response of MIP sensors}

$\mathrm{UV}-\mathrm{V}$ is reflectance spectra were used to characterize optical response and calibrate the sensor. The reflectance of the photonic hydrogel films was measured over a wavelength range of $200-800 \mathrm{~nm}$, using a double-beam UV-Vis spectrophotometer (Cary 60, Varian) with a Harrick Scientific's Specular Reflection Accessory (ERA-30G) for measurement reflectance at a fixed angle of 30 degrees.

The protocol to record sensor response is the following: the sensors were first incubated in $25 \mathrm{~mL}$ of pure water until fully hydrated and three independent spectra were collected, as the blank. Then, sensors were incubated in the corresponding test solution until equilibrium was attained, rinsed with ultrapure water, and any excess water removed before placing in the spectrophotometer to record the reflectance spectra. The response time for the equilibrium of the sensors was 2 minutes, following work previously reported in chapter four.

\subsubsection{Selection of the Endocrine Disrupting Chemicals (EDCs)}

The chemicals were selected so that they reflect both similar and dissimilar structures to that of the target molecule, testosterone, and given their known ED activity. Some of these chemicals have been shown to interfere with androgen receptors while others with estrogen receptors. The chemicals have different uses pesticide, fungicide, and 
bactericide. Also, there are differences in their toxicity; some of them appeared under the ToxPi index highly toxic, and for other, the toxicity was low. Estradiol (EST) was selected according to its similarity with testosterone to see the interference between the similar structures. Bisphenol A (BPH) affects the AR receptor by acting as an androgen antagonist which leads to inhibition of $\mathrm{T}$ binding [269]. Flutamide (FLU) is a hormone based chemotherapy. It can prevent prostate cancer. It is a competitive antagonist of the androgen receptor, competing with androgens like testosterone for binding to ARs [270]. DDE is an androgen receptor antagonist [271]. DDT has a hormone-like activity and can bind to the estrogen receptor. DDT, DDE, and BPH derivatives have structural similarities in their chemical features, and they act as an antagonist to androgen or estrogen receptors [272]. Vinclozoline (VIN) is a fungicide, which competes with natural androgen and blocks it [273]. Chlorpyrifos (CHL) is an antiandrogen pesticide that competes with the hormones of the human androgen receptor [274]. Also, dichlorophen (DCP) is a bactericide and fungicide, used in the treatment of tapeworm and cosmetics for humans, and it is an antiandrogen chemical [275]. Figure 5.2 showed the structures of those eight endocrine disrupting chemicals and the target of the sensor (testosterone).

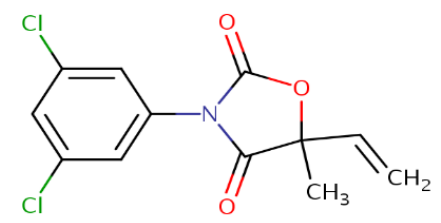

vinclozolin

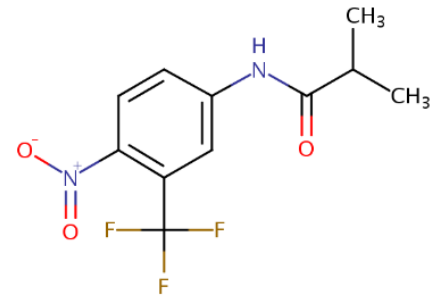

flutamide<smiles>Oc1ccc(Cl)cc1Cc1cc(Cl)ccc1O</smiles>

dichlorophen 


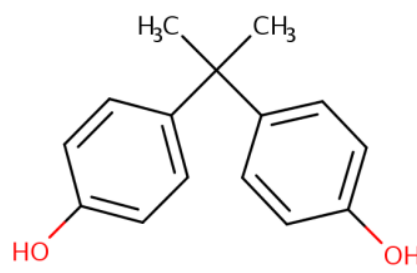

bisphenol A

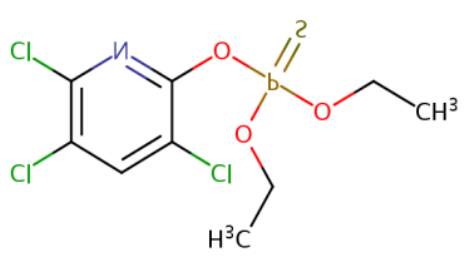

chlorpyrifos<smiles>ClC(Cl)=C(c1ccc(Cl)cc1)c1ccc(Cl)cc1</smiles><smiles>Clc1ccc(C(c2ccc(Cl)cc2)C(Cl)(Cl)Cl)cc1</smiles>

$\mathrm{p}, \mathrm{p}^{\prime} \mathrm{DDE}$
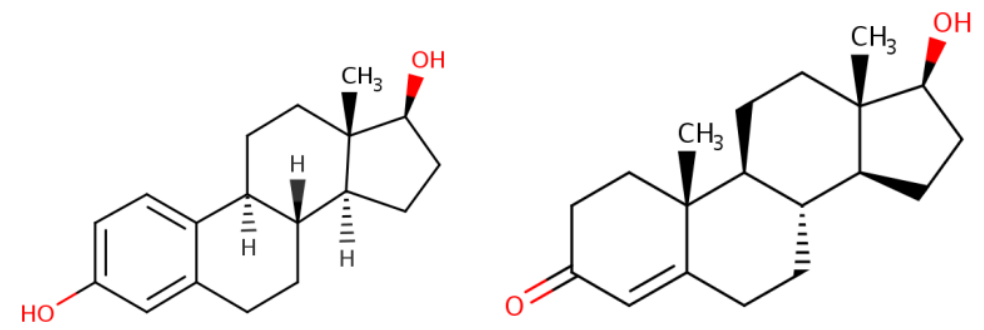

testosterone

Figure 5.2 Structures of the testosterone and eight of the endocrine disrupting chemicals

\subsubsection{Toxicological Prioritization Index software (ToxPi)}

Toxicological prioritization index software (ToxPi) was used to explain the toxicity of chemicals that were studied in this chapter. The software has a dimensionless index score that is calculated for each EDCs as a weighted combination of all the data sources that represent three different domains [276]. Three different database websites were used to get the data used by ToxPi: ECOTOX database, Toxicity Forecaster (ToxCast), and the conditional toxicity value (CTV) predictor. ToxPi represents as a component slides, each slide representing one parameter. Each component is given specific weight from total weight according to its importance and the researcher's goal. In this chapter, the components were given equal weight (25\%) because this research focuses on 
the general comparison between chemicals according to their toxicity. Four parameters were selected from the three data sources to calculate the index score which are AC50, reference dose toxicity (RfD), cancer potency value, and average lower concentration. Any component data that were missing was assumed to be zero.

The ECOTOX database is a source for in vivo toxicity data for aquatic life, terrestrial plants, and wildlife that was developed by the U.S. EPA's National Health and Environmental Effects Research Laboratory's Mid-Continent Ecology Division [277]. The main source for these data is peer-reviewed literature. The chemicals were identified by their CAS number. EC50 for the aquatic organism was chosen here as an endpoint, and the lowest toxic concentration for the aquatic life was chosen to apply in ToxPi software.

The US Environmental Protection Agency (EPA's) Toxicity Forecaster (ToxCast) is a test designed to perform chemical prioritization, hazard identification, and risk assessment [278]. It contains a screen library, dealing with in vitro assay toxicity data. The ToxCast has data for about 1800 chemicals from a variety of sources such as industrial, food, and consumer products. The AC50 was selected from this database to use as one parameter in ToxPi software. The AC50 represents the concentration of a chemical that gives a half-maximal response. The value of AC50 was obtained from the values of NovaScreen panel (NVS) nuclear receptor assay for humans (NVS_NR_hAR). However, if this value was not available, the androgen receptor for Chimpanzee (NVS_NR_cAR), or Bovine was used (NVS_NR_bAR). The AC50 was calculated under three models, which are constant model at zero, gain-loss model, and hill model. After fitting each model, the model with the best fit curve was selected, and the AC50 was calculated from the intersection to obtain concentration that gives 50\% activity for that chemical. 
The conditional toxicity value (CTV) predictor is a model based on the in silico approach which is able to generate a prediction for human toxicity based on chemical properties [279]. It can be used to predict toxicity values such as point of departure, a reference dose or concentration, a cancer oral slope factor, cancer potency value, or an inhalation unit risk. CTV can be applied as an early prediction for toxicity information level for chemicals. Two parameters were selected to use in ToxPi software: cancer potency value and reference dose toxicity.

ToxPi software was applied to explain the toxicity of the selected chemicals. The selected parameters were created as slides in ToxPi, and each slide or chemical was given equal weight to calculate the value of toxicity. After converting all parameters into slides with equal weight, the software calculated the ToxPi score; the higher the score the higher the toxicity value for that chemical.

Table (5-1) lists the compounds that were selected to evaluate the sensor, their applications, mechanism of action (specific molecular targets to which the drug binds, such as an enzyme or receptor) and the calculated parameters from different sources to be used in the software. The average low concentration, in milligram of active ingredient per liter, represents the lowest concentration that can affect aquatic life. The active ingredient is the concentration of chemicals that is biologically active, as for example the active ingredient of the herbicide is the ingredient that kills weeds. The value of AC50 represents the concentration of a chemical that gives a half-maximal response. The reference dose (RfD) represents the maximum acceptable exposure of oral dose of a toxic substance. If human exposure level to a chemical substance is less than RfD, the risk is acceptable. 
Table 5.1 The EDCs and their relevant data that was used in ToxPi software

\begin{tabular}{|c|c|c|c|c|c|c|c|c|c|c|}
\hline $\begin{array}{c}\text { Raw } \\
\text { number }\end{array}$ & source & Cas No. & Name & Use & $\begin{array}{l}\text { Mechanism } \\
\text { of action }\end{array}$ & $\begin{array}{c}\text { Average lower } \\
\text { concentration } \\
\text { Al }(\mathrm{mg} / \mathrm{L})\end{array}$ & AC50 & $\begin{array}{l}\text { Assay of } \\
\text { AC50 }\end{array}$ & $\begin{array}{l}\text { Reference } \\
\text { Dose } \\
\text { toxicity } \\
\text { (mg/kg. } \\
\text { Day) }\end{array}$ & $\begin{array}{c}\text { Cancer } \\
\text { Potency } \\
\text { Value } \\
\text { risk } \\
\text { (mg/kg. } \\
\text { Day) } \\
\end{array}$ \\
\hline 1 & EPA & $50471-44-8$ & Vinclozolin & fungicide & $\begin{array}{l}\text { AR and ER } \\
\text { antagonists }\end{array}$ & 0.63 & 0.337 & NVS_NR_hAR & 0.025 & 0.571 \\
\hline 2 & EPA & $13311-84-7$ & Flutamide & Prodrug & $\begin{array}{r}\text { nonsteroidal } \\
\text { antiandrogen }\end{array}$ & 0.405 & 0.41 & NVS_NR_hAR & 0.0109 & 0.843 \\
\hline 3 & EPA & $97-23-4$ & Dichlorophen & fungicide & AR binding & 0.12 & 36.3 & $\begin{array}{c}\text { NVS_NR_hRAR_ } \\
\text { Antagonist }\end{array}$ & 0.0115 & 8.27 \\
\hline 4 & EPA & $80-05-7$ & BPA & $\begin{array}{l}\text { Surfactant } \\
\text { synthesis }\end{array}$ & AR binding & 0.00102 & 7.54 & NVS_NR_hAR & 0.05 & 8.02 \\
\hline 5 & EPA & $72-55-9$ & p,p' DDE & $\begin{array}{l}\text { surfactant } \\
\text { synthesis }\end{array}$ & $\begin{array}{l}\text { ER binding, } \\
\text { AR binding }\end{array}$ & 0.0003 & 21.9 & NVS_NR_cAR & $7.80 \mathrm{E}-05$ & 11.2 \\
\hline 6 & EPA & $50-29-3$ & p,p' DDT & pesticide & ER binding & 0.00007 & 0.07 & NVS_NR_bER & 0.000158 & 9.68 \\
\hline 7 & EPA & $2921-88-2$ & chlorpyrifos & insecticide & AR binding & 0.000027 & & & $3.00 \mathrm{E}-04$ & 0.618 \\
\hline 8 & EPA & $50-28-2$ & Estradiol & Medication & ER binding & 8.17162E-06 & $3.68 \mathrm{E}-03$ & NVS_NR_hAR & 0.00313 & 85.9 \\
\hline
\end{tabular}


5.2.2.7 The relation between the sensor response to testosterone and EDCs $\left(\lambda_{1} \& \lambda_{2}\right)$.

The optical response of the sensor to testosterone and 8 other chemicals was investigated. Testosterone MIP-sensors were incubated in solutions of variable concentration of each chemical and after equilibrium was reached, the reflectance spectra of the films was recorded. A shift in the peak wavelength was determined, comparing the before and after incubation spectra. The relationship between the concentration of each chemical and the shift of the peak wavelength reflected from the sensors caused by the incubation at such concentration was analyzed. The sensors are expected to show a correlation between concentration and wavelength shift for the testosterone, but also structurally similar compounds. Those compounds that have a better "fit" for the testosterone specific binding sites will be captured by the MIP to a larger extent and therefore produce a more significant peak wavelength shift.

The ratio of the peak wavelength shift of a chemical compound to the peak wavelength shift produced by the same concentration of testosterone is proposed as a measurement of the relative tendency of that chemical to occupy testosterone specific binding sites, being those natural or synthetic. The parameter $\lambda_{1}$ represents this ratio, as shown in equation 5-1.

$\lambda_{1, i}=\frac{\text { peak wavelength shift caused by substance } \mathrm{i}}{\text { peak wavelength shift caused by testosterone }}$

Our hypothesis was that the value of $\lambda_{1}$ correlates with the ED activity of the chemicals, a lower ratio meaning less ED activity expected. In order to test this hypothesis, a group of chemicals was tested by the testosterone MIP sensor. The results were classified according to the $\lambda_{1}$ value as: strong affinity binding chemicals (for high values of $\lambda_{1}$ ), 
moderate (intermediate values of $\lambda_{1}$ ), and weak/ inactive (low values of $\lambda_{1}$ ). The cutoff values for each group were used as fitting parameters for the experimental data.

An alternative method to quantify the relative response of the MIP sensor to a chemical vs. testosterone is expressed by the second parameter proposed in this work, $\lambda_{2}$, shown in equation 5-2. It represents the ratio of the concentration of testosterone solution that would produce the same peak wavelength shift of chemical to that of any chemical (i) when used with the MIP sensor.

$\lambda_{2, i}=\frac{\text { testosterone concentration that results at the same wavelength shift of substance } \mathrm{i}}{\text { concentration of substance i used for sensor incubation }}(5-2)$

As a weaker response of the MIP is expected for a random chemical than for the imprinted molecule (testosterone), a higher concentration of a chemical than that of testosterone will be needed in order to produce the same wavelength shift. With increasing affinity for the testosterone binding sites, lower aqueous concentrations will be required to produce the same effect on the MIP, with that of testosterone as the limiting lowest concentration. Therefore, the parameter $\lambda_{2}$ will take values $<1$, the higher $\lambda_{2}$, the higher affinity for testosterone binding sites for a chemical.

In this work, reference testosterone concentrations of $5 \mathrm{ppb}$ and $10 \mathrm{ppb}$ were chosen to compare the peak wavelength shifts of other chemicals tested. A low concentration was selected to ensure that a $\lambda_{2}$ existed; for a low affinity compound, a certain wavelength shift may not be attainable, as the concentrations needed to produce that effect in the sensor may be too high to be relevant or may be above its aqueous solubility.

\subsection{Results and discussion}

\subsubsection{Characterization of silica particles and MIP films}


The size of the commercial silica particles was investigated by SEM images, and the images analyzed under Esprit 2.1 software to confirm their size distribution. Several images of SEM with at least 300 of silica particles were investigated. The particle size was determined to be $300 \pm 8 \mathrm{~nm}$. Particles were deemed appropriate for their use in colloidal crystal fabrication if the standard deviation of the size distribution was below 5\%, which was confirmed for the silica particles used. This colloidal crystal was used as a template to prepare the 3D porous structure of the hydrogel film sensor (Figure 5.3a\&b).

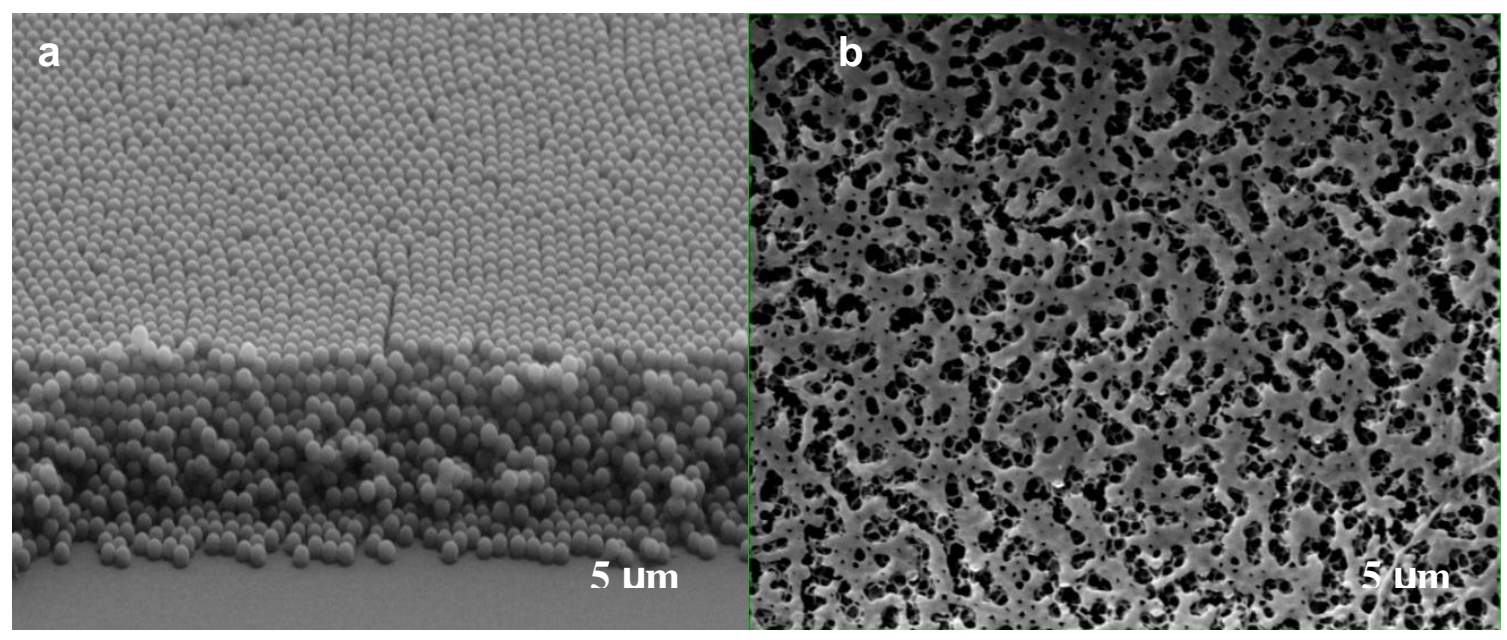

Figure 5.3. SEM images a) colloidal crystal of silica particles, b) nano-porous (top view) of MIP film

\subsubsection{The MIP response incubated in an aqueous solution of testosterone}

The sensor response was tested for different testosterone concentrations between $1 \mathrm{ppb}$ and $10 \mathrm{ppb}$, and the peak wavelength of the reflectance spectra shifted as expected. There was a correlation between the concentration of testosterone and the peak wavelength of the reflected light. At a higher concentration of testosterone, a higher wavelength shift was observed, as shown in Figure 5.4.

A calibration curve (response to incubation at variable testosterone concentration) was obtained for each sensor. This was needed due to limitations in the reproducibility of 
the MIP films. Although films were fabricated one by one in the laboratory with extreme care, some variability in the colloidal crystal morphology, incubation, and particle removal process proved to be inevitable. This variability is partly due to the fact that the sensor fabrication is a several step process that occurs over a several days period and is conducted manually by the researcher, one by one; additionally, the films are extremely sensitive, with minor dissimilarities being capable of producing significant differences in response.
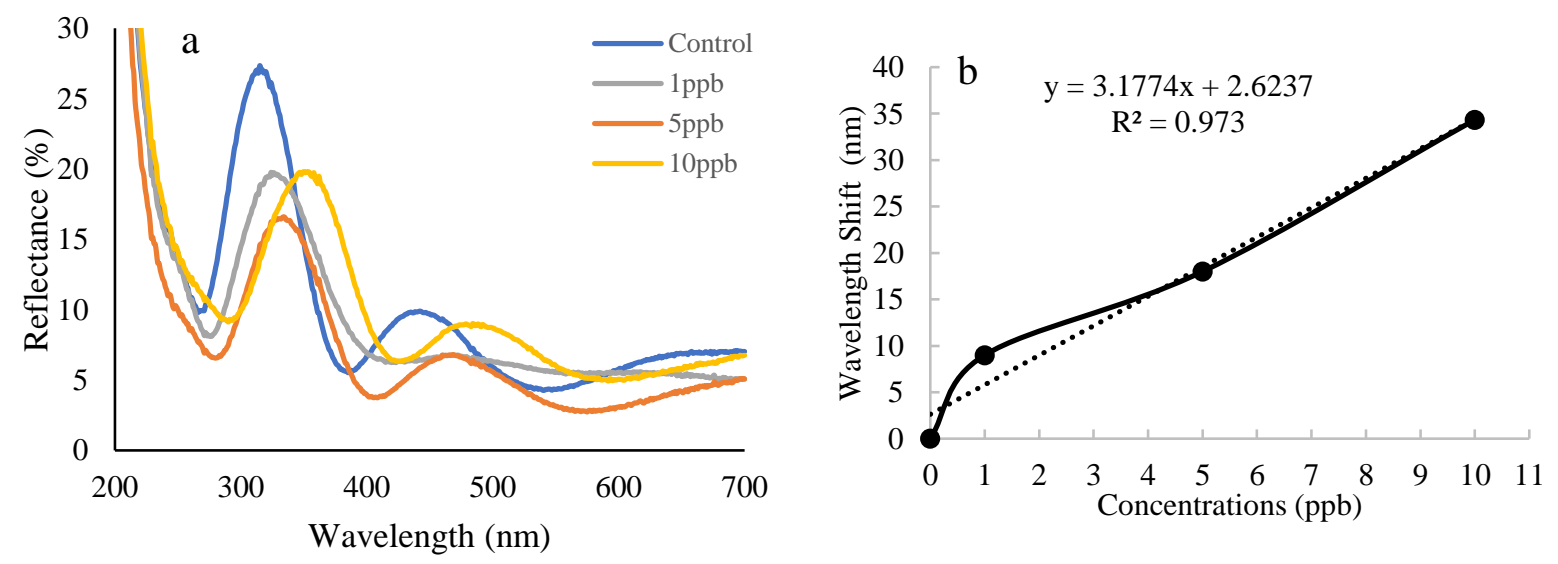

Figure 5.4. Optical properties of sensors: (a) reflectance spectra of MIP's at various concentrations of T. (b) diffraction peak shift for MIPs after incubation at various concentrations of T.

\subsubsection{Data Toxicity using ToxPi}

The results for ToxPi software resulted from the integration of three domains Ecotoxicity, in vitro bioassay method, and CTV predictor. The results are summarized in Figure (5.5). The blue color represents the AC50, red color represents average lower concentration, green color represents cancer potency value, and the brown color represents the reference dose value. 


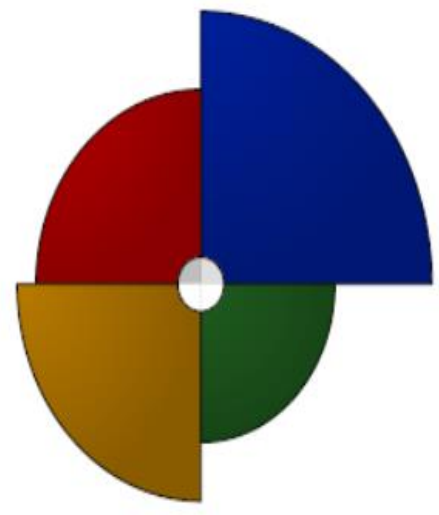

Dichlorophen

Score $=0.7408$

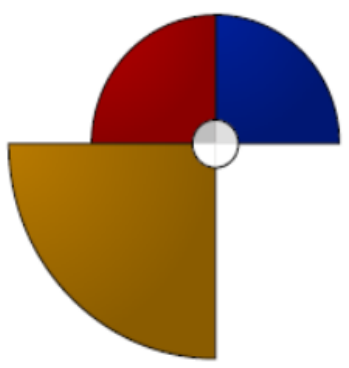

Vinclozolin

Score $=0.4680$

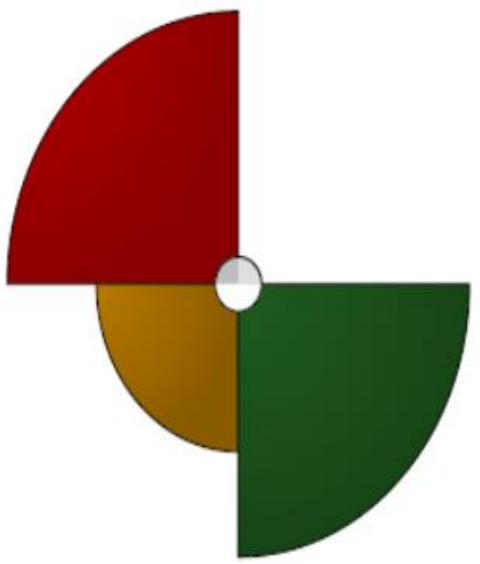

Estradiol

Score $=0.8428$

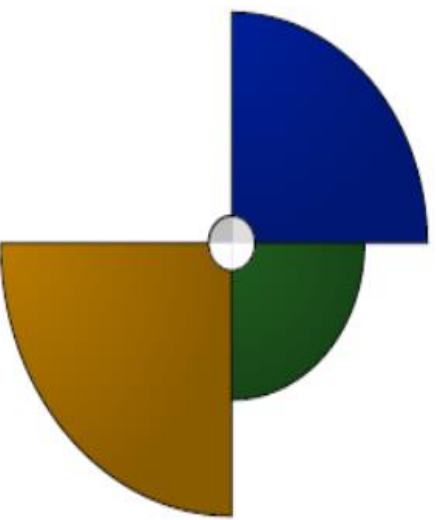

bisphenol A

Score $=0.5890$

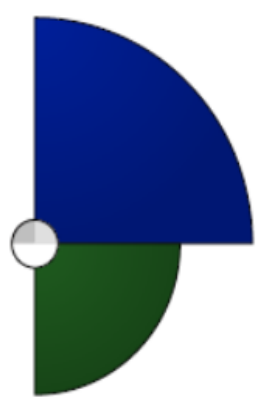

Flutamide Score $=0.3380$

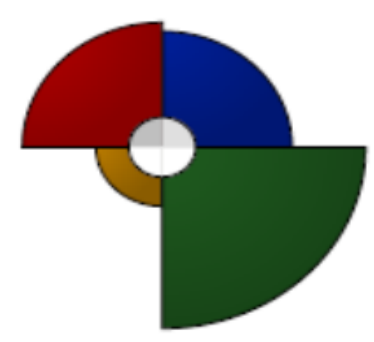

p,p' DDT

Score $=0.3360$ p.p' DDE

Score $=0.3847$
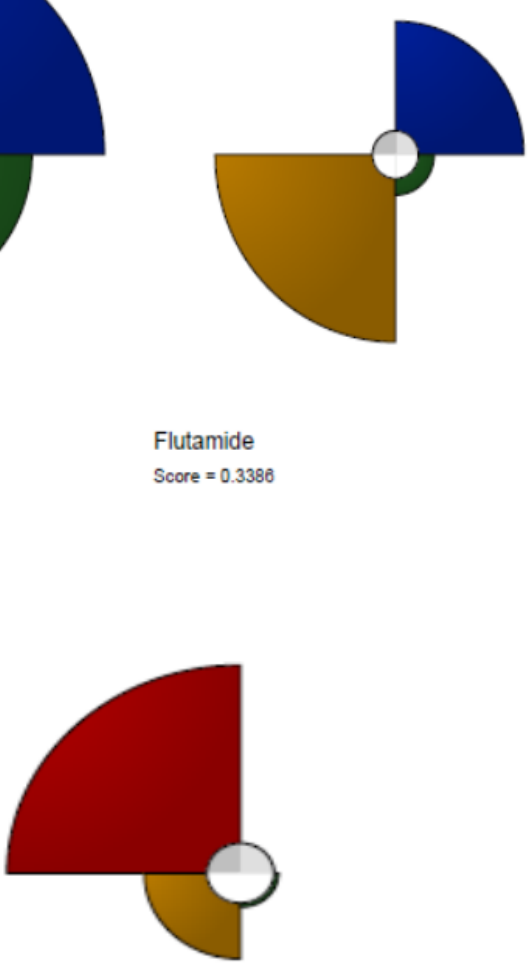

chlorpyrifos

Score $=0.2221$ 
Figure 5.5 Toxicological prioritization index score for the eight endocrine disrupting chemicals (simulated data) for a model combining source data into 4 slices (AC50 with blue color, average lower concentration with red color, reference dose toxicity with gold color, and cancer potency value with green color). For each slice, the distance that the arc extends from the origin is proportional to its relative evidence of concern (e.g. longer $=$ greater risk), and the radial angle (width) indicates its weight in the overall model.

The four slices represent the number of parameters that were selected. The distance from the origin (center) represents the potency, the slides with a larger radius has higher toxicity value. The width (in radians) of each slice represents the number of endpoints in each category, while the length of the slice indicates the overall degree of hazard. The ToxPi results showed that the Dichlorophene was the highest toxicity chemical with a value of 0.7489 , however, the chlorpyrifos was the lowest by a score of 0.2221 . The results showed that there was no effect for cancer potency value (green color) for VIN and CHL and very little effect for FLU. The AC50 ( blue color) did not appear to effect on EST and CHL. Average lower concentration (red color) did not appear to affect FLU, DDE, and $\mathrm{BPH}$. Whereas, the reference dose value affected on all the chemicals except DDE. DCP and DDT are the only two EDCs that affected by all the selected parameters. The ToxPi index of the chemicals was as follows (Dichlorophene, Estradiol, Bisphenol, Vinclozolin, DDE, Flutamide, DDT, and Chlorpyrifos) with the highest the value, the more the toxic chemical. The results showed that all the EDCs that used in this study are toxic in a different range, however, some of the parameters (slices) were missing when calculated in ToxPi which means that the parameter does not contribute to the toxicity of that chemical.

\subsubsection{The response of the MIPs to the EDCs}

The response of the sensor was investigated when incubated in testosterone and eight different endocrine disrupting chemicals: BPA, FLU, EST, DCP, DDT, DDE, CHL, 
and VIN. These EDCs were selected according to their effect on the endocrine system, toxicity, and the similarity of testosterone in chemical structure. These chemicals compete with the androgen or estrogen receptor leading to agonist or antagonist to the receptor. The activity of these chemicals to the receptors is different from chemical to others. Our goal is to use the testosterone sensor to predict their activity to that sensor and compare our results with the other methods that were used to study the affinity or activity of these chemicals to the receptor.

Figure 5.6 represents the comparison between the sensor response to testosterone and the response to EDCs at three different concentrations 1, 5, and $10 \mathrm{ppb}$. The results showed that the sensors had a higher affinity to testosterone than the other chemicals. It was hypothesized that because estradiol has a similar structure to testosterone it may occupy a fraction of the sites and prevent the binding of testosterone to the imprinted cavities. This was observed in our previous work [240] where estradiol showed to produce a response of the sensor. As expected, estradiol showed an increased response to the sensor with increasing concentration, starting with a $4 \mathrm{~nm}$ shift at $1 \mathrm{ppb}, 8 \mathrm{~nm}$ at $5 \mathrm{ppb}$, and 12 $\mathrm{nm}$ at $10 \mathrm{ppb}$. Most of the EDCs in $5 \mathrm{ppb}$ concentrations showed a wavelength shift of less than $50 \%$ of that produced by testosterone, at the same concentration except CHL and VIN. In general, all the EDCs showed a response of less than $34 \%$ at $10 \mathrm{ppb}$. The results explain that with the increasing concentrations the sensor exhibited a higher affinity to the testosterone than the EDCs.

\section{5}




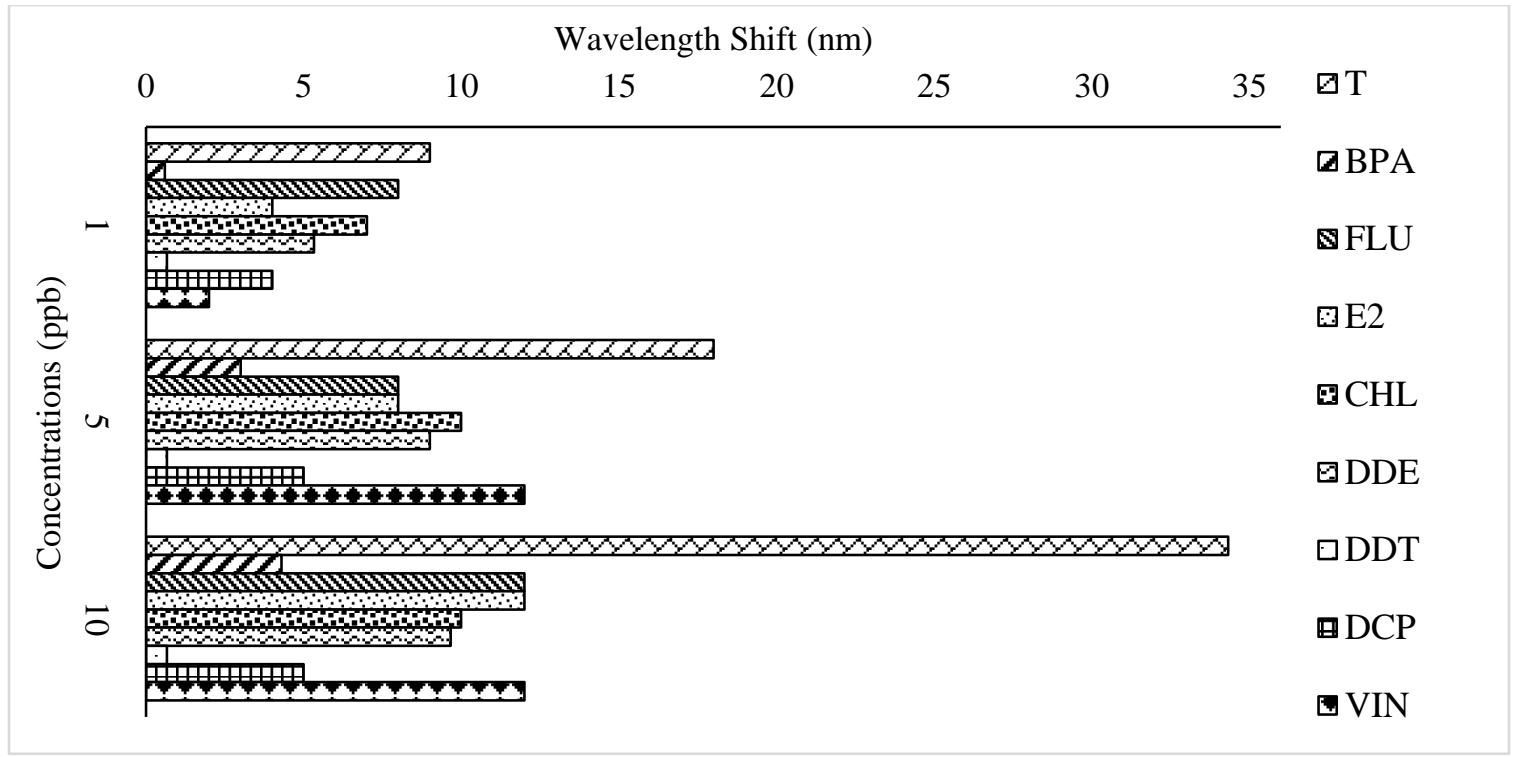

Figure 5.6 Peak wavelength shift for films incubated in solutions of variable concentration of testosterone and eight endocrine disrupting chemicals.

The parameters $\lambda_{1}$ and $\lambda_{2}$ were calculated as explained in the Methods and Materials section. Figure 5.7 shows the relationship between $\lambda_{1}$ and different concentrations 1, 5, 10 $\mathrm{ppb}$ of $\mathrm{T}$ and the investigated EDCs. It is clear that the binding affinity of the sensors to $\mathrm{T}$ was higher than the binding affinity to the EDCs because the binding site was specifically fabricated for the former molecule. At the lowest concentration tested, $1 \mathrm{ppb}$, there was a high response for DCP, CHL, and FLU. However, this is a very low concentration of $1 \mathrm{ppb}$ and part of that binding for these chemicals may be nonspecific binding resulting from adsorption on the surface. Besides, at lower concentrations, the shift of spectra for EDCs was low which led to making all shifting for all the chemicals within a specific range. On the other hand, the results of the other two concentrations 5 , and $10 \mathrm{ppb}$ showed that testosterone is dominant among the other chemicals. 


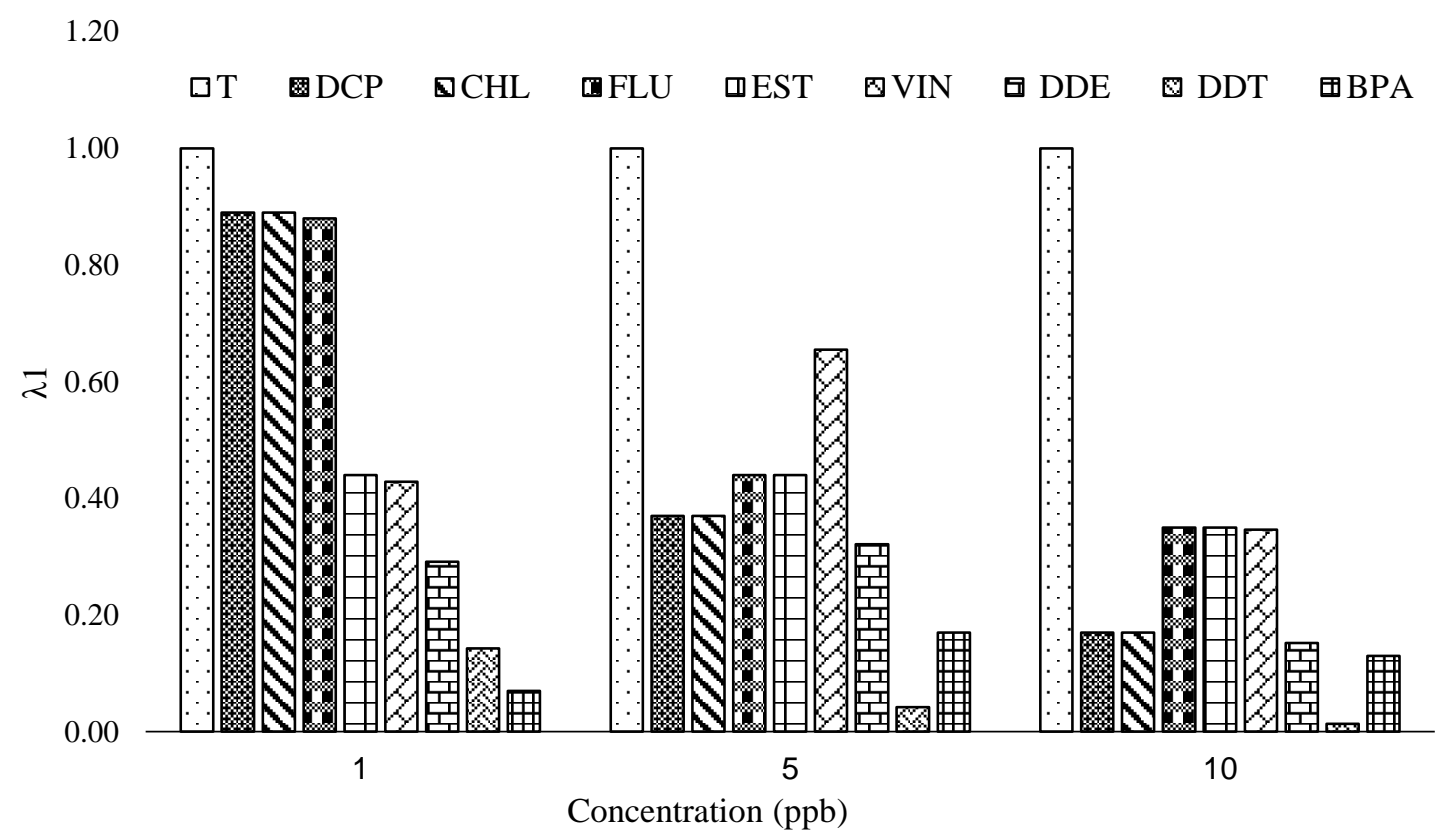

Figure 5.7 The relation between the response of EDCs to the response of $\mathrm{T}$ with concentrations of these chemicals.

Figure 5.8 showed the EDC's were classified according to equation (5-1) into four categories. A relation between the relative tendency of that chemical to occupy testosterone specific binding sites and those chemicals at 1,5 , and $10 \mathrm{ppb}$ was explained and the results showed that the EDCs were either moderate, weak or inactive as a compare with the testosterone for all the concentrations 1,5 and $10 \mathrm{ppb}$. Also, from the figure (5.8), the results showed that the BPA had a weak response for all concentrations. The lowest activity chemical was DDT, which was inactive for all concentrations and his toxicity was low (0.33). The high effective chemicals were the chemicals that had a high or moderate activity with high or moderate toxicity such as DCP, EST, and VIN. 

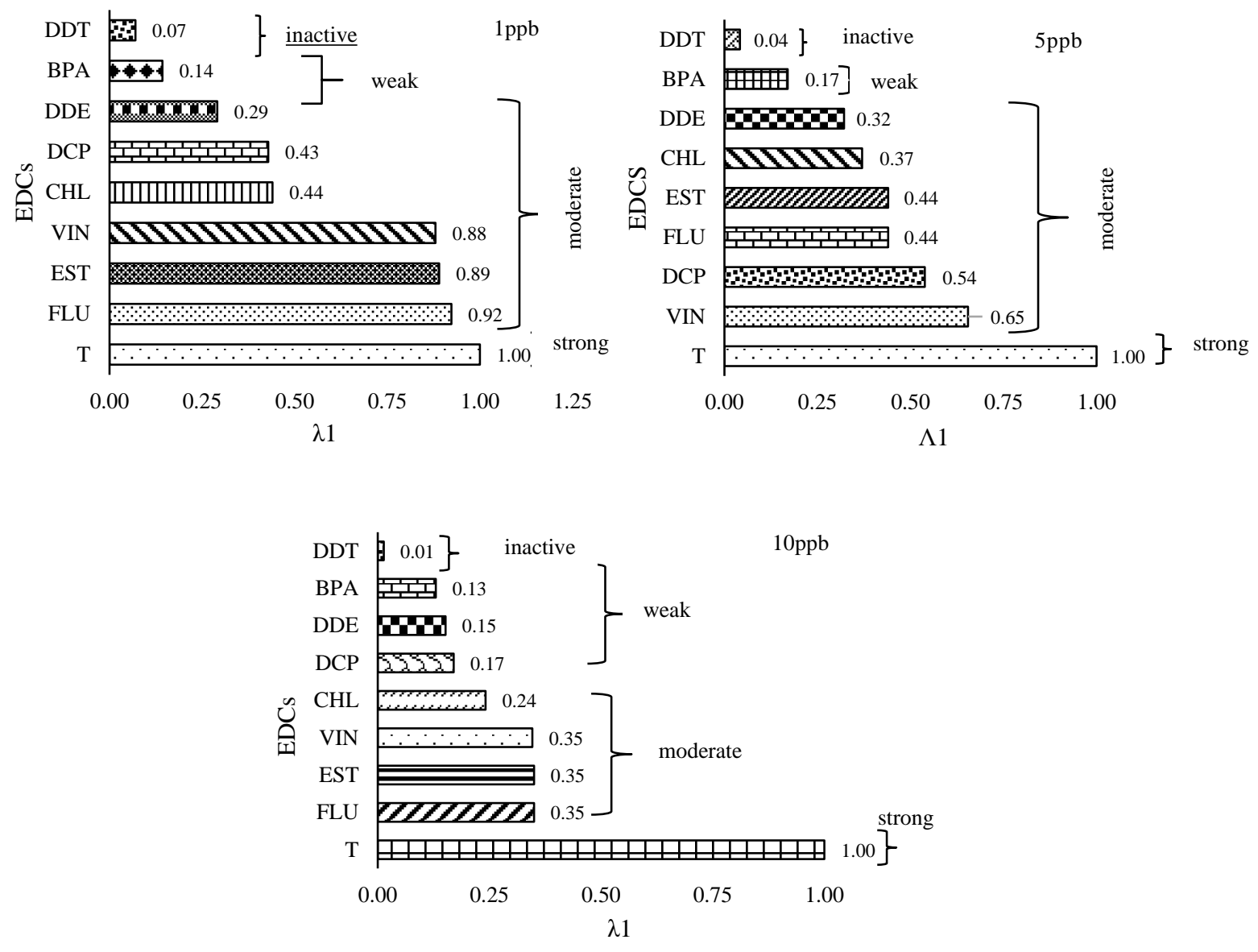

Figure 5.8 The binding affinity of testosterone as a comparison with the other EDCs at 1, 5, and $10 \mathrm{ppb}$.

Also, a comparison was implemented between our sensors and other methods that were used to study the affinity of chemicals to the androgen receptor. Fang and co-workers [280] used a competitive receptor binding assay to calculate the relative binding affinity (RBA) of 202 chemicals. The relative binding affinity was calculated by dividing the IC50 of R1881 by the IC50 of the competitor (test chemical) and was expressed as a percent. Then, The RBA was classified into four activity groups concerning their RBA range, strong, moderate, weak, and inactive groups. Table 5.2 represents the results that were gotten from our sensors and the results of Fang and co-workers. The comparison was implemented for just seven chemicals T, FLU, EST, VIN, DDE, BPH, and DDT. By comparing our results for concentrations 1,5 , and $10 \mathrm{ppb}$ to the Fang results, the tabled 
showed that four of our chemicals (T, EST, DDE, and BPH) had the same classification of Fang results at concentrations 1 and $5 \mathrm{ppb}$, and three chemicals at $10 \mathrm{ppb}$ T, EST, and BPH. Testosterone had a strong binding affinity to our sensor in all concentrations as a result of his binding site found inside the sensor, and it was strong also in Fang results.

Table 5.2 Comparison of EDCs sensor affinity with RBA of Fang method

\begin{tabular}{|c|c|c|c|c|}
\hline ED CS & $\begin{array}{c}\text { Classification @ } \\
1 p p b\end{array}$ & $\begin{array}{c}\text { Classification @ } \\
5 \mathrm{ppb}\end{array}$ & $\begin{array}{c}\text { Classification @ } 10 \\
\text { ppb }\end{array}$ & Fang classification \\
\hline $\mathrm{T}$ & strong & strong & strong & strong \\
\hline FLU & moderate & Moderate & moderate & weak \\
\hline EST & moderate & Moderate & moderate & moderate \\
\hline VIN & moderate & Moderate & moderate & weak \\
\hline $\mathrm{CHL}$ & moderate & moderate & Moderate & ND \\
\hline $\mathrm{DCP}$ & moderate & moderate & moderate & ND \\
\hline DDE & moderate & Moderate & weak & moderate \\
\hline BPA & Weak & Weak & weak & weak \\
\hline DDT & inactive & inactive & inactive & moderate \\
\hline
\end{tabular}

One of the EDCs was inactive which was DDT whereas it was moderate according to Fang study. The mechanism of action of DDT is through binding to the estrogen receptors, which explains the reason for being inactive since the MIP sensor mimics androgenic receptors. BPA exhibited a weak response with calculated $\lambda_{1}$ values of all the concentrations 1,5 , and $10 \mathrm{ppb}$ because its shift compared to testosterone shift was very low, and also the same classification for BPA in Fang's method. The CHL and DCP were not included in the Fang study, however, in our study, they were classified as a moderate affinity at all concentrations. CHL started with $\lambda 1$ equal to 0.44 at $1 \mathrm{ppb}$ and reduced to 0.24 at $10 \mathrm{ppb}$, whereas the DCP had a value of 0.43 at $1 \mathrm{ppb}$ and reduced to 0.17 at $10 \mathrm{ppb}$, both of those ranges were considered as a moderate affinity to sensor in our classification. Both FLU and VIN were considered a moderate affinity according to our classification, 
but they were considered a weak affinity in Fang classification. In general, 57\% of our classification was identical with Fang classification which means that our sensor can be used as a pre method to study the affinity of EDCs binding to AR after trying to test it with more EDCs to give a clear image about his ability.

The $\lambda_{2}$ was calculated by dividing the concentration of testosterone resulting from the EDCs wavelength shift to the actual concentration of chemicals produced that shift. The higher the value of $\lambda_{2}$ the higher activity of the EDCs. Our hypothesis was that the values of $\lambda_{2}$ are less than 1 for all EDCs because of the specificity of the sensor to the target (testosterone) which makes it harder for the other chemicals to bind.

Figure 5.9 represents the percent of the value of EDCs that occupied the testosterone site inside the sensor for both concentrations 5, $10 \mathrm{ppb}$. The results showed small cavities were occupied by concentrations of EDCs as a result of differences in structures and specificity of the sensor to the testosterone as a target.

The $\lambda_{2}$ for DDT was between $1-2 \%$ for both $5 \mathrm{ppb}$ and $10 \mathrm{ppb}$ which means that the wavelength shift produced by DDT is equivalent to a very low testosterone concentration, where $5 \mathrm{ppb}$ concentration of DDT equavilent to $0.1 \mathrm{ppb}$ of testosterone. In other words, DDT concentration was filling just $2 \%$ of the testosterone cavities and we need to increase the concentration 50 times to get the same wavelength shift of testosterone at $5 \mathrm{ppb}$.

It is clear from Figure 5.9 that the specificity of the sensor is clearer to the testosterone with the increasing of EDCs concentrations, where all the values of $\lambda_{2}$ for all chemicals reduced with increasing the concentration from $5 \mathrm{ppb}$ to $10 \mathrm{ppb}$. This was because with increasing the EDCs concentrations, it occupied the same binding site. The 
wavelength shift resulting from occupying produced the same testosterone concentration.

The activity of the chemicals was determined to be between 1-5\% for DDT and BPH, between 5-15\% for DDE, between 18-34\% for EST, FLU, CHL, and DCP, except VIN who start with $66 \%$ and then reduce to $33 \%$ and $100 \%$ for T.

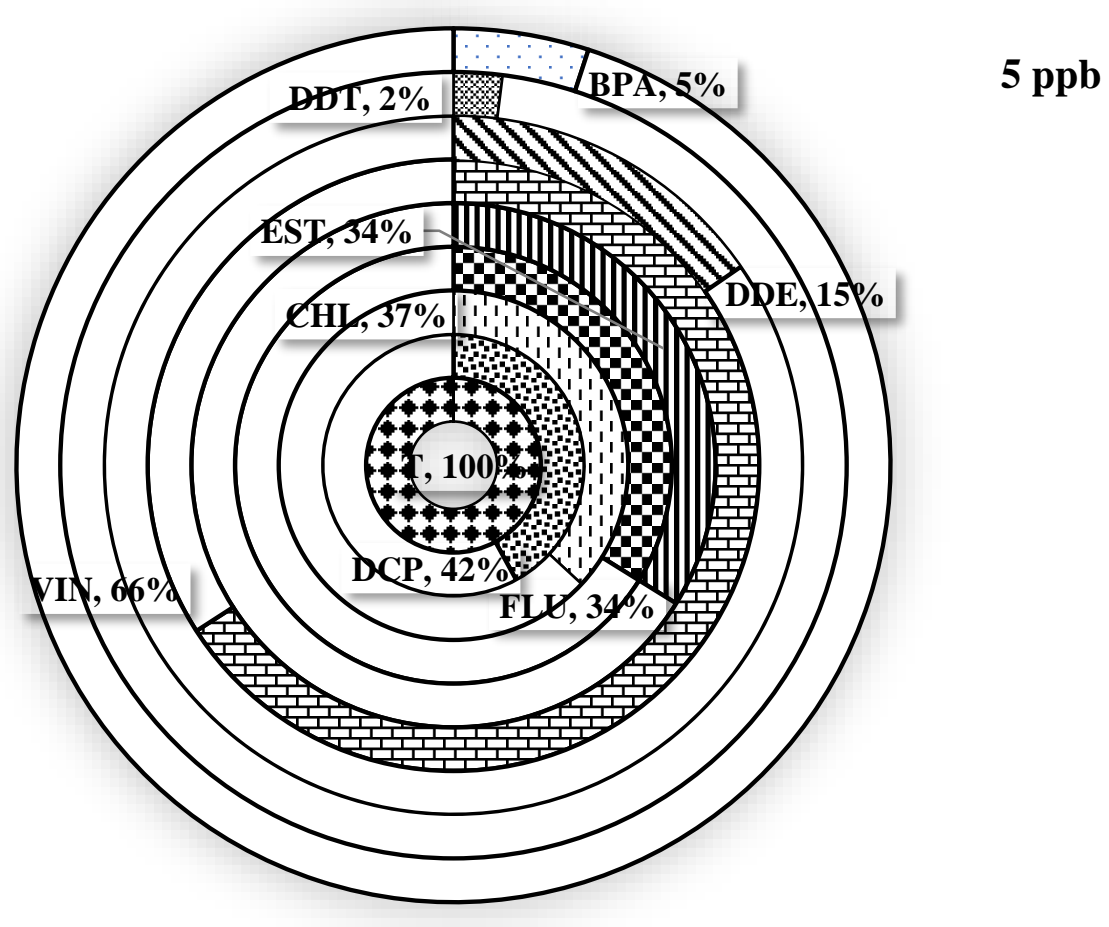




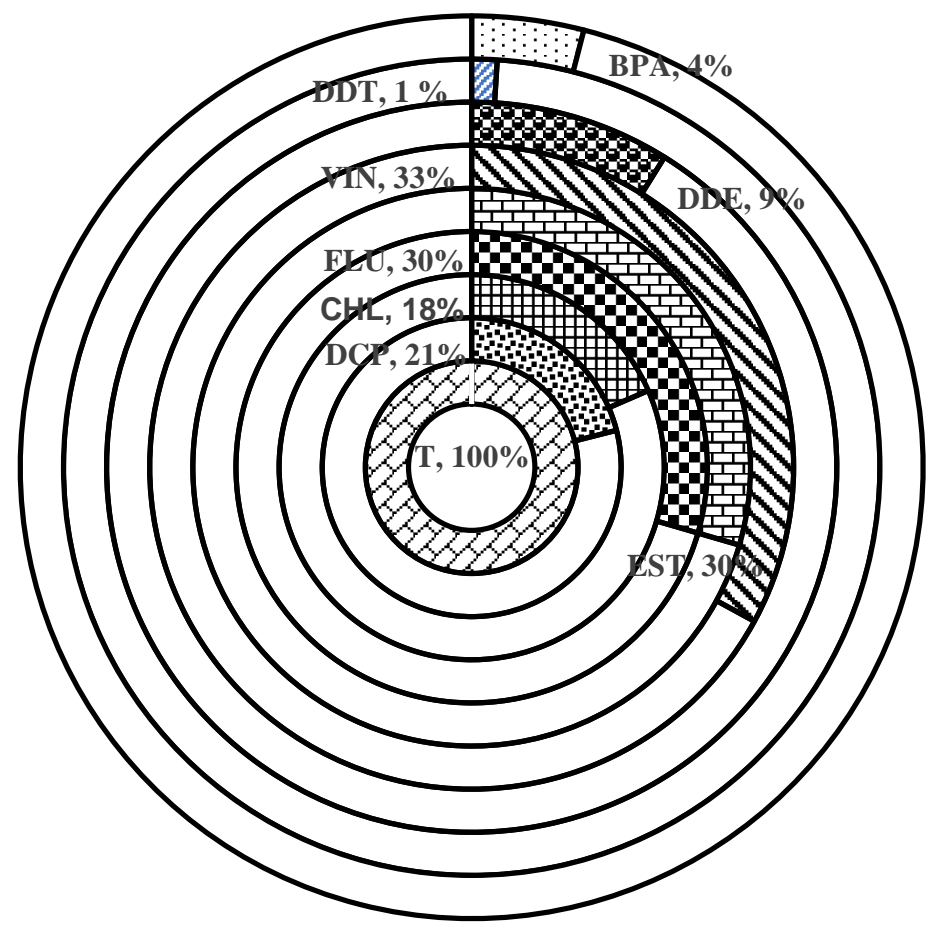

10ppb

Figure 5.9 The percent value for the EDCs concentrations as compared with testosterone concentration at the same wavelength shift at concentrations $5 \mathrm{ppb}$ and $10 \mathrm{ppb}$.

\subsection{Conclusion}

In this study, eight EDCs were studied to investigate their affinity for the binding sites of the T MIP sensor. The wavelength shift produced in an optical sensor by each chemical in a comparison with $\mathrm{T}$ was studied for three different concentrations 1,5 , and $10 \mathrm{ppb}$. The chemicals were selected according to their similarity to T structure, toxicity, structures, and androgen or estrogen activity. The study explained that there is a large difference in the wavelength shift produced by EDCs and that produced by the $\mathrm{T}$ at concentrations $5 \mathrm{ppb}$ and $10 \mathrm{ppb}$. However, some chemicals showed response at 1ppb. Based on the results of the study, the chemicals were classified into 4 categories, according to their response: strong affinity (T), moderate (CHL, VIN, EST, and FLU), weak (BPA, DDE, and DCP), and inactive (DDT). Also, the percent activity showed that the selected chemicals had 
lower adsorption to the binding site of the sensor in comparison with testosterone. In general, the sensor constitutes a promising idea to screen for the ED affinity to the androgen receptor. However, more EDCs need to be tested to expand and confirm the findings of this work. 


\section{CHAPTER 6}

\section{CONCLUSIONS AND FUTURE DIRECTIONS}

In this study, an optical sensor was fabricated to detect the testosterone in water. The optical film was tested first by the HPLC instrument to detect the testosterone. After that, the sensors were used on the surface of PMMA to detect the testosterone by using UV-Visible spectroscopy. The results showed that the sensors were highly specific detection to the target as a comparison with the other hormones. Also, the detection limit for the sensor by using HPLC was 10ppb with an equilibrium time equal to $30 \mathrm{~min}$. the sensors were able to be reused at least five times with the same efficiency.

These types of sensors were applied to detect the free testosterone in blood samples. The ratio of polymerization solution was optimized, and the equilibrium time was reached to $2 \mathrm{~min}$. The sensors were tested in blood samples. Two types of blood were used without pretreatment: horse and human blood. The sensor showed a clear wavelength shifting with horse blood. However, it did not detect the target with very low concentration in human blood samples.

Finally, the $\mathrm{T}$ sensor was investigated as a first screening to detect potential endocrine disruptive chemicals. Based on the sensor results, chemicals were classified into four categories according to their response to the testosterone sensor which is strong affinity (T), moderate (CHL, VIN, EST, and FLU), weak (BPH, DDE, and DCP), and inactive (DDT). In general, $57 \%$ of our classification was identical with other methods that were used to study the affinity of chemicals to the androgen receptor. This research 
represents a preliminary study to investigate the affinity for overall EDCs in a simpler and faster way than traditional methods.

More research is needed for the detection of testosterone in aqueous solution such as providing an expanded study for the detection of testosterone in blood samples. Also, applied a comprehensive study for the natural, synthetic, and environmental chemicals for binding by testosterone sensor. 


\section{References}

[1] C. He, Y. Long, J. Pan, K. Li, and F. Liu, "A method for coating colloidal particles with molecularly imprinted silica films.," J. Mater. Chem., vol. 18, no. 24, pp. 2849$2854,2008$.

[2] S. Wagner, J. Bell, M. Biyikal, K. Gawlitza, and K. Rurack, "Integrating fluorescent molecularly imprinted polymer (MIP) sensor particles with a modular microfluidic platform for nanomolar small-molecule detection directly in aqueous samples," Biosens. Bioelectron., vol. 99, no. July 2017, pp. 244-250, 2018.

[3] K. Haupt, Molecular Imprinting, 1 st Editi. Compiegne-France, 2012.

[4] M. J. Abdin, Z. Altintas, and I. E. Tothill, "In silico designed nanoMIP based optical sensor for endotoxins monitoring," Biosens. Bioelectron., vol. 67, pp. 177-183, 2015.

[5] C. D. Kassotis, D. E. Tillitt, J. W. Davis, A. M. Hormann, and S. C. Nagel, "Estrogen and androgen receptor activities of hydraulic fracturing chemicals and surface and ground water in a drilling-dense region," Endocrinology, vol. 155, no. 3, pp. 897907, 2014.

[6] T. F. T. Omar, A. Z. Aris, F. M. Yusoff, and S. Mustafa, "An improved SPE-LCMS/MS method for multiclass endocrine disrupting compound determination in tropical estuarine sediments," Talanta, vol. 173, no. May, pp. 51-59, 2017.

[7] S. Y. Wee and A. Z. Aris, "Endocrine disrupting compounds in drinking water supply system and human health risk implication," Environ. Int., vol. 106, no. May, pp. 207-233, 2017. 
[8] N. A. H. Ismail, S. Y. Wee, and A. Z. Aris, "Multi-class of endocrine disrupting compounds in aquaculture ecosystems and health impacts in exposed biota," Chemosphere, vol. 188, pp. 375-388, 2017.

[9] J.W. Birkett and J. N. Laster, endocrine Disrupters in Wastewater and Sludge Treatment Processes. USA: Lewis Publishers, 2003.

[10] A. Z. Aris, A. S. Shamsuddin, and S. M. Praveena, "Occurrence of 17aethynylestradiol (EE2) in the environment and effect on exposed biota: A review," Environment International, vol. 69. pp. 104-119, 2014.

[11] M. Zhao and G. Shen, "The application of molecularly imprinted polymers," $J$. Mater. Sci. Chem. Eng., vol. 3, pp. 87-89, 2015.

[12] J. Wackerlig and P. A. Lieberzeit, "Molecularly imprinted polymer nanoparticles in chemical sensing - Synthesis, characterisation and application," Sensors Actuators B Chem., vol. 207, pp. 144-157, 2015.

[13] A. Pardo, L. Mespouille, P. Dubois, B. Blankert, and P. Duez, "Molecularly imprinted polymers: compromise between flexibility and rigidity for improving capture of template analogues," Chemistry, vol. 20, no. 12, pp. 3500-3509, 2014.

[14] S. Asman, S. Mohamad, and N. M. Sarih, "Exploiting $\beta$-Cyclodextrin in molecular imprinting for achieving recognition of benzylparaben in aqueous media," Int. J. Mol. Sci., vol. 16, no. 2, pp. 3656-3676, 2015.

[15] S. Pardeshi, R. Dhodapkar, and A. Kumar, "Molecularly imprinted microspheres and nanoparticles prepared using precipitation polymerisation method for selective 
extraction of gallic acid from Emblica officinalis," Food Chem., vol. 146, pp. 385393, 2014.

[16] W. L. Yang, S. M. Huang, Q. Z. Wu, and J. F. He, "Properties evaluation and separation application of naringin-imprinted polymers prepared by a covalent imprinting method based on boronate ester," J. Polym. Res., vol. 21, no. 4, p. 383, 2014.

[17] T. Kamra, S. Chaudhary, C. Xu, L. Montelius, J. Schnadt, and L. Ye, "Covalent immobilization of molecularly imprinted polymer nanoparticles on a gold surface using carbodiimide coupling for chemical sensing," J. Colloid Interface Sci., vol. 461, pp. 1-8, 2016.

[18] Y. Tang et al., "Ultrasensitive detection of clenbuterol by a covalent imprinted polymer as a biomimetic antibody," Food Chem., vol. 228, pp. 62-69, 2017.

[19] L. Zhou, Y. Kong, S. Zhong, and X. Zhou, "Preparation and characterization of molecularly imprinted organic-inorganic hybrid materials by sol-gel processing for selective recognition of ibuprofen," J. Sol-Gel Sci. Technol., vol. 66, no. 1, pp. 5967, 2013.

[20] J. Zhan, G. Fang, Z. Yan, M. Pan, C. Liu, and S. Wang, "Preparation of a semicovalent, molecularly surface imprinted polymer for the rapid determination of trace acid orange II in food and environmental samples," Anal. Bioanal. Chem., vol. 405, no. 19, pp. 6353-6363, 2013.

[21] M. Dabrowski et al., "Hierarchical templating in deposition of semi-covalently imprinted inverse opal polythiophene film for femtomolar determination of human 
serum albumin,” Biosens. Bioelectron., vol. 94, no. December 2016, pp. 155-161, 2017.

[22] K. K. Tadi and R. V. Motghare, "Rational synthesis of pindolol imprinted polymer by non-covalent protocol based on computational approach," J. Mol. Model., vol. 19, no. 8, pp. 3385-3396, 2013.

[23] N. Henry, P. Favetta, R. Delépée, J. M. Seigneuret, and L. A. Agrofoglio, "Synthesis of a molecularly imprinted polymer to isolate glucosamine from plant extracts by an ionic-non-covalent dual approach," Int. J. Cosmet. Sci., vol. 37, no. 2, pp. 196-206, 2015.

[24] Y. Han et al., "Computer-aided design of molecularly imprinted polymers for recognition of atrazine," Comput. Theor. Chem., vol. 1121, pp. 29-34, 2017.

[25] X.-H. Wang, J. Zhang, C. Peng, Q. Dong, Y.-P. Huang, and Z.-S. Liu, "Comparison of multi-recognition molecularly imprinted polymers for recognition of melamine, cyromazine, triamterene, and trimethoprim," Anal. Bioanal. Chem., vol. 407, no. 23, pp. 7145-7155, 2015.

[26] J. Guo, Y. Wang, Y. Liu, C. Zhang, and Y. Zhou, "Magnetic-graphene based molecularly imprinted polymer nanocomposite for the recognition of bovine hemoglobin,” Talanta, vol. 144, pp. 411-419, 2015.

[27] Y. Suwanwong, S. Kulkeratiyut, V. Prachayasittikul, and S. Boonpangrak, "Effects of Polymerization Methods and Functional Monomers on Curcumin Imprinted Polymer Properties," Sep. Sci. Technol., vol. 49, no. 7, pp. 1086-1095, 2014. 
[28] S. C. and F. N.-V. Maria C. Moreno Bondi, Javier L. Urraca, Handbook of Molecularly Imprinted Polymers "Ch 2: Preparation of Molecularly Imprinted Polymers,” no. January. Smithers Rapra, 2013.

[29] R. C. Tenzin Pasang, "Synthesis and Characterization of Methacrylic Acid-Based Molecular Imprinted Polymers by Positron Lifetime Spectroscopy Tenzin,” Polym. Eng. Sci., vol. 54, no. 3, pp. 667-674, 2014.

[30] S. O. Dima et al., "Thermal analyses as tools for proving the molecular imprinting with diosgenin and sclareol in acrylic copolymer matrices," J. Therm. Anal. Calorim., vol. 120, pp. 1107-1118, 2015.

[31] R. Ma and Y. Shi, "Magnetic molecularly imprinted polymer for the selective extraction of quercetagetin from Calendula of fi cinalis extract," Talanta, vol. 134, pp. 650-656, 2015.

[32] P. Ma et al., "Surface imprinted polymers for oil denitrification with the combination of computational simulation and multi-template molecular imprinting," Polym. Adv. Technol., vol. 26, no. 5, pp. 476-486, 2015.

[33] C. meng Dai, J. Zhang, Y. lei Zhang, X. fei Zhou, Y. ping Duan, and S. guang Liu, "Removal of carbamazepine and clofibric acid from water using double templatesmolecularly imprinted polymers," Environ. Sci. Pollut. Res., vol. 20, no. 8, pp. 5492-5501, 2013.

[34] D. Mutavdzic Pavlovic, K. Niksic, S. Livazovic, I. Brnardic, and A. Anzlovar, "Preparation and application of sulfaguanidine-imprinted polymer on solid-phase extraction of pharmaceuticals from water," Talanta, vol. 131, pp. 99-107, 2015. 
[35] S. E. Diltemiz, D. Hür, R. Keçili, A. Ersöz, and R. Say, "New synthesis method for 4-MAPBA monomer and using for the recognition of IgM and mannose with MIPbased QCM sensors.," Analyst, vol. 138, no. 5, pp. 1558-63, 2013.

[36] A. Mehdinia, S. Dadkhah, T. Baradaran, and A. Jabbari, "Design of a surfaceimmobilized 4-nitrophenol molecularly imprinted polymer via pre-grafting amino functional materials on magnetic nanoparticles," J. Chromatogr. A, vol. 1364, pp. $12-19,2014$.

[37] S. Li, D. Du, J. Huang, H. Tu, Y. Yang, and A. Zhang, “One-step electrodeposition of a molecularly imprinting chitosan/phenyltrimethoxysilane/AuNPs hybrid film and its application in the selective determination of p-nitrophenol.," Analyst, vol. 138, no. 9, pp. 2761-8, May 2013.

[38] J. C. Yang, H. K. Shin, S. W. Hong, and J. Y. Park, "Lithographically patterned molecularly imprinted polymer for gravimetric detection of trace atrazine," Sensors Actuators, B Chem., vol. 216, pp. 476-481, 2015.

[39] N. Cennamo, G. D’Agostino, R. Galatus, L. Bibbò, M. Pesavento, and L. Zeni, "Sensors based on surface plasmon resonance in a plastic optical fiber for the detection of trinitrotoluene," Sensors Actuators, B Chem., vol. 188, pp. 221-226, 2013.

[40] T. S. Anirudhan and S. Alexander, "Multiwalled carbon nanotube based molecular imprinted polymer for trace determination of 2,4-dichlorophenoxyaceticacid in natural water samples using a potentiometric method," Appl. Surf. Sci., vol. 303, pp. 180-186, 2014. 
[41] Y. Li, T. Chen, B. Gao, and Z. Qiao, "Preparation of atrazine surface-imprinted material MIP-PSSS/SiO2 and study on its molecule recognition character," Korean J. Chem. Eng., vol. 31, no. 5, pp. 896-904, 2014.

[42] S. N. Egli, "Thin-Film Molecularly Imprinted Polymers for Detection Systems for Polycyclic Aromatic,” Memorial University of Newfoundland, 2014.

[43] H. Hashemi-Moghaddam and F. Abbasi, "Synthesis of Molecularly Imprinted Polymers Coated on Silica Nanoparticles for Removal of P-Nitrophenol from Crude Pharmaceuticals," Pharm. Chem. J., vol. 49, no. 4, pp. 280-286, 2015.

[44] J. J. Torres, N. Gsponer, C. L. Ram??rez, D. M. A. Vera, H. A. Montejano, and C. A. Chesta, "Experimental and theoretical studies on the enantioselectivity of molecularly imprinted polymers prepared with a chiral functional monomer," $J$. Chromatogr. A, vol. 1266, pp. 24-33, 2012.

[45] E. Lee, D.-W. Park, J.-O. Lee, D. S. Kim, B. H. Lee, and B. S. Kim, "Molecularly imprinted polymers immobilized on carbon nanotube," Colloids Surfaces A Physicochem. Eng. Asp., vol. 313-314, pp. 202-206, 2008.

[46] Y. Cai, Y. Chen, X. Hong, Z. Liu, and W. Yuan, "Porous microsphere and its applications," Int. J. Nanomedicine, vol. 8, pp. 1111-1120, 2013.

[47] B. Brooks, "Suspension polymerization processes," Chem. Eng. Technol., vol. 33, no. 11, pp. 1737-1744, 2010.

[48] L.-W. Qian et al., "Thermal preparation of lysozyme-imprinted microspheres by using ionic liquid as a stabilizer," Anal. Bioanal. Chem., vol. 406, no. 28, pp. 7221- 
7231, 2014.

[49] A. M. Florea, A. Sarbu, D. Donescu, A.-L. Radu, A. Zaharia, and T.-V. Iordache, "The structure effect upon gallic acid re-binding in molecularly imprinted organosilica," J. Sol-Gel Sci. Technol., vol. 76, no. 3, pp. 529-541, 2015.

[50] Y. Zhang, J. Ding, and S. Gong, "Preparation of molecularly imprinted polymers for vanillin via reversible addition-fragmentation chain transfer suspension polymerization,” J. Appl. Polym. Sci., vol. 128, pp. 2927-2932, 2013.

[51] Y. Suwanwong, S. Kulkeratiyut, V. Prachayasittikul, and S. Boonpangrak, "Effects of Polymerization Methods and Functional Monomers on Curcumin Imprinted Polymer Properties,” Sep. Sci. Technol., vol. 49, no. 7, pp. 1086-1095, 2014.

[52] H. Sun, J.-P. Lai, F. Chen, and D.-R. Zhu, "Molecularly imprinted microspheres synthesized by a simple, fast, and universal suspension polymerization for selective extraction of the topical anesthetic benzocaine in human serum and fish tissues," Anal. Bioanal. Chem., vol. 407, pp. 1745-1752, 2015.

[53] L. Ye, P. a. G. Cormack, and K. Mosbach, "Molecularly imprinted monodisperse microspheres for competitive radioassay," Anal. Commun., vol. 36, no. 2, pp. 3538, 1999.

[54] G. L. Li, H. Möhwald, and D. G. Shchukin, "Precipitation polymerization for fabrication of complex core-shell hybrid particles and hollow structures.," Chem. Soc. Rev., vol. 42, no. 8, pp. 3628-46, 2013.

[55] M. Liu, Y. Li, J. Han, and X. Dong, "Synthesis of tetracycline-imprinted polymer 
microspheres by reversible addition-fragmentation chain-transfer precipitation polymerization using polyethylene glycol as a coporogen," J. Sep. Sci., vol. 37, no. 9-10, pp. 1118-1125, 2014.

[56] F.-X. Gao, X.-T. Ma, X.-W. He, W.-Y. Li, and Y.-K. Zhang, "Smart surface imprinting polymer nanospheres for selective recognition and separation of glycoprotein," Colloids Surfaces A Physicochem. Eng. Asp., vol. 433, pp. 191-199, 2013.

[57] S. Azodi-Deilami, M. Abdouss, D. Kordestani, and Z. Shariatinia, "Preparation of $\mathrm{N}, \mathrm{N}$-p-phenylene bismethacryl amide as a novel cross-link agent for synthesis and characterization of the core-shell magnetic molecularly imprinted polymer nanoparticles," J. Mater. Sci. Mater. Med., vol. 25, pp. 645-656, 2014.

[58] T. Yao et al., "Enhancement of surface plasmon resonance signals using a MIP/GNPs/rGO nano-hybrid film for the rapid detection of ractopamine," Biosens. Bioelectron., vol. 75, pp. 96-100, 2016.

[59] F. Tan et al., "Molecularly imprinted polymer/mesoporous carbon nanoparticles as electrode sensing material for selective detection of ofloxacin," Mater. Lett., vol. 129, pp. 95-97, 2014.

[60] S. Cao, J. Chen, W. Sheng, W. Wu, Z. Zhao, and F. Long, "The fabrication and development of molecularly imprinted polymer-based sensors for environmental application.," in Mol. Imprinted Sens. overview and applications, Elsevier Science \& Technology, 2012, pp. 57-72.

[61] Q. Liu, Y. Feng, S. Huang, Q. Wu, and J. He, "Preparation of ordered macroporous 
cinchonine molecularly imprinted polymers and comparative study of their structure and binding properties with traditional bulk molecularly imprinted polymers," Polym. Int., vol. 64, no. 11, pp. 1594-1599, 2015.

[62] A. Mehdinia, T. Baradaran, A. Jabbari, M. O. Aziz-zanjani, and E. Ziaei, "Magnetic molecularly imprinted nanoparticles based on grafting polymerization for selective detection of 4-nitrophenol in aqueous samples," J. Chromatogr. A, vol. 1283, pp. 82-88, 2013.

[63] Y. Hiratsuka, N. Funaya, H. Matsunaga, and J. Haginaka, "Preparation of magnetic molecularly imprinted polymers for bisphenol $\mathrm{A}$ and its analogues and their application to the assay of bisphenol A in river water," J. Pharm. Biomed. Anal., vol. 75, pp. 180-185, 2013.

[64] M. Yang, Y. Guo, Q. Wu, Y. Luan, and G. Wang, "Synthesis and properties of amphiphilic nonspherical SPS/PS composite particles by multi-step seeded swelling polymerization," Polym. (United Kingdom), vol. 55, no. 8, pp. 1948-1954, 2014.

[65] C. S. Chern, "Emulsion polymerization mechanisms and kinetics," Prog. Polym. Sci., vol. 31, no. 5, pp. 443-486, 2006.

[66] H. B. Yamak, "Emulsion Polymerization: Effects of Polymerization Variables on the Properties of Vinyl Acetate Based Emulsion Polymers," Emuls. Polym., pp. 3573, 2013.

[67] S. Minko, "Grafting on Solid Surfaces: "Grafting to" and "Grafting from" Methods," in Polymer Surfaces and Interfaces: Characterization, Modification and Applications, M. Stamm, Ed. Berlin, Heidelberg: Springer Berlin Heidelberg, 2008, 
pp. $215-234$.

[68] F. Barahona, E. Turiel, P. A. G. Cormack, and A. Martín-Esteban, "Chromatographic performance of molecularly imprinted polymers: Core-shell microspheres by precipitation polymerization and grafted MIP films via inifertermodified silica beads," J. Polym. Sci. Part A Polym. Chem., vol. 48, no. 5, pp. 10581066, 2010.

[69] K. Haupt, "Molecularly imprinted polymers: The next generation," Anal. Chem., vol. 75, pp. 376A-383A, 2003.

[70] and W. . Zhao.K.K, Zhang.W, Zhang. H.D, Du.H, Li.Q, Wang.S.Y, “A Molecular Imprinted Polymers with $\beta$-Cyclodextrin for Adsorption of Paeoniflorin," Asian J. Chem., vol. 27, no. 4, pp. 1435-1438, 2015.

[71] Y. K. Lv, C. X. Zhao, P. Li, Y. D. He, Z. R. Yang, and H. W. Sun, "Preparation of doxycycline-imprinted magnetic microspheres by inverse-emulsion suspension polymerization for magnetic dispersion extraction of tetracyclines from milk samples," J. Sep. Sci., vol. 36, no. 16, pp. 2656-2663, 2013.

[72] J. He et al., "Synthesis of fragment-imprinted microspheres of 2,6dichloropyrimidine as templates and determination of sulfonamides in milk samples," Chromatographia, vol. 76, no. 15-16, pp. 959-965, 2013.

[73] X. Su et al., "Synthesis and characterization of core-shell magnetic molecularly imprinted polymers for solid-phase extraction and determination of Rhodamine B in food," Food Chem., vol. 171, pp. 292-297, 2015. 
[74] Z. Yu, Q. Su, Y. Tang, and Z. Xu, "Preparation and evaluation of aconitine imprinted microspheres and its application to body fluid samples," J. Appl. Polym. Sci., vol. 128, no. 5, pp. 3425-3431, 2013.

[75] T. Kitabatake, H. Tabo, H. Matsunaga, and J. Haginaka, "Preparation of monodisperse curcumin-imprinted polymer by precipitation polymerization and its application for the extraction of curcuminoids from Curcuma longa L.," Anal. Bioanal. Chem., vol. 405, no. 20, pp. 6555-61, 2013.

[76] C. Zhao et al., "One-pot method for obtaining hydrophilic tetracycline-imprinted particles via precipitation polymerization in ethanol," J. Appl. Polym. Sci., vol. 131, no. 7, p. n/a-n/a, 2014.

[77] F. Puoci, S. Hampel, O. I. Parisi, A. Hassan, G. Cirillo, and N. Picci, "Imprinted microspheres doped with carbon nanotubes as novel electroresponsive drug-delivery systems," J. Appl. Polym. Sci., vol. 130, no. 2, pp. 829-834, 2013.

[78] H. Hang et al., "Selective separation of lambdacyhalothrin by porous/magnetic molecularly imprinted polymers prepared by Pickering emulsion polymerization,” $J$ Sep Sci, vol. 36, no. 19, pp. 3285-3294, 2013.

[79] X.-J. Li, S.-A. Zhong, and C.-E. Li, "Synthesis of novel photoresponsive molecularly imprinted polymer microspheres with special binding properties," $J$. Appl. Polym. Sci., vol. 130, no. 2, pp. 869-876, 2013.

[80] Y. J. Zhang, R. Feng, N. Qi, Y. P. Zhang, L. Y. Bai, and M. X. Huang, "Synthesis and evaluation of cyromazine molecularly imprinted polymeric microspheres by two-step seed swelling polymerization," Asian J. Chem., vol. 25, no. 15, pp. 8329- 
8332, 2013.

[81] J. Wolska and M. Bryjak, "Removal of Bisphenol A from Aqueous Solution by Molecularly Imprinted Polymers," Sep. Sci. Technol., vol. 49, no. 11, pp. $1643-$ 1653, 2014.

[82] M. Bougrini et al., "Development of a novel sensitive molecularly imprinted polymer sensor based on electropolymerization of a microporous-metal-organic framework for tetracycline detection in honey," Food Control, vol. 59, pp. 424-429, 2016.

[83] W. Chen et al., "Molecularly imprinted hollow sphere array for the sensing of proteins," J. Biophotonics, vol. 8, no. 10, pp. 838-845, 2015.

[84] W. Stöber, A. Fink, and E. Bohn, "Controlled growth of monodisperse silica spheres in the micron size range," J. Colloid Interface Sci., vol. 26, no. 1, pp. 62-69, 1968.

[85] N. Karaseva, T. Ermolaeva, and B. Mizaikoff, "Piezoelectric sensors using molecularly imprinted nanospheres for the detection of antibiotics," Sensors Actuators B Chem., vol. 225, pp. 199-208, 2016.

[86] B. He, T. J. Morrow, and C. D. Keating, "NIH Public Access," Challenges, vol. 12, no. 5 , pp. 522-528, 2009.

[87] S. Ge, C. Ma, H. Yang, Q. Kong, Z. Bian, and J. Yu, "One novel molecular imprinting nanowires chemiluminescence sensor: preparation and pendimethalin recognition," Monatshefte für Chemie - Chem. Mon., vol. 146, no. 3, pp. 493-499, 2015. 
[88] T. Díaz-Faes López, M. E. Díaz-García, and R. Badía-Laîno, "Molecularly imprinted silica-silver nanowires for tryptophan recognition," Nanotechnology, vol. 25, no. 42, pp. 1-10, 2014.

[89] T. Chen, M. Shao, H. Xu, S. Zhuo, S. Liu, and S.-T. Lee, "Molecularly imprinted polymer-coated silicon nanowires for protein specific recognition and fast separation," J. Mater. Chem., vol. 22, no. 1, pp. 3990-3996, 2012.

[90] R. I. Boysen, L. J. Schwarz, S. Li, J. Chowdhury, and M. T. W. Hearn, "Photolithographic patterning of biomimetic molecularly imprinted polymer thin films onto silicon wafers," Microsyst. Technol., vol. 20, pp. 2037-2043, 2014.

[91] S. N. Egli, E. D. Butler, and C. S. Bottaro, "Selective extraction of light polycyclic aromatic hydrocarbons in environmental water samples with pseudo-template thinfilm molecularly imprinted polymers," Anal. Methods, vol. 7, pp. 2028-2035, 2015.

[92] N. Arabzadeh, A. Khosravi, A. Mohammadi, and N. M. Mahmoodi, "Enhanced photodegradation of hazardous tartrazine by composite of nanomolecularly imprinted polymer-nanophotocatalyst with high efficiency," Desalin. Water Treat., vol. 57, no. ahead-of-print, pp. 1-10, 2014.

[93] W. Siripairoj, A. Kaewchada, and A. Jaree, "Synthesis of molecularly imprinted polymers for the separation of gamma-oryzanol by using methacrylic acid as functional monomer," J. Taiwan Inst. Chem. Eng., vol. 45, no. 2, pp. 338-346, 2014.

[94] I. Haq et al., "Developing imprinted polymer nanoparticles for the selective separation of antidiabetic drugs," J. Sep. Sci., vol. 38, no. 19, pp. 3469-3476, 2015. 
[95] S. Bajpai, SK; Jhariya, "Selective Removal of Amikacin From Simulated Polluted Water Using Molecularly Imprinting Polymer (MIP)," J. Macromol. Sci. PART APURE Appl. Chem., vol. 52, no. 11, pp. 901-911, 2015.

[96] R. J. Uzuriaga-Sanchez, S. Khan, A. Wong, G. Picasso, M. I. Pividori, and M. D. P. T. Sotomayor, "Magnetically separable polymer (Mag-MIP) for selective analysis of biotin in food samples," Food Chem., vol. 190, pp. 460-467, 2016.

[97] H. Liu et al., "A photoswitchable organocatalyst based on a catalyst-imprinted polymer containing azobenzene," RSC Adv., vol. 5, no. 77, pp. 62539-62542, 2015.

[98] W. Sun, R. Tan, W. Zheng, and D. Yin, "Molecularly imprinted polymer containing Fe(III) catalysts for specific substrate recognition," Chinese J. Catal., vol. 34, no. 8, pp. 1589-1598, 2013.

[99] J. Czulak, A. Jakubiak-Marcinkowska, and A. Trochimczuk, "Polymer catalysts imprinted with metal ions as biomimics of metalloenzymes," Adv. Mater. Sci. Eng., vol. 2013, pp. 1-9, 2013.

[100] P. Tang, J. Huo, H. Zhang, H. Lei, and X. Lin, "Surface Plasmon Resonance-based Inhibitive Immunoassay Coupled with Dummy Template Molecularly Imprinted Polymer Solid Phase Extraction for On-line Analysis of Trace Clenbuterol," J. Chinese Chem. Soc., vol. 61, no. 12, pp. 1357-1364, 2014.

[101] Z. Lian, X. He, and J. Wang, "Determination of sulfadiazine in Jiaozhou Bay using molecularly imprinted solid-phase extraction followed by high-performance liquid chromatography with a diode-array detector," J. Chromatogr. B Anal. Technol. Biomed. Life Sci., vol. 957, pp. 53-59, 2014. 
[102] M. M. Sanagi et al., "Molecularly imprinted polymer solid-phase extraction for the analysis of organophosphorus pesticides in fruit samples," J. Food Compos. Anal., vol. 32, no. 2, pp. 155-161, 2013.

[103] J. He et al., "Novel restricted access materials combined to molecularly imprinted polymers for selective solid-phase extraction of organophosphorus pesticides from honey," Food Chem., vol. 187, pp. 331-337, 2015.

[104] A. Sarafraz-Yazdi and N. Razavi, "Application of molecularly-imprinted polymers in solid-phase microextraction techniques," TrAC - Trends Anal. Chem., vol. 73, pp. 81-90, 2015.

[105] Y. Men, JY; Gao, BJ; Yao, L; Zhang, "Preparation and Characterization of Metronidazole-Surface Imprinted Microspheres MIP-PSSS/CPVA for ColonSpecific Drug Delivery System," J. Macromol. Sci. PART A-PURE Appl. Chem., vol. 51, no. 11, pp. 914-923, 2014.

[106] L. Tang et al., "Macromolecular crowding of molecular imprinting: A facile pathway to produce drug delivery devices for zero-order sustained release," Int. J. Pharm., vol. 496, no. 2, pp. 822-833, 2015.

[107] P. Wang et al., "Molecularly imprinted layer-coated hollow polysaccharide microcapsules toward gate-controlled release of water-soluble drugs," $R s c A d v$., vol. 4, no. 50, pp. 26063-26073, 2014.

[108] M. A. Abu-Dalo, N. S. Nassory, N. I. Abdulla, and I. R. Al-Mheidat, “Azithromycinmolecularly imprinted polymer based on PVC membrane for Azithromycin determination in drugs using coated graphite electrode," J. Electroanal. Chem., vol. 
751, pp. 75-79, 2015.

[109] T. A. Sergeyeva et al., "Colorimetric test-systems for creatinine detection based on composite molecularly imprinted polymer membranes," Anal. Chim. Acta, vol. 770, pp. 161-168, 2013.

[110] C. Gkementzoglou, O. Kotrotsiou, M. Koronaiou, and C. Kiparissides, "Development of a sandwich-type filtration unit packed with MIP nanoparticles for removal of atrazine from water sources," Chem. Eng. J., vol. 287, pp. 233-240, 2016.

[111] Y. Liu et al., "Selective separation of phenol from salicylic acid effluent over molecularly imprinted polystyrene nanospheres composite alumina membranes," Chem. Eng. J., vol. 286, pp. 622-631, 2016.

[112] N. A. Yusof, N. D. Zakaria, N. A. M. Maamor, A. H. Abdullah, and M. J. Haron, "Synthesis and characterization of molecularly imprinted polymer membrane for the removal of 2,4-dinitrophenol," Int. J. Mol. Sci., vol. 14, no. 2, pp. 3993-4004, 2013.

[113] C. Alvarez-lorenzo and C. Angel, Molecular Imprinting: A Historical Perspective. Handbook of Molecularly Imprinted Polymers, no. March. Shawbury, United Kingdom: A Smither Group company, 2013.

[114] C.-C. Liu, "Electrochemical Sensors," Biomed. Eng. HandbookSecond Ed., 2000.

[115] P. Jolly, V. Tamboli, R. L. Harniman, P. Estrela, C. J. Allender, and J. L. Bowen, "Aptamer-MIP hybrid receptor for highly sensitive electrochemical detection of prostate specific antigen,” Biosens. Bioelectron., vol. 75, pp. 188-195, 2016. 
[116] K. Graniczkowska, M. Pütz, F. M. Hauser, S. De Saeger, and N. V. Beloglazova, "Capacitive sensing of $\mathrm{N}$-formylamphetamine based on immobilized molecular imprinted polymers," Biosens. Bioelectron., vol. 92, no. September 2016, pp. 741$747,2017$.

[117] L. Chen, H. T. Lian, X. Y. Sun, and B. Liu, "Sensitive detection of L-5hydroxytryptophan based on molecularly imprinted polymers with graphene amplification,” Anal. Biochem., vol. 526, pp. 58-65, 2017.

[118] I. Bakas et al., "Electrochemical impedimetric sensor based on molecularly imprinted polymers/sol-gel chemistry for methidathion organophosphorous insecticide recognition.," Talanta, vol. 130, pp. 294-8, 2014.

[119] O. Gurtova, L. Ye, and F. Chmilenko, "Potentiometric propranolol-selective sensor based on molecularly imprinted polymer.," Anal. Bioanal. Chem., vol. 405, no. 1, pp. 287-295, 2013.

[120] H. Bagheri, A. Shirzadmehr, and M. Rezaei, "Designing and fabrication of new molecularly imprinted polymer-based potentiometric nano-graphene/ionic liquid/carbon paste electrode for the determination of losartan," J. Mol. Liq., vol. 212, pp. 96-102, 2015.

[121] T. S. Anirudhan and S. Alexander, "Design and fabrication of molecularly imprinted polymer-based potentiometric sensor from the surface modified multiwalled carbon nanotube for the determination of lindane ( $\gamma$-hexachlorocyclohexane), an organochlorine pesticide,” Biosens. Bioelectron., vol. 64, pp. 586-593, 2015.

[122] Y. Yang et al., "Glucose sensors based on electrodeposition of molecularly 
imprinted polymeric micelles: A novel strategy for MIP sensors," Biosens. Bioelectron., vol. 26, no. 5, pp. 2607-2612, 2011.

[123] Z. Wang, Y. Qin, C. Wang, L. Sun, X. Lu, and X. Lu, "Preparation of electrochemical sensor for lead(II) based on molecularly imprinted film," Appl. Surf. Sci., vol. 258, no. 6, pp. 2017-2021, 2012.

[124] S. Mamo and J. Gonzalez-Rodriguez, "Development of a Molecularly Imprinted Polymer-Based Sensor for the Electrochemical Determination of Triacetone Triperoxide (TATP)," Sensors, vol. 14, pp. 23269-23282, 2014.

[125] B. Liu, B. Xiao, L. Cui, and M. Wang, "Molecularly imprinted electrochemical sensor for the highly selective and sensitive determination of melamine," Mater. Sci. Eng. $C$, vol. 55, pp. 457-461, 2015.

[126] X. Tan et al., "Electrochemical sensor based on molecularly imprinted polymer reduced graphene oxide and gold nanoparticles modified electrode for detection of carbofuran," Sensors Actuators B Chem., vol. 220, pp. 216-221, 2015.

[127] L. Zhao, B. Zeng, and F. Zhao, "Electrochemical determination of tartrazine using a molecularly imprinted polymer - multiwalled carbon nanotubes - ionic liquid supported Pt nanoparticles composite film coated electrode," Electrochim. Acta, vol. 146, pp. 611-617, 2014.

[128] J. G. Pacheco, M. Castro, S. Machado, M. F. Barroso, H. P. A. Nouws, and C. Delerue-Matos, "Molecularly imprinted electrochemical sensor for ochratoxin A detection in food samples," Sensors Actuators B Chem., vol. 215, pp. 107-112, 2015. 
[129] A. Nezhadali and M. Mojarrab, "Fabrication of an electrochemical molecularly imprinted polymer triamterene sensor based on multivariate optimization using multi-walled carbon nanotubes," J. Electroanal. Chem., vol. 744, pp. 85-94, 2015.

[130] R. Lei, C. Guo, H. Xiong, C. Dong, X. Zhang, and S. Wang, "A novel electrochemical sensor for $\beta 2$-agonists with high sensitivity and selectivity based on surface molecularly imprinted sol-gel doped with antimony-doped tin oxide," Electroanalysis, vol. 26, no. 5, pp. 1004-1012, 2014.

[131] M. B. Gholivand and M. Torkashvand, "The fabrication of a new electrochemical sensor based on electropolymerization of nanocomposit gold nanoparticlesmolecularly imprinted polymer for determination of valganciclovir," Mater. Sci. Eng. C, vol. 59, pp. 594-603, 2015.

[132] G. Yang and F. Zhao, "Electrochemical sensor for dimetridazole based on novel gold nanoparticles@molecularly imprinted polymer," Sensors Actuators B Chem., vol. 220, pp. 1017-1022, Dec. 2015.

[133] Y. Li et al., "Supportless electrochemical sensor based on molecularly imprinted polymer modified nanoporous microrod for determination of dopamine at trace level," Biosens. Bioelectron., vol. 78, pp. 308-314, 2016.

[134] C. Xue et al., "Amperometric detection of dopamine in human serum by electrochemical sensor based on gold nanoparticles doped molecularly imprinted polymers.," Biosens. Bioelectron., vol. 49, pp. 199-203, 2013.

[135] A. Yarman and F. W. Scheller, "The first electrochemical MIP sensor for tamoxifen.," Sensors (Basel)., vol. 14, no. 5, pp. 7647-54, 2014. 
[136] Z. Wang, J. Li, X. Liu, J. Yang, and X. Lu, "Preparation of an amperometric sensor for norfloxacin based on molecularly imprinted grafting photopolymerization.," Anal. Bioanal. Chem., vol. 405, no. 8, pp. 2525-33, 2013.

[137] T. Alizadeh, M. Zare, M. R. Ganjali, P. Norouzi, and B. Tavana, “A new molecularly imprinted polymer (MIP)-based electrochemical sensor for monitoring 2,4,6trinitrotoluene (TNT) in natural waters and soil samples," Biosens. Bioelectron., vol. 25, no. 5, pp. 1166-1172, 2010.

[138] T. Alizadeh, M. R. Ganjali, P. Norouzi, M. Zare, and A. Zeraatkar, “A novel high selective and sensitive para-nitrophenol voltammetric sensor, based on a molecularly imprinted polymer-carbon paste electrode," Talanta, vol. 79, no. 5, pp. 1197-1203, 2009.

[139] J. de Ribamar Martins Neto, W. de Jesus Rodrigues Santos, P. R. Lima, S. M. C. N. Tanaka, A. A. Tanaka, and L. T. Kubota, "A hemin-based molecularly imprinted polymer (MIP) grafted onto a glassy carbon electrode as a selective sensor for 4aminophenol amperometric," Sensors Actuators B. Chem., vol. 152, no. 2, pp. 220 $225,2011$.

[140] J. Zhang, Y. Niu, S. Li, R. Luo, and C. Wang, "A molecularly imprinted electrochemical sensor based on sol-gel technology and multiwalled carbon nanotubes-Nafion functional layer for determination of 2-nonylphenol in environmental samples," Sensors Actuators, B Chem., vol. 193, pp. 844-850, 2014.

[141] Y. Zhao et al., "An electrochemical sensor for selective determination of sulfamethoxazole in surface water using a molecularly imprinted polymer modified 
BDD electrode," Anal. Methods, vol. 7, no. 6, pp. 2693-2698, 2015.

[142] S. Des Azevedo et al., "Molecularly imprinted polymer-hybrid electrochemical sensor for the detection of $\beta$-estradiol," Ind. Eng. Chem. Res., vol. 52, no. 39, pp. 13917-13923, 2013.

[143] D. Lakshmi et al., "Electrochemical Sensor for Catechol and Dopamine Based on a Catalytic Molecularly Imprinted Polymer-Conducting Polymer Hybrid Recognition Element," Anal. Chem., vol. 81, no. 9, pp. 3576-3584, 2009.

[144] I. Chianella et al., "Rational design of a polymer specific for microcystin-LR using a computational approach," Anal. Chem., vol. 74, no. 6, pp. 1288-1293, 2002.

[145] V. K. Gupta, M. L. Yola, T. Eren, and N. Atar, "Selective QCM sensor based on atrazine imprinted polymer: Its application to wastewater sample," Sensors Actuators B Chem., vol. 218, pp. 215-221, 2015.

[146] H. da Silva, J. Pacheco, J. Silva, S. Viswanathan, and C. Delerue-Matos, "Molecularly imprinted sensor for voltammetric detection of norfloxacin," Sensors Actuators B Chem., vol. 219, pp. 301-307, 2015.

[147] and R. M. T. Mohammed Rizk, Safaa Shafik Toubar, Hossam Ezz El-Din Sayour, Dalia Mohamed, "A new potentiometric sensor based on molecularly imprinted polymer for analysis of a veterinary drug imidocarb dipropionat," Eur. J. Chem., vol. 5, no. 1, pp. 18-24, 2014.

[148] L. Li, Y. Liang, and Y. Liu, "Designing of molecularly imprinted polymer-based potentiometric sensor for the determination of heparin.," Anal. Biochem., vol. 434, 
no. 2, pp. 242-6, 2013.

[149] O. Y. F. Henry, D. C. Cullen, and S. a. Piletsky, "Optical interrogation of molecularly imprinted polymers and development of MIP sensors: A review," Anal. Bioanal. Chem., vol. 382, pp. 947-956, 2005.

[150] P. Wang et al., "Photoelectrochemical Sensor Based on Molecularly Imprinted Polymer-Coated TiO2 Nanotubes for Lindane Specific Recognition and Detection,” J. Inorg. Organomet. Polym. Mater., vol. 23, no. 3, pp. 703-711, 2013.

[151] P. Wang, W. Dai, L. Ge, M. Yan, S. Ge, and J. Yu, "Visible light photoelectrochemical sensor based on Au nanoparticles and molecularly imprinted poly(o-phenylenediamine)-modified $\mathrm{TiO} 2$ nanotubes for specific and sensitive detection chlorpyrifos.," Analyst, vol. 138, no. 3, pp. 939-45, 2013.

[152] A. A. Bazrafshan, M. Ghaedi, Z. Rafiee, S. Hajati, and A. Ostovan, "Nano-sized molecularly imprinted polymer for selective ultrasound-assisted microextraction of pesticide Carbaryl from water samples: Spectrophotometric determination," $J$. Colloid Interface Sci., vol. 498, pp. 313-322, 2017.

[153] W. Zhang, X. W. He, Y. Chen, W. Y. Li, and Y. K. Zhang, "Composite of CdTe quantum dots and molecularly imprinted polymer as a sensing material for cytochrome c,” Biosens. Bioelectron., vol. 26, no. 5, pp. 2553-2558, 2011.

[154] L. Tan et al., "A fluorescent turn-on detection scheme for $\alpha$-fetoprotein using quantum dots placed in a boronate-modified molecularly imprinted polymer with high affinity for glycoproteins," Microchim. Acta, vol. 182, no. 15-16, pp. 2615$2622,2015$. 
[155] L. Xu, G. Fang, M. Pan, X. Wang, and S. Wang, "One-pot synthesis of carbon dotsembedded molecularly imprinted polymer for specific recognition of sterigmatocystin in grains,” Biosens. Bioelectron., vol. 77, pp. 950-956, 2016.

[156] X. Ren and L. Chen, "Preparation of molecularly imprinted polymer coated quantum dots to detect nicosulfuron in water samples," Anal. Bioanal. Chem., vol. 407, no. 26, pp. 8087-8095, 2015.

[157] Y. Xiong, Z. Ye, J. Xu, Y. Liu, and H. Zhang, "A microvolume molecularly imprinted polymer modified fiber-optic evanescent wave sensor for bisphenol A determination.," Anal. Bioanal. Chem., vol. 406, no. 9-10, pp. 2411-20, 2014.

[158] M. Mehrzad-Samarin, F. Faridbod, A. S. Dezfuli, and M. R. Ganjali, “A novel metronidazole fluorescent nanosensor based on graphene quantum dots embedded silica molecularly imprinted polymer," Biosens. Bioelectron., vol. 92, no. October 2016, pp. 618-623, 2017.

[159] M. Amjadi and R. Jalili, "Molecularly imprinted mesoporous silica embedded with carbon dots and semiconductor quantum dots as a ratiometric fluorescent sensor for diniconazole,” Biosens. Bioelectron., vol. 96, no. April, pp. 121-126, 2017.

[160] W. Liu et al., "A molecularly imprinted polymer based a lab-on-paper chemiluminescence device for the detection of dichlorvos.," Spectrochim. Acta. A. Mol. Biomol. Spectrosc., vol. 141, pp. 51-7, 2015.

[161] H. Duan, L. Li, X. Wang, Y. Wang, J. Li, and C. Luo, "A sensitive and selective chemiluminescence sensor for the determination of dopamine based on silanized magnetic graphene oxide-molecularly imprinted polymer," Spectrochim. Acta Part 
A Mol. Biomol. Spectrosc., vol. 139, pp. 374-379, 2015.

[162] S. Wang, L. Ge, L. Li, M. Yan, S. Ge, and J. Yu, "Molecularly imprinted polymer grafted paper-based multi-disk micro-disk plate for chemiluminescence detection of pesticide.," Biosens. Bioelectron., vol. 50, pp. 262-8, 2013.

[163] S. Ge, C. Zhang, F. Yu, M. Yan, and J. Yu, "Layer-by-layer self-assembly CdTe quantum dots and molecularly imprinted polymers modified chemiluminescence sensor for deltamethrin detection," Sensors Actuators, B Chem., vol. 156, no. 1, pp. 222-227, 2011.

[164] N. Sharma et al., "Molecularly Imprinted Polymer Waveguides for Direct Optical Detection of Low-Molecular-Weight Analytes," Macromol. Chem. Phys., vol. 215, no. 23, pp. 2295-2304, 2014.

[165] N. Cennamo, L. De Maria, G. D. Agostino, M. Pesavento, and L. Zeni, "Combined Molecularly Imprinted Polymer and Surface Plasmon Resonance transduction in Plastic Optical Fiber for monitoring oil- filled power transformers," Procedia Eng., vol. 87, pp. 532-535, 2014.

[166] N. Cennamo, G. D’Agostino, M. Pesavento, and L. Zeni, "High selectivity and sensitivity sensor based on MIP and SPR in tapered plastic optical fibers for the detection of 1-nicotine," Sensors Actuators, B Chem., vol. 191, pp. 529-536, 2014.

[167] N. Cennamo, L. De Maria, G. D’Agostino, L. Zeni, and M. Pesavento, “Monitoring of Low Levels of Furfural in Power Transformer Oil with a Sensor System Based on a POF-MIP Platform.," Sensors (Basel)., vol. 15, no. 4, pp. 8499-511, 2015. 
[168] A. M. Shrivastav, S. K. Mishra, and B. D. Gupta, "Surface Plasmon ResonanceBased Fiber Optic Sensor for the Detection of Ascorbic Acid Utilizing Molecularly Imprinted Polyaniline Film,” Plasmonics, vol. 10, pp. 1853-1861, 2015.

[169] A. M. Shrivastav, S. P. Usha, and B. D. Gupta, "Fiber optic profenofos sensor based on surface plasmon resonance technique and molecular imprinting," Biosens. Bioelectron., vol. 79, pp. 150-157, 2016.

[170] T. Kamra, T. Zhou, L. Montelius, J. Schnadt, and L. Ye, "Implementation of Molecularly Imprinted Polymer Beads for Surface Enhanced Raman Detection," Anal. Chem., vol. 87, p. 150430165942001, 2015.

[171] T. Kamra et al., "Photoconjugation of molecularly imprinted polymer nanoparticles for surface enhanced Raman detection of propranolol," ACS Appl. Mater. Interfaces, vol. 7, pp. 27479-27485, 2015.

[172] Y. Hu, S. Feng, F. Gao, E. C. Y. Li-chan, E. Grant, and X. Lu, "Detection of melamine in milk using molecularly imprinted polymers - surface enhanced Raman spectroscopy," FOOD Chem., vol. 176, pp. 123-129, 2015.

[173] X. Liu, D. Yu, Y. Yu, and S. Ji, "Preparation of a magnetic molecularly imprinted polymer for selective recognition of rhodamine B," Appl. Surf. Sci., vol. 320, pp. 138-145, 2014.

[174] C. Hsu et al., "Optical sensing of phenylalanine in urine via extraction with magnetic molecularly imprinted poly ( ethylene-co-vinyl alcohol ) nanoparticles," Nanotechnology, vol. 26, no. 30, p. 305502, 2015. 
[175] S. Chen, X. Li, Y. Guo, and J. Qi, “A Ag-molecularly imprinted polymer composite for efficient surface-enhanced Raman scattering activities under a low-energy laser.," Analyst, vol. 140, no. 9, pp. 3239-43, 2015.

[176] S. N. Chen, X. Li, S. Han, J. H. Liu, and Y. Y. Zhao, "Synthesis of surface-imprinted Ag nanoplates for detecting organic pollutants in water environments based on surface enhanced Raman scattering," RSC Adv., vol. 5, pp. 99914-99919, 2015.

[177] Y. Guo, L. Kang, S. Chen, and X. Li, "High performance surface-enhanced Raman scattering from molecular imprinting polymer capsulated silver spheres," Phys. Chem. Chem. Phys., vol. 17, no. Cv, pp. 21343-21347, 2015.

[178] L. Chang, Y. Ding, and X. Li, "Surface molecular imprinting onto silver microspheres for surface enhanced Raman scattering applications.," Biosens. Bioelectron., vol. 50, pp. 106-10, 2013.

[179] J. Ye, Y. Chen, and Z. Liu, “A Boronate Affinity Sandwich Assay: An Appealing Alternative to Immunoassays for the Determination of Glycoproteins," Angew. Chemie - Int. Ed., vol. 126, pp. 1-5, 2014.

[180] N. Casis, C. Busatto, M. M. Fidalgo de Cortalezzi, S. Ravaine, and D. a Estenoz, "Molecularly imprinted hydrogels from colloidal crystals for the detection of progesterone," Polym. Int., vol. 64, pp. 773-779, 2015.

[181] M. V. Foguel, X.-A. Ton, M. V. B. Zanoni, M. D. P. T. Sotomayor, K. Haupt, and B. Tse Sum Bui, "A molecularly imprinted polymer-based evanescent wave fiber optic sensor for the detection of basic red 9 dye," Sensors Actuators B Chem., vol. 218, pp. 222-228, 2015. 
[182] J. Xue, D. Li, L. Qu, and Y. Long, "Surface-imprinted core-shell Au nanoparticles for selective detection of bisphenol A based on surface-enhanced Raman scattering.," Anal. Chim. Acta, vol. 777, pp. 57-62, 2013.

[183] A. Guoliang, L. Zhuo, C. Xiaoying, F. Junyu, Z. Dahe, and F. Wei, "In-situ hydrothermal synthesis of molecularly imprinted polymers coated carbon dots for fluorescent detection of bisphenol A," Sensors Actuators B. Chem., vol. 228, pp. 302-307, 2016.

[184] H. Duan, L. Li, X. Wang, Y. Wang, J. Li, and C. Luo, “CdTe quantum dots@luminol as signal amplification system for chrysoidine with chemiluminescencechitosan/graphene oxide-magnetite-molecularly imprinting sensor," Spectrochim. Acta Part A Mol. Biomol. Spectrosc., vol. 153, pp. 535-541, 2016.

[185] Z. Guo, L. Chen, H. Lv, Z. Yu, and B. Zhao, "Magnetic imprinted surface enhanced Raman scattering (MI-SERS) based ultrasensitive detection of ciprofloxacin from a mixed sample," Anal. Methods, vol. 6, no. 6, pp. 1627-1632.

[186] S. Jiang et al., "Surface plasmon resonance sensor based on molecularly imprinted polymer film for detection of histamine," Sensors Actuators B. Chem., vol. 221, pp. $15-21,2015$.

[187] S. Li, Y. Ge, S. A. Piletsky, and J. Lunec, Molecularly Imprinted Sensors: Overview and Applications. Elsevier Science \& Technology, 2012.

[188] R. A. Meyers, Advances in molecular biology and medicine. Weinheim : WileyVCH Verlag GmbH \& Co. KGaA, [2015], 2015. 
[189] C. Liang, H. Peng, L. Nie, and S. Yao, "Bulk acoustic wave sensor for herbicide assay based on molecularly imprinted polymer," Fresenius. J. Anal. Chem., vol. 367, no. 6 , pp. $551-555,2000$.

[190] H. Peng, C. Liang, D. He, L. Nie, and S. Yao, "Bulk acoustic wave sensor using molecularly imprinted polymers as recognition elements for the determination of pyrimethamine.," Talanta, vol. 52, no. 3, pp. 441-8, 2000.

[191] H. Peng, C. Liang, A. Zhou, Y. Zhang, Q. Xie, and S. Yao, "Development of a new atropine sulfate bulk acoustic wave sensor based on a molecularly imprinted electrosynthesized copolymer of aniline with o-phenylenediamine," Anal. Chim. Acta, vol. 423, pp. 221-228, 2000.

[192] C. H. Luo, M. Q. Liu, Y. C. Mo, J. N. Qu, and Y. L. Feng, "Thickness-shear mode acoustic sensor for atrazine using molecularly imprinted polymer as recognition element," Anal. Chim. Acta, vol. 428, no. 1, pp. 143-148, 2001.

[193] C. Tancharoen and W. Sukjee, "Molecularly Imprinted Polymer for Explosive Detection," pp. 1-4, 2015.

[194] H. He et al., "Detection of trace microcystin-LR on a 20MHz QCM sensor coated with in situ self-assembled MIPs," Talanta, vol. 131, pp. 8-13, 2015.

[195] M. Hussain, H. Northoff, and F. K. Gehring, "Detection of HIT antibody dependent platelet aggregation using novel surface imprinting approach.," Talanta, vol. 147, pp. 1-7, 2016.

[196] A. Physics and E. Birlik, "Ligand exchange and MIP-based paraoxon memories onto 
QCM sensor Ligand exchange and MIP-based paraoxon memories," no. November, pp. 351-357, 2015.

[197] V. K. Gupta, M. L. Yola, and N. Atar, "A novel molecular imprinted nanosensor based quartz crystal microbalance for determination of kaempferol," Sensors Actuators, B Chem., vol. 194, pp. 79-85, 2014.

[198] A. Kumar Singh and M. Singh, "QCM sensing of melphalan via electropolymerized molecularly imprinted polythiophene films," Biosens. Bioelectron., vol. 74, pp. 711-717, 2015.

[199] F. Di Pietrantonio et al., "A Shear horizontal surface acoustic wave biosensor for a rapid and specific detection of d-serine," Sensors Actuators, B Chem., vol. 226, pp. $1-6,2016$.

[200] A. Tretjakov, V. Syritski, J. Reut, R. Boroznjak, and A. Öpik, "Molecularly imprinted polymer film interfaced with Surface Acoustic Wave technology as a sensing platform for label-free protein detection," Anal. Chim. Acta, vol. 902, pp. 182-188, 2016.

[201] N. Maouche et al., "A surface acoustic wave sensor functionalized with a polypyrrole molecularly imprinted polymer for selective dopamine detection," $J$. Mol. Recognit., vol. 28, no. 11, pp. 667-678, 2015.

[202] N. Ktari et al., "Surface acoustic wave sensor for selective detection of flumequine," Procedia Eng., vol. 120, pp. 998-1002, 2015.

[203] N. Ktari et al., "Design of a polypyrrole MIP-SAW sensor for selective detection of 
flumequine in aqueous media. Correlation between experimental results and DFT calculations," RSC Adv., vol. 5, no. 108, pp. 88666-88674, 2015.

[204] C. Umr, "SH-SAW Sensing System based on thin film molecularly imprinted polymer: Study of Volatile organic compounds adsorption,” 2013.

[205] Y. Chen et al., "Ultrasensitive Detection of Testosterone Using Microring Resonator with Molecularly Imprinted Polymers," Sensors, vol. 15, no. 12, pp. 31558-31565, 2015.

[206] M. J. Boyce, K. J. Baisley, E. V. Clark, and S. J. Warrington, “Are published normal ranges of serum testosterone too high? Results of a cross-sectional survey of serum testosterone and luteinizing hormone in healthy men,” BJU Int., vol. 94, no. 6, pp. 881-885, 2004.

[207] B. Tse Sum Bui, F. Merlier, and K. Haupt, "Toward the use of a molecularly imprinted polymer in doping analysis: Selective preconcentration and analysis of testosterone and epitestosterone in human urine," Anal. Chem., vol. 82, no. 11, pp. 4420-4427, 2010.

[208] I. Gavrilovic, K. Mitchell, A. D. Brailsford, D. A. Cowan, A. T. Kicman, and R. J. Ansell, "A molecularly imprinted receptor for separation of testosterone and epitestosterone, based on a steroidal cross-linker," Steroids, vol. 76, no. 5, pp. 478483, 2011.

[209] S. H. Lee, D. H. Lee, J. A. Lee, W. Y. Lee, B. C. Chung, and M. H. Choi, "Comparative GC-MS based in vitro assays of 5-reductase activity using rat liver S9 fraction," Mass Spectrometry Letters, vol. 3, no. 1. pp. 21-24, 2012. 
[210] B. Mathian et al., "Testosterone Measured by 10 Immunoassays and by IsotopeDilution Gas Chromatography - Mass Spectrometry in Sera from 116 Men, Women , and Children," Clin. Chem., vol. 49, no. 8, pp. 1381-1395, 2003.

[211] Y. Chen, M. Yazdanpanah, B. R. Hoffman, E. P. Diamandis, and P. Wong, "Rapid determination of serum testosterone by liquid chromatography-isotope dilution tandem mass spectrometry and a split sample comparison with three automated immunoassays," Clin. Biochem., vol. 42, no. 6, pp. 484-490, 2009.

[212] H. W. Vesper, J. C. Botelho, and Y. Wang, "Challenges and improvements in testosterone and estradiol testing," Asian J. Androl., vol. 16, no. August 2013, pp. 178-184, 2014.

[213] M. Peeters et al., "Thermal detection of histamine with a graphene oxide based molecularly imprinted polymer platform prepared by reversible additionfragmentation chain transfer polymerization," Sensors Actuators, B Chem., vol. 203, pp. 527-535, 2014.

[214] M. Yang et al., "Study on the molecularly imprinted polymers with methyltestosterone as the template," Talanta, vol. 81, no. 1-2, pp. 156-161, 2010.

[215] · J. R. canyová · R. G.-K. and K. R. ·Yaroslav B. · B. Buszewski, "Molecularly imprinted adsorbents for preconcentration and isolation of progesterone and testosterone by solid phase extraction combined with HPLC," Adsorption, vol. 16, no. 4-5, pp. 473-483, 2010.

[216] L. Qiu, W. Liu, M. Huang, and L. Zhang, "Preparation and application of solidphase microextraction fiber based on molecularly imprinted polymer for 
determination of anabolic steroids in complicated samples," J. Chromatogr. A, vol. 1217, no. 48, pp. 7461-7470, 2010.

[217] B. Tse Sum Bui and K. Haupt, "Preparation and evaluation of a molecularly imprinted polymer for the selective recognition of testosterone-application to molecularly imprinted sorbent assays," J. Mol. Recognit., vol. 24, no. 6, pp. 1123$1129,2011$.

[218] C. Zhou et al., "Molecularly imprinted photonic polymer as an optical sensor to detect chloramphenicol," Analyst, vol. 137, no. 19, p. 4469, 2012.

[219] P. Jiang, J. F. Bertone, K. S. Hwang, and V. L. Colvin, "Single-Crystal Colloidal Multilayers of Controlled Thickness," Chem. Mater., vol. 11, no. 8, pp. 2132-2140, 1999.

[220] J. RIddick, W. Bunger, and T. Sakano, Techniques of Chemistry: Organic Solvents., 4th editio., vol. Volume II. New York, NY: John Wiley and Sons, 1985.

[221] E. Serjeant, Ionisation Constants of Organic Acids in Aqueous Solution. New York, NY: Pergamon, 1979.

[222] R. W. Kibechu, M. A. Mamo, T. A. M. Msagati, S. Sampath, and B. B. Mamba, "Synthesis and application of reduced graphene oxide and molecularly imprinted polymers composite in chemo sensor for trichloroacetic acid detection in aqueous solution," Phys. Chem. Earth, vol. 76-78, pp. 49-53, 2014.

[223] A. Betatache, F. Lagarde, C. Sanglar, A. Bonhomme, D. Leonard, and N. JaffrezicRenault, "Gold Electrodes Modified with Molecular Imprinted Acrylate Polymer for 
Impedimetric Determination of Testosterone," Sensors \& Transducers, vol. 27, no. May, pp. 92-99, 2014.

[224] H. Inan et al., "Photonic crystals: emerging biosensors and their promise for pointof-care applications," Chem. Soc. Rev., vol. 46, no. 2, pp. 366-388, 2017.

[225] L.-Q. Wang, F.-Y. Lin, and L.-P. Yu, “A molecularly imprinted photonic polymer sensor with high selectivity for tetracyclines analysis in food," Analyst, vol. 137, no. 15, p. 3502, 2012.

[226] X. Wang, Z. Mub, R. Liu, Y. Pu, and L. Yin, "Molecular imprinted photonic crystal hydrogels for the rapid and label-free detection of imidacloprid," Food Chem., vol. 141, no. 4, pp. 3947-3953, 2013.

[227] Z. Wu, C. Tao, C. Lin, D. Shen, and G. Li, "Label-Free Colorimetric Detection of Trace Atrazine in Aqueous Solution by Using Molecularly Imprinted Photonic Polymers," Chem. - A Eur. J., vol. 14, no. 36, pp. 11358-11368, 2008.

[228] D. Xu et al., "Label-free detection and discrimination of poly-brominated diphenylethers using molecularly imprinted photonic cross-reactive sensor arrays," Chem. Commun., vol. 50, no. 91, pp. 14133-14136, 2014.

[229] L. Qiu, W. Liu, M. Huang, and L. Zhang, "Preparation and application of solidphase microextraction fiber based on molecularly imprinted polymer for determination of anabolic steroids in complicated samples," J. Chromatogr. A, vol. 1217, no. 48, pp. 7461-7470, 2010.

[230] B. R. A. Thakur, C. Williard, A. Rajasekaran, T. Technology, and D. Group, "Using 
GC-MS / MS for Superior Sensitivity , Specificity and Precision in Free Testosterone Analysis,” no. September, pp. 22-24, 2010.

[231] K. and et al Socratis, “The Influence of Physical Training on Blood Levels of Human Growth Hormone, Testosterone and Procollagen in Young Rowers," Int. J. Kinesiol. Sport. Sci., vol. 4, no. 3, Aug. 2016.

[232] C. R. Andersson, J. Bergquist, and E. Theodorsson, "Comparisons between commercial salivary testosterone enzyme-linked immunosorbent assay kits," Scand. J. Clin. Lab. Invest., vol. 77, no. 8, pp. 582-586, 2017.

[233] A. Gårevik, NinaRane, L. Björkhem-Bergman, and L. Ekström, "Effects of different doses of testosterone and blood lipids in healthy men," Subst. Abuse Rehabil., vol. 5, pp. 121-127, 2014.

[234] O. Grotzinger, A. D. Grotzinger, F. D. Mann, and M. W. Patterson, "Corrigendum : Hair and Salivary Testosterone, Hair Cortisol, and Externalizing Behaviors in Adolescents Hair and Salivary Testosterone, Hair Cortisol, and Externalizing Behaviors in Adolescents," Assoc. Psychol. Sci., vol. 29, pp. 688-699, 2018.

[235] R. Ronay, L. Van Der Meij, J. K. Oostrom, and T. V Pollet, "No Evidence for a Relationship Between Hair Testosterone Concentrations and 2D : 4D Ratio or Risk Taking," Front. Behav. Neurosscience, vol. 12, no. March, pp. 1-11, 2018.

[236] M. H. Choi, Y. S. Yoo, and B. C. Chung, "Measurement of testosterone and pregnenolone in nails using gas chromatography-mass spectrometry," $J$. Chromatogr. B, vol. 754, no. 2, pp. 495-501, 2001. 
[237] B. Jin and X. Wang, "Synthesis of magnetic molecularly imprinted polymers with excellent biocompatibility for the selective separation and inhibition of testosterone in prostate cancer cells,” pp. 2979-2993, 2017.

[238] A. Zulfiqar, G. Morgan, and N. W. Turner, "Detection of multiple steroidal compounds in synthetic urine using comprehensive gas chromatography-mass spectrometry (GC×GC-MS) combined with a molecularly imprinted polymer cleanup protocol," Analyst, vol. 139, no. 19, pp. 4955-4963, 2014.

[239] A. J. O. Whitehouse et al., "Sex-specific associations between umbilical cord blood testosterone levels and language delay in early childhood," J. Child Psychol. Pschiatry, vol. 53, no. 7, pp. 726-734, 2012.

[240] A. J. Kadhem, S. Xiang, S. Nagel, C. H. Lin, and M. F. de Cortalezzi, "Photonic molecularly imprinted polymer film for the detection of testosterone in aqueous samples," Polymers (Basel)., vol. 10, no. 4, 2018.

[241] Z. Adali-Kaya, B. Tse Sum Bui, A. Falcimaigne-Cordin, and K. Haupt, "Molecularly imprinted polymer nanomaterials and nanocomposites: Atom-transfer radical polymerization with acidic monomers," Angew. Chemie - Int. Ed., vol. 54, no. 17, pp. 5192-5195, 2015.

[242] S. Ambrosini, S. Shinde, E. De Lorenzi, and B. Sellergren, "Glucuronide directed molecularly imprinted solid-phase extraction: isolation of testosterone glucuronide from its parent drug in urine," Analyst, vol. 137, no. 1, p. 249, 2012.

[243] H. Inan et al., "Photonic crystals: emerging biosensors and their promise for pointof-care applications," Chem. Soc. Rev., vol. 46, no. 2, pp. 366-388, 2017. 
[244] V. Krishnakumar and V. Balachandran, "Analysis of vibrational spectra of 5-fluoro, 5-chloro and 5-bromo-cytosines based on density functional theory calculations," Spectrochim. Acta - Part A Mol. Biomol. Spectrosc., vol. 61, no. 5, pp. 1001-1006, 2005.

[245] L. M. Azofra, I. Alkorta, and J. Elguero, "Theoretical study of the mutarotation of erythrose and threose: Acid catalysis," Carbohydr. Res., vol. 372, pp. 1-8, 2013.

[246] D. Z. Lin, H., Wu, D. L., Liu, L., \& Jia, “Theoretical study on molecular structures, intramolecular proton transfer reaction, and solvent effects of 1-phenyl-3-methyl-4(6-hydro-4-amino-5-sulfo-2, 3-pyrazine)-pyrazole-5-one,” J. Mol. $\quad$ Struct. THEOCHEM, vol. 850(1-3), pp. 32-37, 2008.

[247] P. Kolar, J. Classen, and S. G. Hall, "Physicochemical data of p-cresol, butyric acid, and ammonia," Data Br., vol. 26, p. 104356, 2019.

[248] Y. J. Huang, R. Chang, and Q. J. Zhu, "Synthesis and characterization of a Molecularly imprinted polymer of spermidine and the exploration of its molecular recognition properties," Polymers (Basel)., vol. 10, no. 12, pp. 1-17, 2018.

[249] M. C. Labritories, “Test Definition: TTFB, Testosterone, Total, Bio, Free, S,” 2020.

[250] M. C. Labritories, “Test Definition: DHTS, Dihydrotestosterone, S,” 2020.

[251] M. C. Labritories, “Test Definition: DHEA, Dehydroepiandrosterone, S,” 2020.

[252] M. C. Labritories, “Test Definition: SALCT, Cortisol, Saliva,” 2020.

[253] M. C. Labritories, “Test Definition: EEST, Estradiol, Mass Spectrometry, S,” 2020.

[254] M. C. Labritories, “Test Definition: ANST, Androstenedione, S,” 2020. 
[255] Healthline, "Hair Loss and Testosterone," 2020. .

[256] S. N. Eberhard Nieschlag, Hermann M. Behre, Testosterone: Action, Deficiency, Substitution, 4 th edith. New York: Cambridge University Press, 2012.

[257] S. Rinaldi et al., "Validity of free testosterone and free estradiol determinations in serum samples from postmenopausal women by theoretical calculations," Cancer Epidemiol. Biomarkers Prev., vol. 11, no. 10, pp. 1065-1071, 2002.

[258] M. C. Labritories, "Test Definition: DHEAS, Dehydroepiandrosterone Sulfate, S," 2020.

[259] W. Boufas et al., "Synthesis and antibacterial activity of sulfonamides. SAR and DFT studies," J. Mol. Struct., vol. 1074, pp. 180-185, 2014.

[260] F. Wang, W. Sun, W. Pan, and N. Xu, “Adsorption of sulfamethoxazole and 17ßestradiol by carbon nanotubes/CoFe2O4composites," Chem. Eng. J., vol. 274, pp. $17-29,2015$.

[261] National institue of Environmental Health Science, "Endocrine Disruptors." [Online].

Available: https://www.niehs.nih.gov/health/topics/agents/endocrine/index.cfm.

[262] H. S. Chang, K. H. Choo, B. Lee, and S. J. Choi, "The methods of identification, analysis, and removal of endocrine disrupting compounds (EDCs) in water," $J$. Hazard. Mater., vol. 172, no. 1, pp. 1-12, 2009.

[263] N. H. Tran, J. Hu, and S. L. Ong, "Simultaneous determination of PPCPs, EDCs, and artificial sweeteners in environmental water samples using a single-step SPE 
coupled with HPLC-MS/MS and isotope dilution," Talanta, vol. 113, pp. 82-92, 2013.

[264] R. Ben Sghaier et al., "A Combination of Factorial Design, Off-line SPE and GCMS Method for Quantifying Seven Endocrine Disrupting Compounds in Water,” Int. J. Environ. Res., vol. 11, no. 5-6, pp. 613-624, 2017.

[265] E. Tatay and S. Esp, "Estrogenic activity of zearalenone, a -zearalenol and b zearalenol assessed using the E-screen assay in MCF-7 cells," vol. 28, no. 4, pp. 239-242, 2018.

[266] W. Guo, K. Van Langenhove, T. Vandermarken, M. S. Denison, and M. Elskens, "In situ measurement of estrogenic activity in various aquatic systems using organic di ff usive gradients in thin- fi $1 \mathrm{~m}$ coupled with ERE-CALUX bioassay," Environ. Int., vol. 127, no. February, pp. 13-20, 2019.

[267] T. M. Edwards, H. E. Morgan, C. Balasca, N. K. Chalasani, L. Yam, and A. M. Roark, "Detecting Estrogenic Ligands in Personal Care Products using a Yeast Estrogen Screen Optimized for the Undergraduate Teaching Laboratory," no. January, pp. 1-11, 2018.

[268] X. Lv et al., "Multicolor and Ultrasensitive Enzyme-Linked Immunosorbent Assay Based on the Fluorescence Hybrid Chain Reaction for Simultaneous Detection of Pathogens," 2019.

[269] C. Teng et al., "Bisphenol A affects androgen receptor function via multiple mechanisms," Chem. Biol. Interact., vol. 203, no. 3, pp. 556-564, 2013. 
[270] G. A. M. Kahl, J. Cavallin, E. Durhan, K. Jensen, E. Makynen, D Martinovic, L. Wehmas, D. Villeneuve, "A Time-Course Study with the Androgen Receptor Antagonist Flutamide in Fish," U.S. Environ. Prot. Agency, Mid-Continent Ecol. Div. Duluth MN, pp. 869-876, 2011.

[271] D. L. S. and D. P. S. Stephen A. Martin, Jr., Siobán D. Harlow, Mary Fran Sowers, Matthew P. Longnecker, David Garabrant, "DDT Metabolite and Androgens in African-American Farmers,” Epidemiology, vol. 13, no. 4, pp. 454-458, 2002.

[272] A. Matsushima, "A novel action of endocrine-disrupting chemicals on wildlife; ddt and its derivatives have remained in the environment," Int. J. Mol. Sci., vol. 19, no. $5,2018$.

[273] J. Emily Monosson, William R. Kelce, Christy Lambright, Joseph Ostby, L. Earl Gray, "Peripubertal exposure to the antiandrogenic fungicide, vinclozolin, delays puberty, inhibits the development of androgen-dependent tissues, and alters androgen receptor function in the male rat," Toxicol. Ind. Health, vol. 15, no. 1-2, 1999.

[274] G. Viswanath, S. Chatterjee, S. Dabral, S. R. Nanguneri, G. Divya, and P. Roy, “Anti-androgenic endocrine disrupting activities of chlorpyrifos and piperophos," $J$. Steroid Biochem. Mol. Biol., vol. 120, no. 1, pp. 22-29, 2010.

[275] N. C. for A. transaltional S. US department of health and Human Services, "Drugs," DICHLOROPHEN, 2020. .

[276] T. U. of N. C. at C. H. G. S. of G. P. Health and Version, "TOXICOLOGICAL PRIORITY INDEX GUI User.” pp. 1-22. 
[277] U.S. Environmental Protection Agency, "ECOTOX User Guide: ECOTOXicology Knowledgebase System. Version 5.0,” 2020. .

[278] E. United States Environmental Protection Agenc, "Toxicity Forecasting, Advancing the Next Generation of Chemical Evaluation," 2020. [Online]. Available: https://www.epa.gov/chemical-research/toxicity-forecasting.

[279] J. A. Wignall et al., "Conditional toxicity value (CTV) predictor: An in silico approach for generating quantitative risk estimates for chemicals," Environ. Health Perspect., vol. 126, no. 5, pp. 1-13, 2018.

[280] H. Fang et al., "Study of 202 Natural, Synthetic, and Environmental Chemicals for Binding to the Androgen Receptor," Chem. Res. Toxicol., vol. 16, no. 10, pp. 13381358, 2003. 
Abbas Kadhem was born in Baghdad, Iraq on Christmas eve. After completing his high school, he received his degree in Bachelor of Science majoring in Environmental Engineering at Al- mustansiriya University, Baghdad, Iraq in 2001. Afterward, Abbas had worked with one of the famous Environmental non-government organizations (Nature Iraq) which included scientists from the US, the Italian Republic, and the Republic of Iraq. This organization worked in several projects for the restoration of Iraqi marshes and other environmental protection projects. At the same time, he completed his master's degree in Environmental Engineering at the same university in 2005. His ambition did not stop there, he got a faculty job at the University of Technology, Baghdad, Iraq which gave him a great opportunity to work at the staff of the environmental research center of that university. Abbas attended the University of Missouri for the Ph.D. program in January 2015 in the Civil and Environmental Engineering Department. During his Ph.D. study, he was focused on the detection of contaminants in water. He proposed a novel sensor to detect the testosterone hormone in aqueous samples by using molecularly imprinted polymer. 